\title{
Severe muscle atrophy due to spinal cord injury can be reversed in complete absence of peripheral nerves
}

\author{
Simona Boncompagni \\ Department of Physiology, Center of Research on Aging. (Ce.S.I), Gabriele \\ d'Annunzio University, Chieti, Italy
}

\begin{abstract}
In the last years, a new efficient treatment has been developed to treat paralyzed skeletal muscle of patients affected by spinal cord injury (SCI). The capability of the functional electrical stimulation (FES) to improve trophism and in some cases muscle function, are now well documented both in animals after experimental cord lesion, and in humans, generally after traumatic cord lesion. This new findings makes FES an important tool for the rehabilitation of SCI patients. FES stimulation has been proven to be an effective method used to retard muscle atrophy and improve recovery after reinnervation. Sophisticated FES devices have been developed for restoring function in the upper and lower extremities, the bladder and bowel, and the respiratory system of SCI patients. However, there are SCI cases, such as those affected by flaccid paralysis, in which the musculature is not treated with FES rehabilitation therapy. This is because conventional FES apparatuses are designed for direct stimulation of peripheral nerves that need small currents to be depolarized, and are not effective in patients that have lost their peripheral nerves, and, therefore, require higher currents for the direct depolarization of the muscle fibers. Lack of muscle treatment generates, as a secondary problem, a long series of alterations to tissues other than muscle, such as bones (osteoporosis), skin (pressure sores, decubital ulcers), etc., that are a direct consequence of inactivity and poor blood supply to the denervated areas. These complications represent an extremely serious problem for the general health of the injured individuals, who usually have a shorter than normal life span. In the hopes of changing this common belief, an innovative rehabilitation procedure, based on FES, has been developed with the aim of reversing long-lasting muscle atrophy in the muscles of the lower extremities of SCI patients affected by complete lesion of the conus cauda, i.e. that have no peripheral nerves. Experimental and clinical results have shown that electrical stimulation training by long impulses can restore muscle mass, force production and movements even after long lasting complete denervation. Measurements by CT-scans revealed a substantial increase of tight muscle cross sectional area during the first years of FES and muscle function of the lower extremities was restored in some patients sufficiently to allow for supported standing, standing, and even for a few steps to be taken. We have described the ultrastructural changes accompanying the recovery of skeletal muscle in the total absence of either sensory or motor innervation. The results showed a striking structural recovery of muscle fiber ultrastructure in all FES treated patients: the $90 \%$ (or more) of the studied fibers recovery from a very profound atrophy under the influence of the electrical stimulation. Restoration of ultrastructure involves all the major apparatuses of muscle fibers, such as the one deputed to muscle activation and $\mathrm{Ca} 2+$ handling (ECC apparatus), to contractility (myofibrils), and to metabolic and energy generation tasks (mitochondria). This structural recovery occurs in complete absence of nerve endings, under the influence of muscle activity, and follows pattern that mimics in many aspects normal muscle differentiation as well as recovery after short-term disuse and/or denervation. The present ultra-structural studies are important because they show that, despite the apparent complete loss of specific structure, the long-term denervated fibers maintain their full differentiation program. Reversal of the damages from long-standing denervation in humans may be of significant importance also for the rehabilitation and the general health of SCI patients.
\end{abstract}

Key Words: Severe muscle atrophy, spinal cord injury denervation, FES

European Journal Translational Myology - Basic Applied Myology 2012; 22 (4): 161-200 
Muscle atrophy due to SCI can be reversed in complete absence of peripheral nerves

European Journal Translational Myology - Basic Applied Myology 2012; 22 (4): 161-200

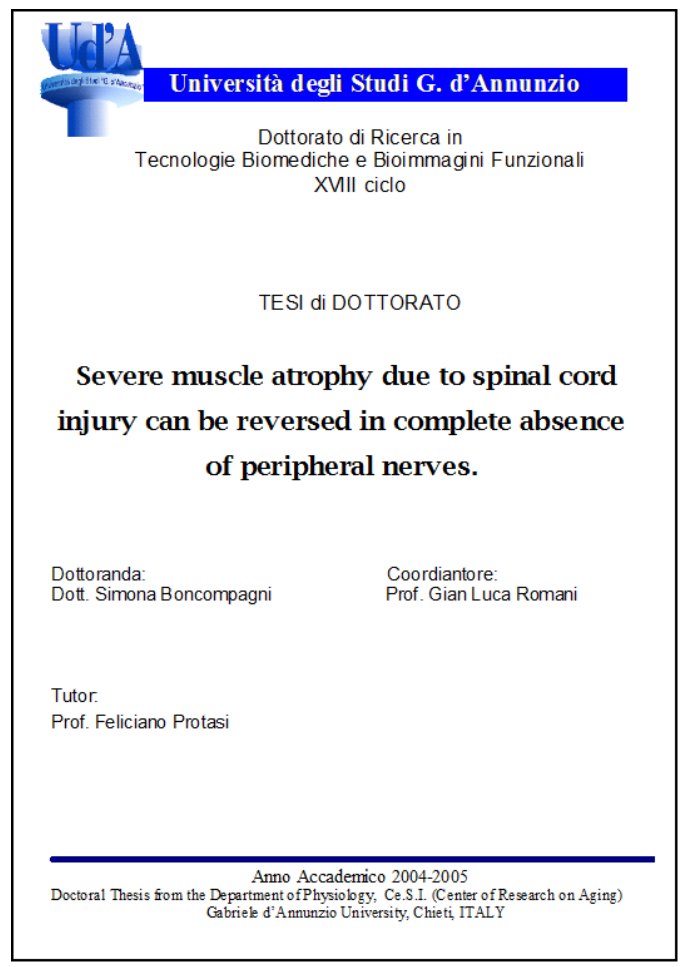

\section{INTRODUCTION}

1.1 SKELETAL MUSCLE

1.1.1 Muscle structure and function

1.1.2 The sarcoplasmic reticulum and the transverse tubules

1.1.3 Muscle Contraction and Excitation-Contraction Coupling (ECC)

1.1.4 Ultrastructure of Calcium Realise Units

1.1.5 RyRs and DHPRs: the two major players of ECC

1.1.6 Nerve and Neuromuscular Junctions

1.1.7 Trophic Interaction between Muscle and Nerve

1.2 SPINAL CORD INJURY

1.2.1 What is the spinal cord and the vertebra?

1.2.2 The Spinal Cord Injury

1.3 EFFECTS OF DENERVATION ON SKELETAL MUSCLE

1.4 FUNCTIONAL ELECTRICAL STIMULATION

1.4.1 Principles of FES Functional Electrical Stimulation

1.4.2 FES on patients with upper motoneurons lesion (spastic patients)

1.4.3 FES on patients with lower motoneuron lesion (completely denervated patients)

1.5 SCIENTIFIC MEANING OF THE PRESENT STUDY

2. MATERIALS AND METHODS

2.1 PATIENTS' CHARACTERISTICS

2.2 CLINICAL TESTING OF PATIENTS

2.2.1 Determination of muscle cross-sectional area of thigh muscles by CT scan

2.2.2 Force Measurements
2.3 MUSCLE BIOPSY

$2,3.1$ Needle muscle biopsy

2.3.2 Biopsy Specimens

2.4 ELECTRICAL STIMULATION TRAINING

2.4.1 Rehabilitation Training: stimulation parameters and protocols

2.4.2 Daily Therapy and Training Time

2.5 LIGHT MICROSCOPY

2.5.1 Hystology

2.6 ELECTRON MICROSCOPY

2.6.1 Classification of muscle fibers

2.6.2 Size distribution spectrum of total myofibers

2.6.3 Morphometric analysis

2.7 PREPARATION OF FIGURES

3. RESULTS

3.1 CLINICAL OBSERVATIONS

3.1.1 Force measurements

3.1.2 CT-cross sectional area and muscle density

3.2 LIGHT AND ELECTRON MICROSCOPY: LONG-TERM DENERVATED MUSCLE

3.2.1 Atrophy and dystrophy of human long-term denervated muscle

3.2.2 Severely atrophic myofibers: nuclear clumping and disorganization of the myofibrillar components

3.2.3 Myofibers regeneration in Long-Term Denervated Muscle

3.2.4 Ultrastructural analysis of long-term denervated muscle fibers

3.3 LIGHT AND ELECTRON MICROSCOPY: ELECTROSTIMULATED MUSCLE

3.3.1 Effects of the FES Training on Long-Term Denerveted muscle

3.3.2 Ultra-structural analysis of FES trained denervated fibers

4. DISCUSSION

4.1 LONG-TERM DENERVATED HUMAN MUSCLE

4.2 CLINICAL IMPORTANCE OF FES TRAINING IN SCI PATIENTS

4.3 IMPORTANCE OF FES ON DENERVATED SKELETAL MUSCLE

\section{INTRODUCTION}

\subsection{SKELETAL MUSCLE}

1.1.1 Muscle structure and function

During the past century, extensive investigations have revealed the general structure and function of skeletal muscle. Though much is known about the structural organization of muscle, the constitutive equations describing its behavior have yet to be derived. In this section emphasis is placed on describing the structural organization of skeletal muscle. The description begins at the level of the gross whole muscle and proceeds to the smaller subunits, concluding with the proteins making up the myofilaments. The description given here is quite detailed since muscle behavior is directly 


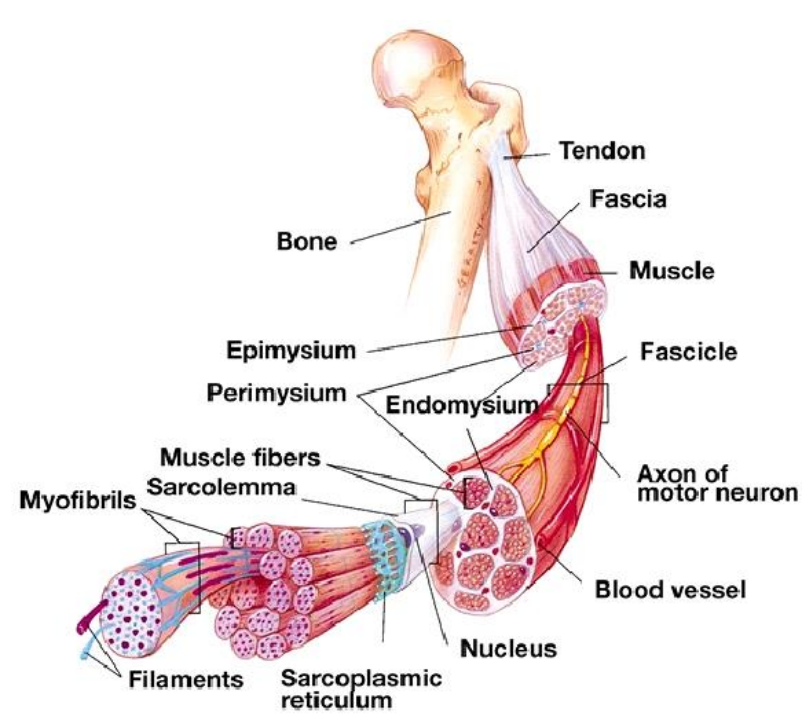

Fig 1 Schematic representation of the structural hierarchy of skeletal muscle from muscle to myofibrils. The gross muscle is composed of bundles of fascicles that consist on groups of fibers. Fibers can be further divided into myofibrils that contain the myofilaments making up the sarcomeres.

related to its structure. Skeletal muscle, as its name implies, is the muscle attached to the skeleton by tendons (Figure 1). Clearly the major function of skeletal muscle is the generation of force especially for movement as well as holding bodies in position but also act upon the viscera to produce movement of organs, blood vessels and glands. Skeletal muscles have an abundant supply of blood vessels and nerves that is directly related to the outstanding property of the muscle: the contraction.

Muscle is composed of many subunits and complex structural arrangements (Figure 1). Groups of muscle fibers are surrounded by a connective tissue sheath known as perimysium (literally, "around muscle") and arranged in bundles called fascicles. These fascicles are also bundled together, surrounded by more connective tissue (epimysium, literally, "on top of muscle") to form the whole muscle, which we can inspect visually. The muscle fiber is completely enclosed by the plasma membrane which is usually referred to as the sarcolemma. The sarcolemma may be resolved into three layers, the plasmalemma, basal lamina, and a thin layer of collagenous fibrils [1,2]. Mononucleated satellite cells can be found between the basal lamina and the plasmalemma. These cells may be involved in forming new fibers following muscle trauma [2,3]. The largest functional unit of contractile filaments is the myofibril [4] (literally, "muscle thread") (Figure 2).

Myofibrillar diameter is about 1 to $3 \mu \mathrm{m}$, thus thousands of myofibrils can be packed into a single

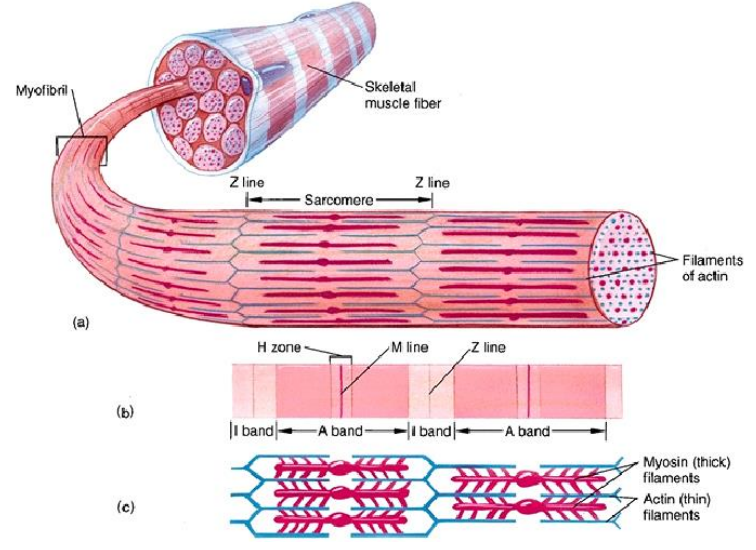

Fig 2 Organization of Contractile Proteins in Muscle. Each myofibril is composed of bundles of filamentous contractile proteins (actin and myosin), some extending from end to end in the cell. A single myofibril is composed of many short structural units, known as sarcomeres, which are arranged end to end. The proteins at the junctions between sarcomeres form the $Z$ line, and thus a sarcomere extends along a myofibril from one $Z$ line to the next $Z$ line.

muscle fiber: typically 10 to $100 \mu \mathrm{m}$ diameter and several centimeters long. Myofibrils, which constitute 75 - 85 percent of the fiber volume1, are arranged in parallel (side by side) to make up the muscle fiber. Myofibrils are subdivided into their component units known as sarcomeres, the functional unit of muscle contraction. A myofibril is therefore a number of sarcomeres (literally, "muscle segment") arranged in series. The total number of sarcomeres within a fiber depends on the muscle fiber length and diameter. Because of the series arrangement of sarcomeres within a myofibril, the total distance of myofibrillar shortening is equal to the sum of the individual shortening distances of the individual sarcomeres (Figure 3). This is why a whole muscle may shorten several centimeters even though each sarcomere can only shorten about $1 \mu \mathrm{m}$. It should also be stated that the number of sarcomeres in a mature muscle can change given the appropriate stimulus. This gives skeletal muscle a tremendous ability to adapt.

The terminology used to describe sarcomere anatomy is largely the result of muscle observations using polarizing microscopes (Figure 4).

When viewed with an electron microscope, specific zones of a muscle fiber appear darker than other zones. The dark zones have dense protein bands and stain deeply with basic dyes causing the plane of polarization of light to be rotated strongly. These zones have been labeled A-bands (for anisotropic). Other zones are less protein dense, stain weakly and rotate the plane of polarization of light weakly. These zones 
Muscle atrophy due to SCI can be reversed in complete absence of peripheral nerves

European Journal Translational Myology - Basic Applied Myology 2012; 22 (4): 161-200

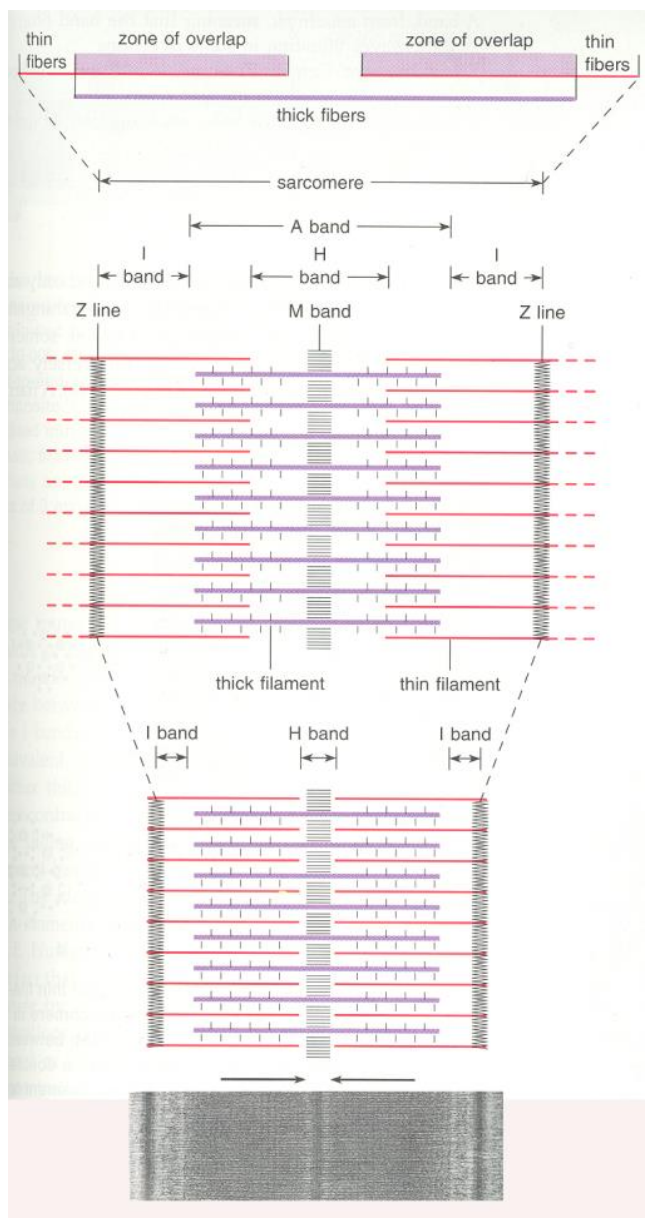

Fig 3 The illustrationn shows the structure of the bands in terms of the major proteins, actin \& myosin: the I band contains only the actin protein. Thin filaments: actin, attached to $Z$ line, found in both $A$ and I bands. Thick filaments: myosin, found in $A$ band in the $A$ band the 2 proteins overlap. When muscle contracts the actin filaments slide into the $A$ band, overlapping with myosin, the sarcomere shortens and the $Z$ lines move closer together

have been labeled I-bands (for isotropic) [5]. In the middle of the I-band is a dense protein zone called the Z-line or Z-disk (for Zwischen-Scheibe meaning interim disk) [6]. In the middle of the A-band is a dense protein zone called the H-zone (for HelleScheibe) [4,5]. In the center of the H-zone is a region called the M-line (for middle). The A-band corresponds to the zone of thick filaments. The H-zone is that region of the thick filaments that is not overlapped by the thin filaments. The M-line is composed of a connective tissue network binding the thick filaments and maintaining them in a hexagonal pattern when viewed in a transverse plane. The Z-disk is composed of a connective tissue network binding the thin filaments [7]. Thin filaments are attached at the Z-

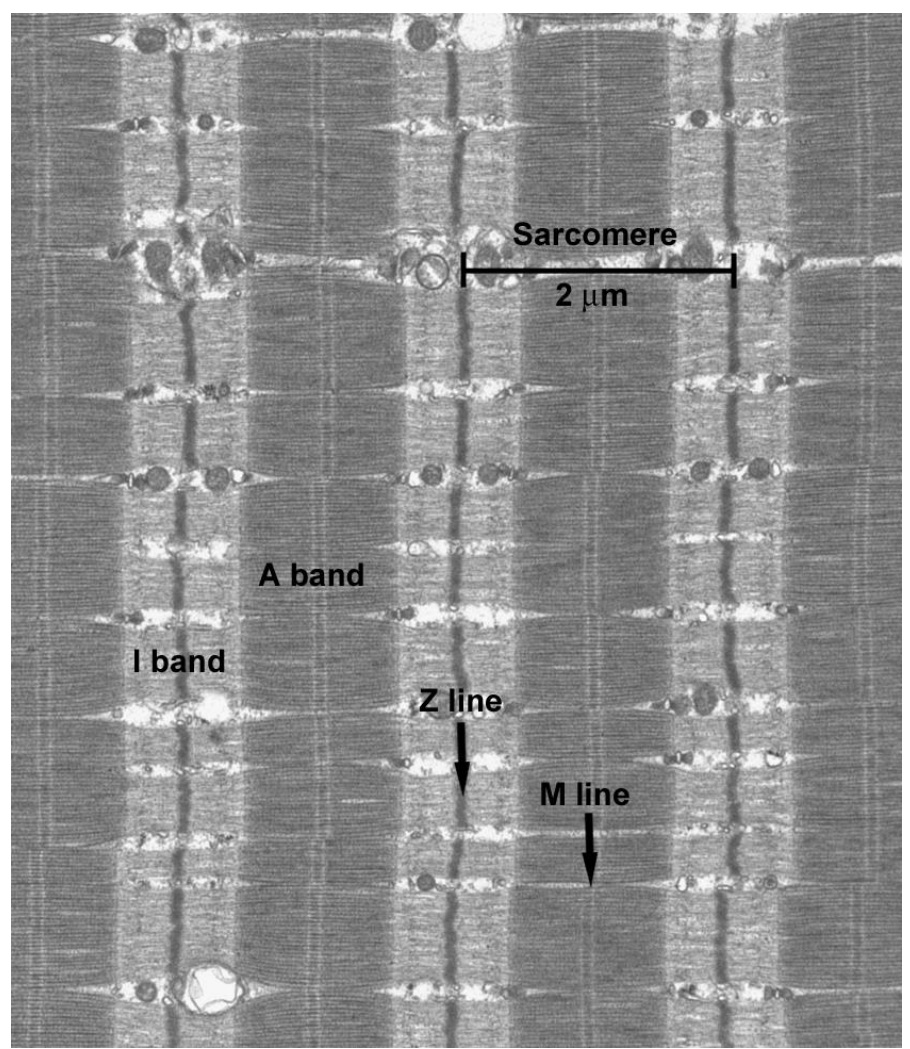

Fig 4 Electron microscopy picture of human skeletal muscle. The repeating units responsible for the striated appearance of myofibrils and muscle cells are clearly visible in the electron microscope. Each unit, about 2 um long in resting muscle, is called sarcomere. The boundaries of a sarcomere are marked on each end by a disc, called the $Z$ disc or $Z$ line. Each sarcomere contains an anisotropic (doubly refractive, therefore dark at the microscopy) band bounded by two isotropic (singly refractive, therefore light) bands. The anisotropic band is called the A band; the isotropic band is called the I band

disk but are free to interdigitate with the thick filaments at their other end. Myosin filaments are separated by $40-50 \mathrm{~nm} 1$ while the myosin/actin spacing is $20-30 \mathrm{~nm} 1$. A sarcomere is defined as the region between Z-disks in a myofibril. As a muscle shortens the sarcomere I-band and H-zone decrease in length while the A-band length remains constant (Figure 3). These observations lead to development of the sliding filament theory which is discussed in the section on muscle contraction $[8,9]$.

Clearly shown in the electron microscopy pictures (Figure 5 and Figure 6, are other important organelles of the skeletal muscle: structures called mitochondria. The principal function of these organelles is to produce the energy needed to power muscular contraction. 
Muscle atrophy due to SCI can be reversed in complete absence of peripheral nerves

European Journal Translational Myology - Basic Applied Myology 2012; 22 (4): 161-200
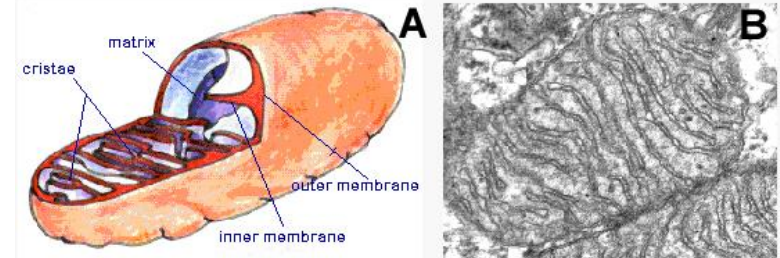

Fig 5 Mitochondria: the cells' power sources. (A) Schematic rappresentation of mitochondria. They are distinct organelles with two membranes. Usually they are rod-shaped, however they can be round. The outer membrane limits the organelle. The inner membrane is thrown into folds or shelves that project inward. These are called "cristae mitochondriales". (B) An electron micrograph from of an FDB of mouse, shows the organization of the two membranes.

Mitochondria supply energy for contraction through their oxidative metabolism. The number of mitochondria present within a cell reflects the metabolic pattern of the fiber. Fibers relying on oxidative metabolism have a greater number of mitochondria compared to fibers relying on anaerobic metabolism. In adult skeletal muscle, mitochondria are strategically located next to the muscle filaments and precisely targeted next to calcium release units (CRUs), the structures needed for the muscle contraction (see below for more details on muscle contraction), between the sarcomere A-I junction and the $\mathrm{Z}$ line (black arrowheads in Figure 6). In this way the energy produced in the mitochondria is readily transported to its site of use in the muscle filaments.

\subsubsection{The sarcoplasmic reticulum and the transverse tubules}

The muscle fiber contains two distinct membranous systems between the myofibrils (Figure 7); the transverse ( $\mathrm{T}$ ) tubular system that is part of the plasmalemma and makes a network of invaginations into the cell1 [10] and the sarcoplasmic reticulum (SR) $[1,11]$. In addition to the orderly array of muscle fibrils, the two intracellular membrane systems of skeletal muscle fibers, the SR and the T tubule system [12], also form an organized structure. The membrane of the SR is well organized system of tubules and vesicles, an highly specialized version of the endoplasmic reticulum of other cells, that closely surrounds myofibrils, running along the longitudinal axis of myofibrils. The T-tubule system provides a structural bond between sarcolemma and the deeper portion of the fiber (Figure $8 \mathrm{~A}$ and $\mathrm{B}$ ).

The number of $\mathrm{T}$-tubules varies among species and muscles. However, the distribution of tubules is similar within a given fiber. T-tubules consist of 2-4 free regions and junctional areas with $\mathrm{SR}$. These

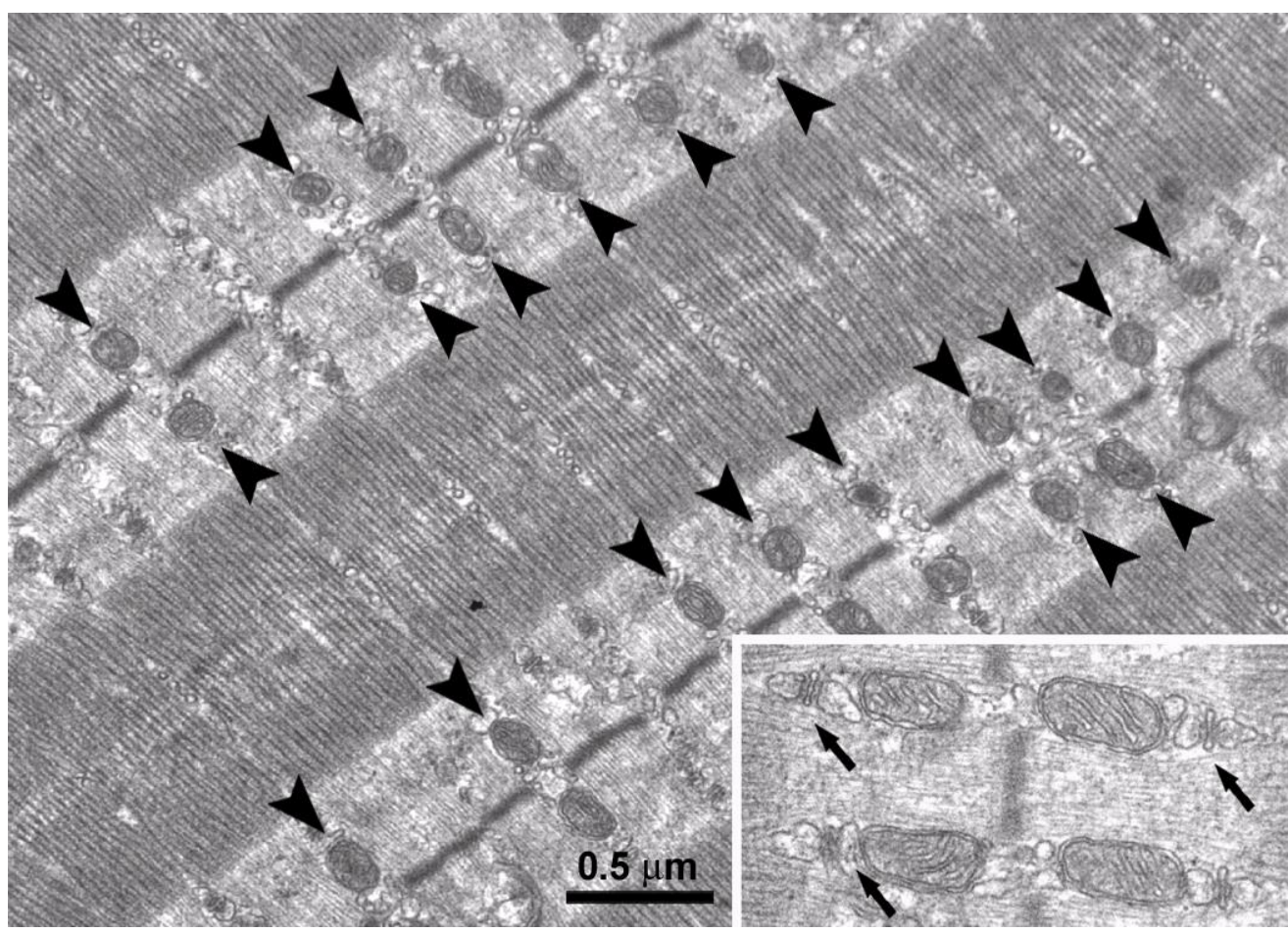

Fig 6 .Electron micrographs of FDB fibers obtained from 2 month old mice. Mitochondria are mostly found next to triads between the sarcomere A-I junction and the $Z$ line in the mature fibers (black arrowheads). Inset: under higher magnification, mitochondria are tethered to parajunctional regions of the SR (arrows). 


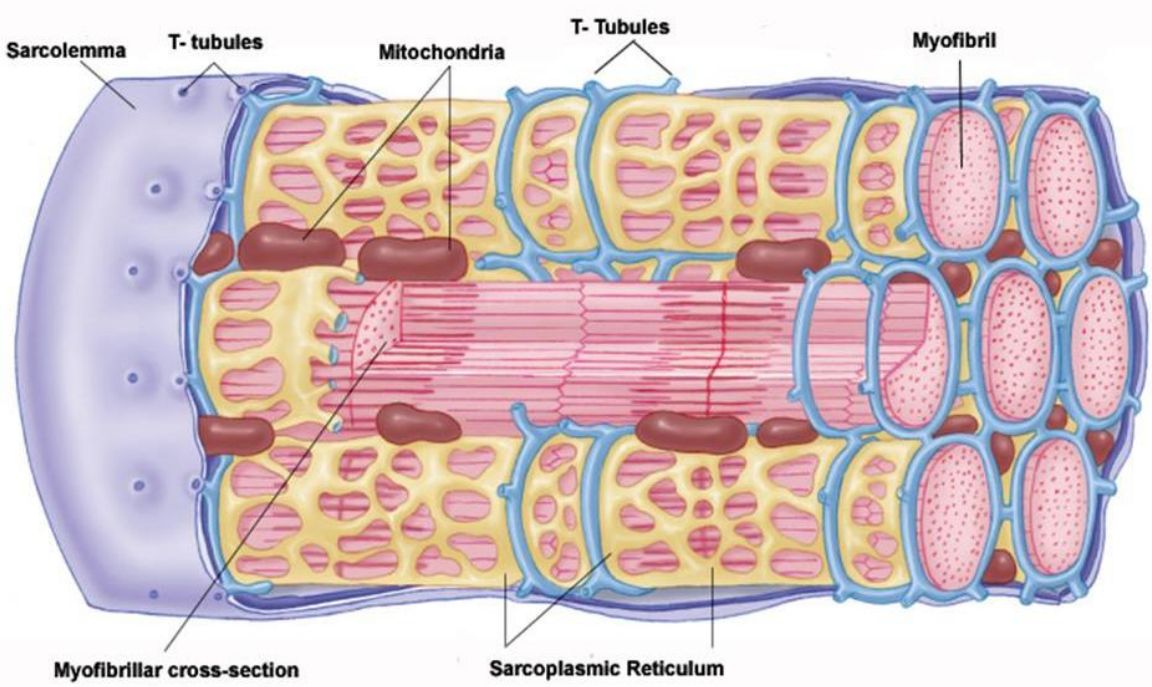

Fig 7 Schematic illustration of the membrane network of muscle fibers. The muscle fiber is enclosed by the sarcolemma. The membranous networks of the sarcoplasmic reticulum and transverse tubules are responsible for communicating the external stimulus provided by the motor neuron inward to the center of the fiber.

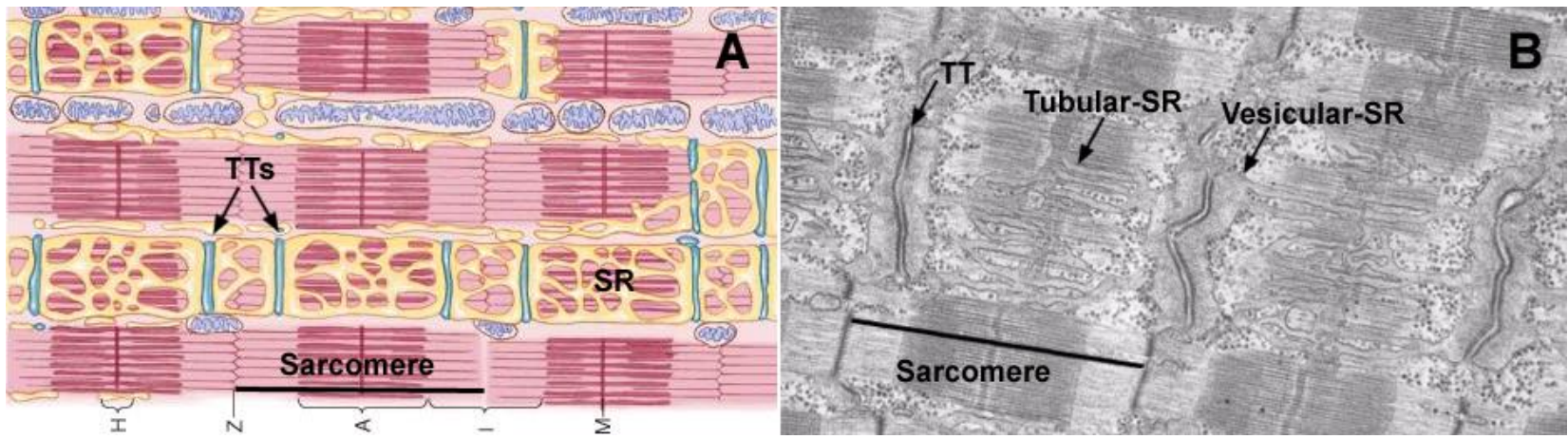

Fig 8. Organization of the Sarcotubular System. (A) Relationship between the transverse (T)-tubule and the sarcoplasmic reticulum (SR) with myofibrils. (B) An electron micrograph of skeletal muscle demonstrating the relationship between the precise register of fibrils and the SR/T-Tubule membranes. The prominent tubular component in the interfibrillar cytoplasm (sarcoplasm) is the SR. It is made up of interconnecting tubules and vesicles but in muscle it has no attached ribosomes. These reticular structures terminate in dilated sacs that are closely opposed to the interposed T-tubule system. This T-system is continuous with the sarcolemma (muscle plasma membrane). This enables myofibrils within the center of the fiber to contract simultaneously with those fibrils at the surface (Electron Micrograph, from Chapter 11 - Clara Franzini Armstrong - The membrane Systems of Muscle Cells. Myology 3ed).

specialized areas between the two membranes, also called couplings, contribute to the transmission of impulse from the depolarized T-tubules to the SR12.

1.1.3 Muscle Contraction and Excitation-Contraction Coupling (ECC).

Since the peripheral portion of the central nervous system (CNS) controls the skeletal muscles, thus, skeletal muscle contraction is generally initiated by the arrival an excitatory electrical signal at the neuromuscular junctions which is transduced into the physical interactions between the contractile proteins.
The mechano-chemical reaction between actin and myosin can only take place in the presence of myoplasmic free $\mathrm{Ca} 2+$ concentrations exceeding a threshold of 50-150 nM [13]. Muscle fibers are able to finely control cytoplasmic [Ca2+] thanks to the SR that sequesters and functions as a storage site for intracellular calcium [14]. The signal that activates muscle contraction is the sudden increase in intracellular $\left[\mathrm{Ca}^{2+}\right]$ that follows the depolarization of exterior membranes (sarcolemma/T tubules). This mechanism is named excitation-contraction coupling 


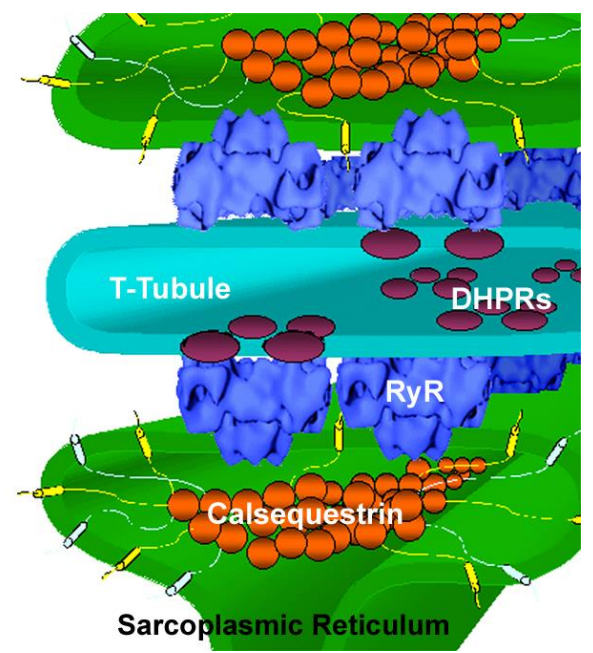

Fig 9 Schematic representation of the Calcium Realise Units in a skeletal muscle. A tridimensional reconstruction of a skeletal muscle triad showing the ultrastructural localization of RyRs, DHPRs, Calsequestrin, Triadin, Junctin (Graphic illustration, Courtesy of Feliciano Protasi).

(ECC) and takes place at calcium release units (CRUs) or junctions, those structures in which SR and Ttubules are closely associated with one another (Figure 9) $[15,16]$.

CRUs are found in both cardiac and skeletal cells and, while they are structurally quite similar in the two muscle types, they use two different mechanisms to accomplish the same goal: translating an electrical signal carried by the Tubules, into a $\mathrm{Ca} 2+$ release from the SR. In the heart, e-c coupling depends on the inward flux of $\mathrm{Ca} 2+$ through DHPRs, which triggers $\mathrm{Ca} 2+$ release from the SR stores [17].

\subsubsection{Ultrastructure of Calcium Realise Units}

Junctions between SR and sarcolemma/T-tubules are formed by specialized domains of the SR terminal cisternae (junctional SR) closely associated to junctional domains of exterior membranes. Junctions have different names (triads, dyads, and peripheral couplings) depending on the number of elements and the nature of membranes that constitutes them (Figure 10). Triads are formed by three elements, two SR terminal cisternae and one T-tubule, while dyads and peripheral coupling are formed by only two elements, one junctional SR and respectively a T-tubule or the surface membrane itself [15]. Whereas the different kinds of CRUs carry out the same function, it is possible to make a distinction on where and when they can be found. Triads are practically the only kind of junction present in adult skeletal muscle fibers, whereas dyads and peripheral coupling are the predominant type of CRUs in developing muscle and in the heart18. Striated muscles of invertebrates have all three types of junctions, but not necessarily in the

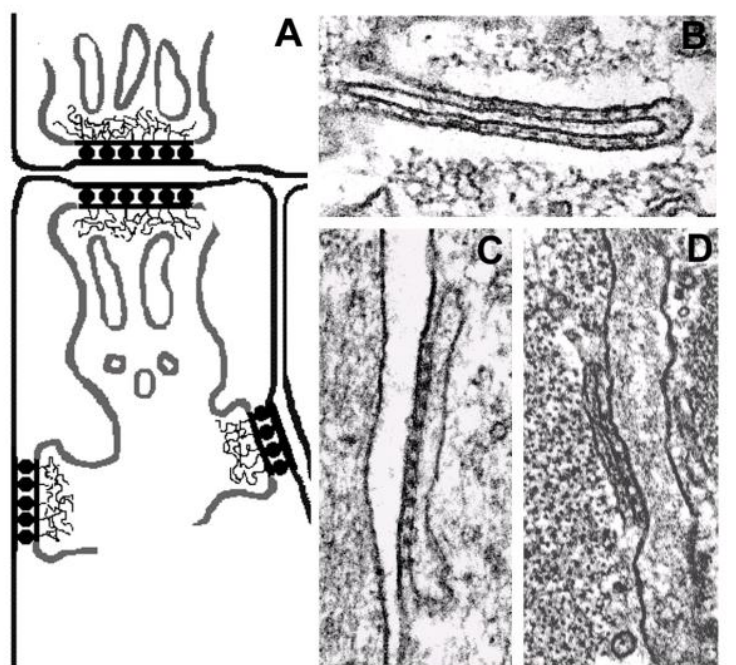

Fig 10 Different types of Calcium Release Units in muscle cells. CRUs, or junctions, are formed by the close apposition of SR terminal cisternae and exterior membranes. They are called triads, dyads, and peripheral couplings depending on the number and nature of the elements that constitutes them. A) Triads are formed by one T-tubule flanked by two SR cisternae (from adult toadfish swimbladder muscle). $B$ and $C$ ) Peripheral couplings and dyads are formed by only two elements: one SR vesicle and respectively the surface membrane or a $T$ tubule ( $B$, peripheral coupling in a $\mathrm{BC} 3 \mathrm{HI}$ cell; $C$, dyad in canine heart). The evenly spaced densities in the junctional gap between the two membranes have been identified with cytoplasmic domain of RyRs, the Ca2+ release channel of the SR. Bar, 0.1 um. (Picture, Courtesy of Feliciano Protasi)

same fibers. A different type of CRU has been also described in literature the extended junctional SR or corbular SR, found in interior of cardiac cells, and free of any association with external membranes [19].

In adult skeletal muscle triads are highly ordered and organized in the fiber and their position is strictly correlated to the striation of the myofibrils: either at the Z-line or at the A-I junction (Figure 11). This arrangement is achieved by gradual coordination of the CRUs and of the myofibrils during myogenesis $[15,18]$. This precise morphological relation between CRUs and myofibrils is essential for excitationcontraction coupling (ECC), the mechanism that allows transduction of the action potential into muscle contraction $[16,20]$.

1.1.5 RyRs and DHPRs: the two major players of ECC One of the first structures described as an integral components of triads are the feet (Figure $11 \mathrm{D})$, large electron-dense structures that bridge the narrow gap 

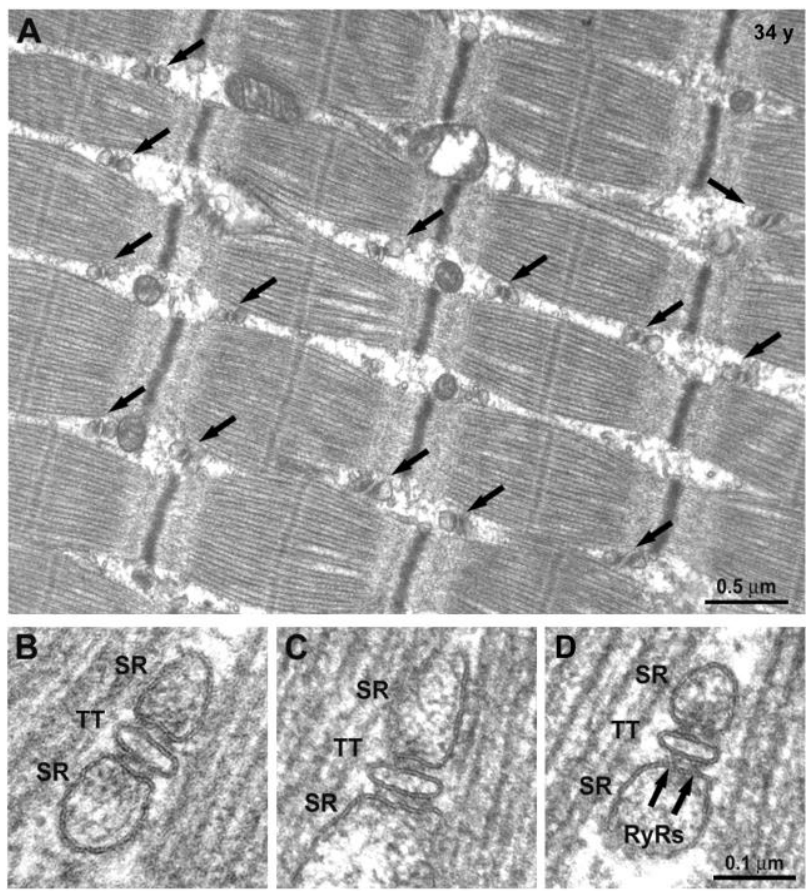

Fig 11 ECC apparatus in adult skeletal muscle: precise correlation between position of triads and striation of contractile apparatus. A) In mature skeletal muscle (vastus lateralis, 34 years of age, male), triads, indicated by the arrows, are usually located at sarcomere I-A junctions. The distribution of triads is fairly uniform within the fiber. $B$ and $C$ ) Higher magnification images provides a better view of the ultrastructure of skeletal triads. Two SR vesicles are apposed to a central $T$ tubule that usually have a flat profile. The junctional gap between the SR and $T$ tubule membrane is spanned by the cytoplasmic domain of RyRs (panel B), the feet 21 . In skeletal muscle, feet usually form two rows on each side of the T tubule.

(about 12nm) that separate the $\mathrm{SR}$ from the $\mathrm{T}$ tubule/sarcolemma [21].

Feet were later identified as the cytoplasmic domains of RyRs, and RyRs in turn were identified as the Ca2+ release channels of the SR [22]. The hydrophobic domain (the channel region) of RyRs is inserted in the SR membrane, leaving the large hydrophilic portion (foot region) in the cytoplasm. In electron micrographs, feet appear as evenly spaced densities in both skeletal and cardiac.

Another extremely important component of CRUs is the dihydropyridine receptors (DHPRs), an L-type $\mathrm{Ca} 2+$ channel that plays a central role in triggering SR $\mathrm{Ca} 2+$ release [23]. DHPRs are specifically localized in areas of exterior membranes that face junctional arrays feet in both skeletal and cardiac muscle fibers [24,25]. DHPRs are not as well visible as RyRs in thin sections, because they have smaller hydrophilic domains.
However DHPRs can be visualized in freeze fracture replicas, a technique that allows separation of the two membrane leaflets, exposing intra-membrane domains of proteins [26]. DHPRs appear as large particles clustered in correspondence of CRUs when visualized by freeze-fracture.

Despite the fact that many other proteins are involved structurally and functionally in ECC (i.e. calsequestrin, triadin, junctin, FKBP12, mitsugumin, junctophilin, etc.), RyRs and DHPRs are still recognized as the two key elements of the mechanism. In both cardiac and skeletal cells the key feature that allows the two proteins to interact with each other in a very efficient manner is their vicinity. DHPRs are always located in areas of exterior membranes that face RyR arrays of the SR, ideally placed to finely and promptly control the activation of $\mathrm{Ca} 2+$ release and start muscle contraction [20].

\subsubsection{Nerve and Neuromuscular Junctions}

Nerves, known as sensory neurons, come from every part of the body to carry impulses to the brain or spinal cord concerning the condition of every nook and cranny in the body, including the muscles. In the same way, motor neurons send impulses to the muscles, often through intermediate connections or "interneurons" in the spinal cord. These messages cross the gap at a junction between nerves and muscles, setting off a chain of events that ends in contraction. Within a second, millions of impulses reach the motor neurons. Some of the impulses are sent from various parts of the brain and spinal cord; some come from sense organs located in the joints, ligaments, and tendons; and some come from the muscles themselves. The seeds of movement are sown by the brain, in its primary cortex, an area of the brain's wrinkled surface which spans both cerebral hemispheres.

Another patch of cortex directly in front of the primary area also houses neurons which are involved in movement. This area is thought to be important to speech and delicately coordinated movements such as those performed by the hand. Electrical impulses from many regions of the brain feed into the motor areas. The brain must collect and analyze all the sensory messages it receives before it can direct a coordinated movement. This interplay is continuous and elaborate, sight, sound, smell, pressure and pain are all important, but are messages bringing information about the angles and position of joints, the length and tension of muscles, or even the speed of movements. At every point along the descent from brain to muscle, impulses can influence interneurons to vary the precision of muscular control. Control of muscle action is by the nervous system. Nerves are in touch with muscles by motor end plates which convey a stimulus to contract. Skeletal muscle fibers are supplied by motor nerves but usually only one axon (nerve cell) supplies one muscle fiber (this is not true for embryonic muscle where several axons might supply each muscle fiber but 

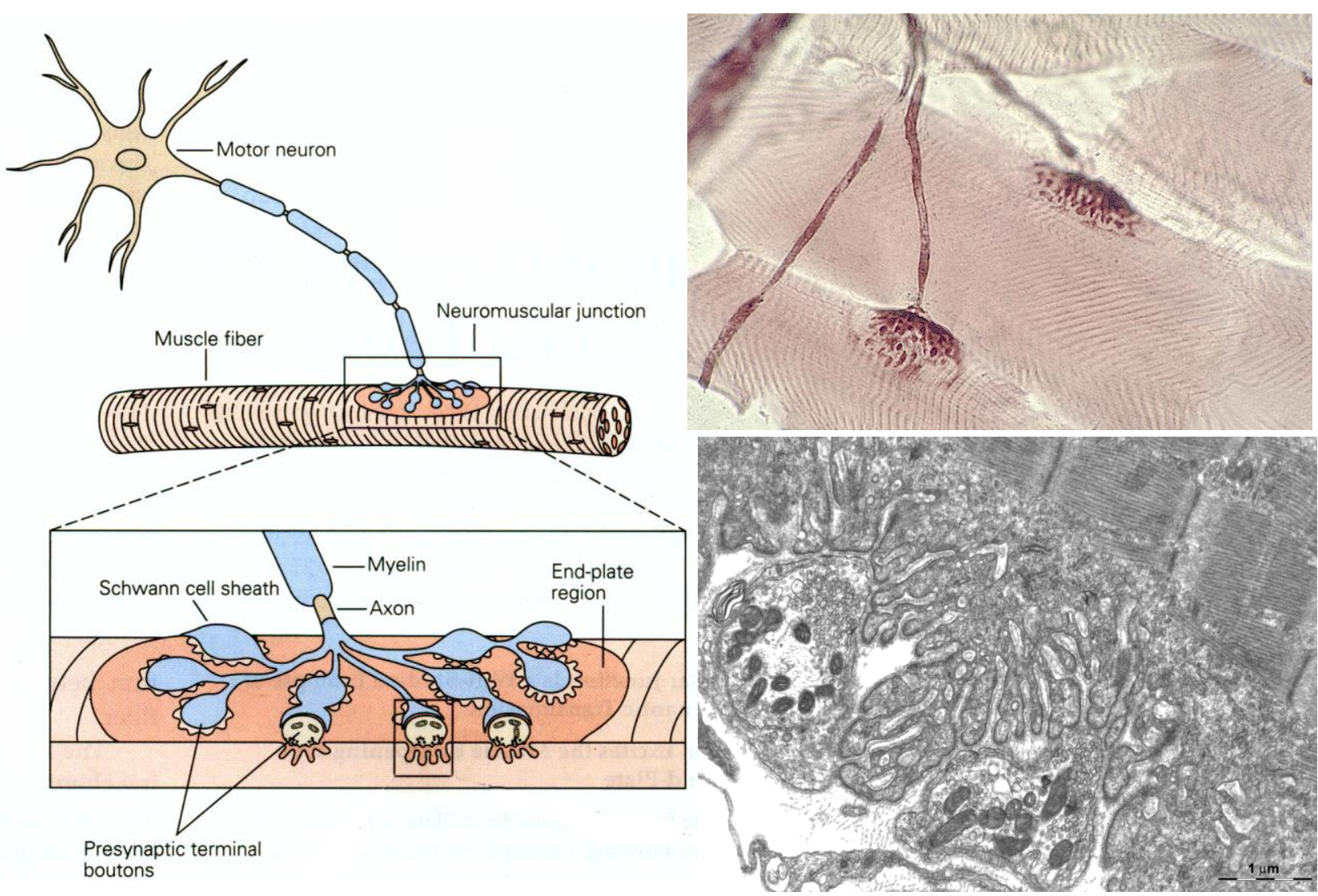

Fig 12 a) Graphic representation of neuromuscular junctions. b) Skeletal muscle and motoneurons in histological staining. c) Motor end plates as revealed by electron microscopy.

during the maturation of the muscle, only one axon remains to excite a single muscle fiber). Each axon usually divides into a number of terminal branches which lose their myelin sheaths and then each branch supplied a single muscle fiber.

The neuromuscolar junctions is a chemical synapse that is anatomically and functionally differentiated for the transmission of a signal from the motor nerve terminal to a circumscribed postsynaptic region on the muscle fiber [27]. The neuromuscular junction can be seen in the electron microscope where there is a small gap between the axon and the muscle membrane (Figure 12, C).

Nerves are also in touch with tendons via receptors which measure stretch and connective tissue by other receptors for pressure. These are referred to as efferent, if they are taking a message to a tissue and afferent if they are taking a message to the spinal cord and hence the brain. The brain and spinal cord is referred to as the Central Nervous System (CNS), and the connections as the peripheral nervous system .

\subsubsection{Trophic Interaction between Muscle and Nerve}

Neurons and skeletal muscle are both defined as excitable cells because of their capacity of carrying an electrical impulse, the action potential. Keeping into consideration this property, skeletal muscle may be considered a peripheral organ of the central nervous system from which it strictly depends for its structural and functional properties, during their normal development and maintenance [28-30].

Muscle fibers and the nervous system communicate in a bidirectional fashion, both during development and in adult life. This complex communication, which generally dependent on synaptic contact, based on electrical and chemical signals, has been proposed to be extremely important not only for the functionality of the system, but also for the maintenance of the entire neuromuscular apparatus [29,30]. Throughout life, motoneurons continue to exert important trophic influences on the muscles they innervate regulating many features of muscle fibers morphology and physiological properties, such as contraction kinetics and metabolic pathways $[31,32]$. Trophic regulation in the opposite direction is equally important, since also the motoneurons depend on the periphery for their survival and maintenance. The close interdependence between two systems becomes obvious in those cases in which their cross talk is interrupted. This may happen in a variety of neuropathological conditions, may be induced pharmacologically, or as a result of traumatic events such as spinal cord injuries (SCIs) or damage of peripheral nerves all causing the loss of 


\section{Muscle atrophy due to SCI can be reversed in complete absence of peripheral nerves}

European Journal Translational Myology - Basic Applied Myology 2012; 22 (4): 161-200

connection between muscle fibers and the axons that innervate them [33,34]. Although lack of motor control and gross muscle atrophy were the first recognized and most obvious alterations associated with muscle denervation [35-37], many other postsynaptic changes occur shortly after the injury. Denervation, or pharmacological interruption of normal synaptic activity, lead to rapid changes in many muscle parameters, with an overall tendency for muscle to revert to a state like that of embryonic fiber during which it regains the ability to accept innervation [38]. Denervation removes the supply of trophic substances from the nerve to muscle, stops muscle electrical and contractile activity, leads to a sharp drop in the rate of synthesis of muscle proteins and to an increase in the rate of degradation [39]. In addition, nerve damage also causes the degeneration of the distal stump of the nerve.

\subsection{SPINAL CORD INJURY}

\subsubsection{Spinal cord and the vertebra}

The brain and the spinal cord constitute the Central Nervous System. The spinal cord is about 18 inches long and extends from the base of the brain, down the middle of the back, to about the waist. The nerves that lie within the spinal cord are upper motor neurons and their function is to carry the messages back and forth

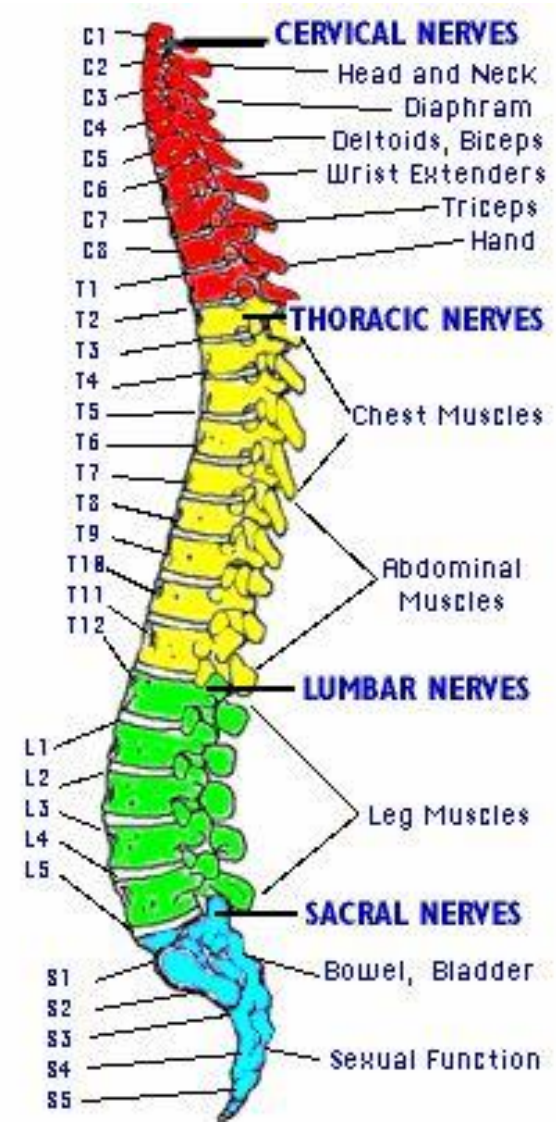

Fig 13 View of the spinal cord and the spinal nerves from the brain to the spinal nerves along the spinal tract. The spinal nerves that branch out from the spinal cord to the other parts of the body are called lower motor neurons. These spinal nerves exit and enter at each vertebral level and communicate with specific areas of the body (Figure 13). The sensory portions of the lower motor neuron carry messages about sensation from the skin and other body parts and organs to the brain. The motor portions of the lower motor neuron send messages from the brain to the various body parts to initiate actions such as muscle movement. The spinal cord is surrounded by rings of bone called vertebra. These bones constitute the spinal column (back bones).

\subsubsection{The Spinal Cord Injury}

Spinal Cord Injury (SCI) is a damage to the spinal cord that results in a loss of functions such as mobility and/sensitivity. Frequent causes of damage are trauma (car accident, gunshot, falls, etc.) or disease (polio, spina bifida, Friedreich's Ataxia, etc.). The spinal cord does not have to be severed in order for a loss of functioning to occur. In fact, in most people with SCI, the spinal cord is intact, but the damage to it results in loss of functioning.

The effects of SCI depend on the type of injury and the level of the injury. SCI can be divided into two types of injury: complete and incomplete. A complete injury means that there is no function below the level of the injury; no sensation and no voluntary movement. Both sides of the body are equally affected. An incomplete injury means that there is some functioning below the primary level of the injury. A person with an incomplete injury may be able to move one limb more than another, may be able to feel parts of the body that cannot be moved, or may have more functioning on one side of the body than the other. With the advances in acute treatment of SCI, incomplete injuries are becoming more common.

The level of injury is very helpful in predicting what parts of the body might be affected by paralysis and loss of function. Cervical (neck) injuries usually result in quadriplegia. Injuries above the $\mathrm{C}-4$ level may require a ventilator for the person to breathe. C-5 injuries often result in shoulder and biceps control, but no control at the wrist or hand. C-6 injuries generally yield wrist control, but no hand function. Individuals with $\mathrm{C}-7$ and $\mathrm{T}-1$ injuries can straighten their arms but still may have dexterity problems with the hand and fingers. Injuries at the thoracic level and below result in paraplegia, with the hands not affected. At T-1 to T8 there is most often control of the hands, but poor trunk control as the result of lack of abdominal muscle control. Lower T-injuries (T-9 to T-12) allow good truck control and good abdominal muscle control. Sitting balance is very good. Lumbar and Sacral injuries yield decreasing control of the hip flexors and legs. 


\section{Muscle atrophy due to SCI can be reversed in complete absence of peripheral nerves}

European Journal Translational Myology - Basic Applied Myology 2012; 22 (4): 161-200

Besides a loss of sensation or motor functioning, individuals with SCI also experience other changes such as dysfunction of the bowel and bladder. Sexual functioning is frequently with SCI may have their fertility affected, while women's fertility is generally not affected. Very high injuries $(\mathrm{C}-1, \mathrm{C}-2)$ can result in a loss of many involuntary functions including the ability to breathe, necessitating breathing aids such as mechanical ventilators or diaphragmatic pacemakers. Other effects of SCI may include low blood pressure, inability to regulate blood pressure effectively, reduced control of body temperature, inability to sweat below the level of injury, and chronic pain.

In general, the higher in the spinal column the injury occurs, the more dysfunction a person will experience. Cervical SCI's (in the eight vertebra of the neck) usually cause loss of function in the arms and legs, resulting in quadriplegia. Injuries in the thoracic region (in the twelve vertebra of the chest) usually affect the chest and the legs and result in paraplegia. This kind of paralysis is caused by a lesion in the region of the cauda equine, the lowest part of the spinal column. Specifically paraplegia was caused by traumatic fracture mostly of Th 11 and Th 12 (Th 5 -L1). In this case the lowest part of the spinal cord respectively the originating spinal roots are concerned and the resulting damage of the lower motor neuron leads to denervation of the more or less entire musculature of the lower body. Injuries to the five Lumbar vertebra (the vertebra in the lower back between the thoracic vertebra, where the ribs attach, and the pelvis; L-1 thru L-5) and similarly to the five Sacral Vertebra (from the Pelvis to the end of the spinal column S-1 thru S-5) generally result in some loss of functioning in the hips and legs. Approximately 450,000 people live with SCI in the US. There are about 10,000 new SCI's every year; the majority of them $(82 \%)$ involve males between the ages of 16-30. These injuries result from motor vehicle accidents $(36 \%)$, violence $(28.9 \%)$, or falls $(21.2 \%)$. Quadriplegia is slightly more common than paraplegia. Currently there is no cure for SCI. There are many researchers attacking this problem, and there have been many advances in the lab. Many of the most exciting advances have resulted in a decrease in damage at the time of the injury. Steroid drugs such as methylprednisolone reduce swelling, which is a common cause of secondary damage at the time of injury. The experimental drug Sygen ${ }^{\circledR}$ appears reduces loss of function, although the mechanism is not completely understood.

\subsection{EFFECTS OF DENERVATION ON SKELETAL MUSCLE}

The effect on muscle of a lack of innervation has been of primary interest in human pathology for many years. Since 1950s many scientist have been interested in the muscle alterations caused by diseases which lead to the destruction of motoneurons or to lesion of the peripheral nerves. Histological experiments have showed that following denervation, the most noticeable macroscopic change in a muscle is a rapid weight loss (atrophy) which is directly associated to the a significant decrease in the mean fiber diameter and an to an increase of fat and connective tissue [28,40]. Denervation-induced atrophy of muscle fibers can be looked upon as an adaptive mechanism for reducing the load in a functionless region or as a response of the muscle fibers to a poor local environment $[41,42]$.

Many of the studies that can be found in literature have been performed in animal models because of methodological limitations in the use of human material. In fact, in humans, a clear correlation between the alterations observed and the lack of innervation is physically not possible. The method of choice in most studies has been to cut the nerve supplying one or a group of muscles (mostly in rats) and to study the alterations occurring in the respective muscles at various time intervals after the operation. Franzini and Pellegrino (1963) [40], described by electron microscopy quite in detail the ultrastructural alterations of muscle fibers that follows denervation. Muscle atrophy is accompanied also by a significant disorganization of the internal ultrastructure of fibers, mostly evident in the loss of striation due to the disorganization of the contractile material. The appearance of the fibrils in longitudinal sections suggests that the process takes place by the detachment of filaments from the periphery of the fibrils and by their subsequent breakdown in the interfibrillary spaces. Recently, Takekura et al. (1999-2003) [43-45] described in detail the morphological changes that occur to the membrane systems involved with the excitation-contraction coupling (sarcoplasmic reticulum, transverse tubules and triads) in rat soleus and extensor digitorum longus muscle following a short term denervation [45]. The course of postdenervation events is more complex. In addition to muscle fiber atrophy, dramatic changes occur in the vascular bed, leading to a severe topographic dissociation of the muscle fibers from their capillary supply as well as sharply reduced capillary/muscle fiber ratios [46]. Significant myonuclear loss and some myofiber death occur as well [46,47]. A major accumulation of interstitial connective tissue following denervation has the potential of influencing the exchange of materials between the vascular bed and muscle fibres as well as the axonal growth during reinnervation.

While the effects of denervation on muscle are well recognized, what is not clear is which effects, and in which measure, are the result of the lack of communication between nerve and fiber or are instead the consequence of lack of activity. In fact, many procedures that reduce the activity level of motor nerves and muscles dramatically produce many of the effects of denervation [48]. In addition, it has been shown that direct chronic stimulation of denervated 


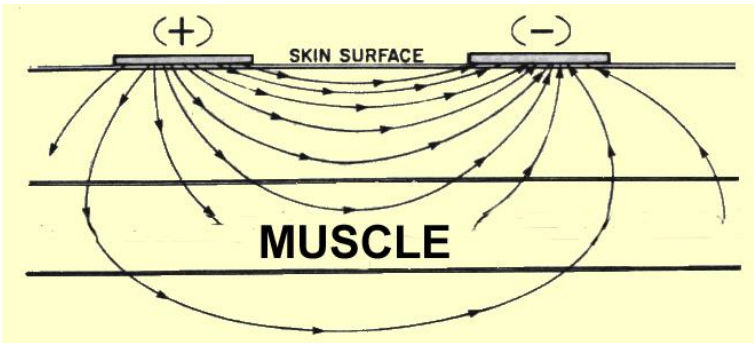

Fig 14 Illustration of a surface electrical stimulation: the current density diminishes with depth of muscle tissue.

muscle was remarkably effective in counteracting at least some effects of denervation $[39,49,50]$. On the other hand, there is compelling evidence that muscle membrane damage, nerve breakdown products, and block of axonal transport can lead to denervation-like changes in muscle that cannot be overcome by direct stimulation [51,52]. Many of these experimental manipulations are subject to ambiguities in interpretation and, while all those factors are likely influencing muscle properties, the relative importance of each in any given phenomenon is often difficult to establish.

\subsection{FUNCTIONAL ELECTRICAL STIMULATION \\ 1.4.1 Principles of FES Functional Electrical Stimulation}

Electrical stimulation is simply the application of electrical pulses to the body, be it for function or therapy. Functional Electrical Stimulation (FES) is a subset of electrical stimulation. The term FES is applied to systems which attempt to restore lost or impaired neuromuscular function, such as standing and walking in cases of paraplegia, by the application of electrical pulses to neural pathways, in this case FES is sometimes known as Functional Neuromuscular Stimulation or FNS, or, but less often, directly to muscles.

At the electrode-tissue interface a conversion occurs between the current of electrons passing through the wires and the current of ions moved within the tissue. Then through this externally applied current, the depolarization of nerve and muscle to threshold is produced by the transport of ions across the tissue membrane. The factors determining whether sufficient current flows to cause an action potential are:

- impedance of body tissues

- electrode size and position

- stimulation parameters

The conductivity of body tissues is related to their water and ion content. Muscle is a good conductor, but conducts much better in the longitudinal direction of its fibers than in the transverse one, while adipose tissue is a good insulator. Thus electrode position can affect the current required for stimulation. With surface electrical stimulation the effect of the electric current (the current
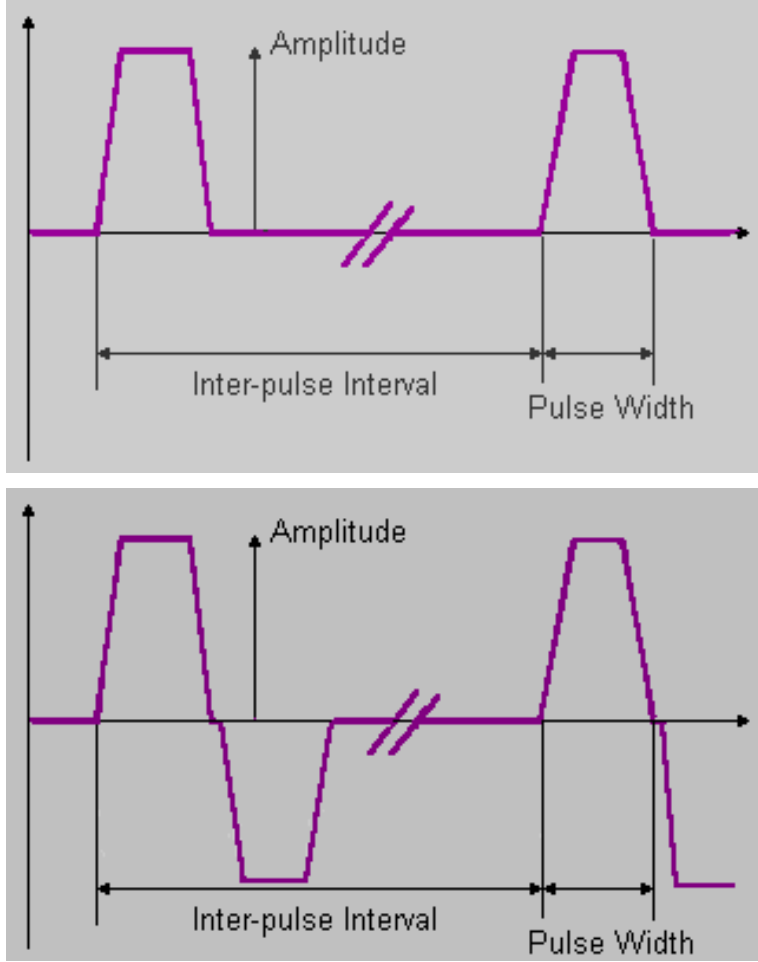

Fig 15. Graphic representation of monophasic (upper panel) and of biphasic pulses (lower panel), respectively

density) diminishes with depth of tissue, as illustrated in Figure 14. FES involves depolarizing nerve/muscle fibers via externally applied electric current. Once depolarized these fibers conduct action potentials as occurs in healthy tissue. The electrical pulses applied may be: monophasic or biphasic (Figure 15). In the second case, when charge balanced pulse types are used, no net charge is introduced to the body. This is particularly important for some types of denervated muscle stimulation and for implanted electrodes, where electrolysis at the electrode tissue interface could take place if there was a non-zero net charge.

Surface electrical stimulation typically consists of a train of regular monophasic or biphasic pulses which may be described by the following parameters: frequency; amplitude; duration of individual pulses; duration of the pulse train; and rise time for the individual pulses

\subsubsection{FES on patients with upper motoneurons lesion (spastic patients).}

Patients that have lesion of the upper motoneruon, stillThe amplitude and pulse width of the stimulation must be sufficient to meet the threshold of excitability of the stimulated tissue, changes under this level will have no effect. As the amplitude or pulse width rise the nerve fibers nearest the electrodes of directly and largest in diameter are triggered to threshold and fire. This continues until all fibers are firing at which point no more increase in force can be obtained, the muscle 


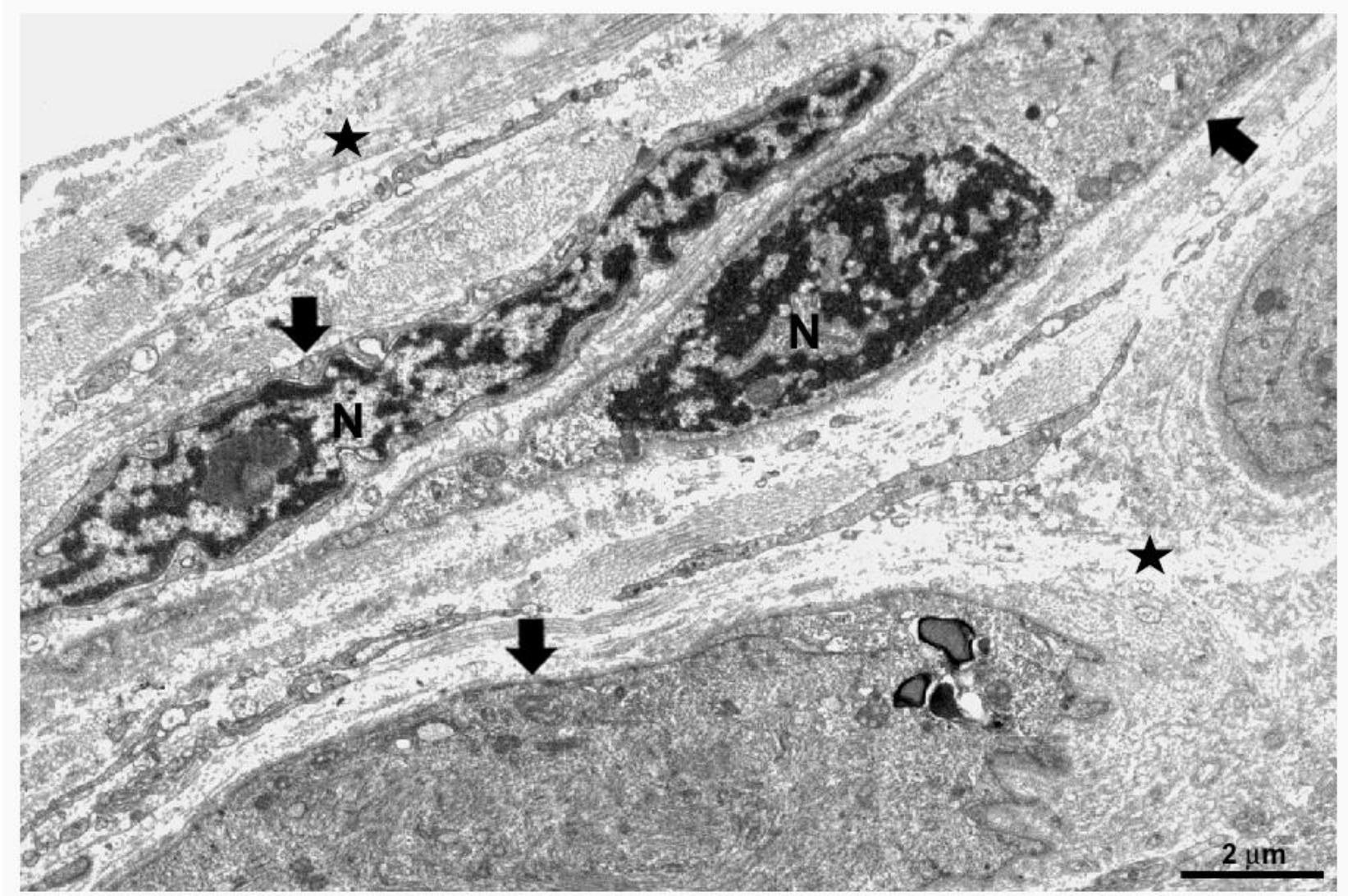

Fig 16 Low magnification image of a 4 year denervated muscle (vastus lateralis). Fibers that have been denervated for very long periods of time are severely atrophic. Their diameter is extremely small and the striated appearance, characteristic of skeletal muscle tissue, is disappeared. Black arrows points at the surface membrane. The extra cellular space (asterisks) is usually enlarged and filled with collagen.

is said to be saturated. The rate of rise of the pulse can also be important. Too slow a rise time results in changes in the tissue membrane known as accommodation, which gradually elevates the threshold required for the nerve to fire. The pulse used in electrical stimulation do not, in general, allow this effect to occur. The rate at which the nerve fibres fire is dependent on the frequency of pulse repetition. A single pulse produces a short lived muscle twitch of not more than $250 \mathrm{~ms}$. If pulses are repeated more frequently than this the muscle does not have time to relax in-between stimuli and eventually tetanic (continuous) contraction occurs. Although these look similar to contractions evoked by voluntary stimuli, as voluntary motoneurons are innervated asyncronously, tetanus is achieved at much lower rates $5-25 \mathrm{~Hz}$.

1.4.3 FES on patients with lower motoneuron lesion (completely denervated patients).

Practically all established clinical FES applications are based on direct excitation of neural structures and in case of muscle functions indirect activation of the muscle. For the functional activation of denervated and degenerated muscle (DDM) the technical requirements differ substantially from those for nerve stimulation.
Due to the absence of neuromuscular junction and decomposition of motor units muscular contractions can only be elicited by depolarizing the cellular membrane of each single muscle. The electrical membrane excitability strongly depends on the state of degeneration or restoration of the muscle cell but in any case it is much lower than the excitability of a nerve cell. At first, in order to achieve contractions, in DDM biphasic rectangular impulses with duration between 30 and $300 \mathrm{~ms}$ have to be applied. Consequently, also the required amplitude values are significantly higher than for comparable nerve stimulation. The recruitment of a sufficient fiber population is depending on a homogenously distributed electrical field more or less concentrated on the target muscle. Biphasic rectangular impulses are the most efficient impulse shape for FES in DDM

\subsection{SCIENTIFIC MEANING OF THE PRESENT STUDY}

While the effects of denervation on muscle have been widely studied in animal models, not much as been done in humans $[53,54]$ especially at longer times from injury. In the first part of the present study we have analyzed seven human muscle biopsies from patients 


\section{Muscle atrophy due to SCI can be reversed in complete absence of peripheral nerves}

European Journal Translational Myology - Basic Applied Myology 2012; 22 (4): 161-200

affected by complete conus cauda lesion, i.e. patients that have no peripheral nerves. Our structural studies illustrates: a) the different aspect of denervation (Figure 16) and the time course of degeneration of muscle fibers and parallel were made with animal models previously studied.

In the second part of our study, on the other hand, we have investigated the capability of long-term denervated muscle to recover under the influence of FES delivered with specially designed apparatuses and protocols.

The most important question that has driven research in the last years is whether or not an injured spinal cord can be repaired. In the last years significant breakthroughs in a number of SCI fields have led to the recognition that SCI patients do have reasons to hope [55-57]. However, what seems to be still a quite difficult task is to reconnect axons to peripheral targets. In fact, it has been very well shown that axons will reinnervate peripheral organs if the distances and time lapsed after injury are short, e.g., when short segments of the motor axon have been damaged, but do not reconnect efficiently over longer distances and when longer periods have elapsed for the injury event [58-61].

In the treatment of long-term SCI patients there are some very relevant questions that would be important to answer to improve quality and possibly increase chances of a functional recovery: is it possible to reverse muscle wasting and recover muscle tissue in long-term denervated patients? Should strong measures be taken early in the injury treatment, in order to reduce atrophy, with the hope of facilitating possible re-innervation events?

In literature there are many papers clearly indicating that directly evoked muscle activity could prevent or reverse many of the effects of denervation [39,49,6265]. However, the majority of stimulation studies were performed in animal models and after a relatively short time of denervation. For humans, in fact, the common belief in the clinical communities is that no effective treatment is available to rescue human muscles that have undergone severe atrophy as a result of a longstanding denervation injury $[66,67]$. However, contrary to this common belief, an innovative rehabilitation procedure based on functional electrical stimulation (FES), has recently proved to effectively reverse longlasting muscle atrophy in the muscles of the lower extremities of SCI patients affected by complete lesion of the conus cauda, i.e. that have no peripheral nerve endings [68-70]. Experimental and clinical results have shown that this FES training based on specifically designed stimulation devices and protocols can efficiently restore muscle mass, force production and movements even after long lasting complete denervation (up to 2 years). Measurements by CTscans revealed a substantial increase of tight muscle cross sectional area during the first years of FES, and muscle function of the lower extremities was restored in some patients sufficiently to allow for supported standing, and even for a few steps to be taken under indirect electrical stimulation [68,71]. A detailed analysis of the histological aspect of muscle recovery was reported in Kern et al. 2004 [72], showing a great increase in average diameter of muscle fibers following FES treatment, accompanied by a great reduction of collagen and adipocyte accumulation between fibers. A brief preliminary description of the ultrastructural recovery of muscle fibers was also presented (Kern et al., 2004) [72], but only based on two patients and few fibers analyzed. In the present work we have analyzed using transmission electron microscopy (TEM), 10 human muscle biopsies from 10 different patients, five of which treated with FES for prolonged periods of time (2.4 to 9.3 years), that all suffered complete lesion of the spinal cord. The capability of severely atrophic muscle fibers to recover and the mechanisms that allows such recovery were studied in each of the biopsies analyzing structural parameters such as the level of organization of the contractile apparatus, the ECC apparatus, mitochondria, and their reciprocal positioning in correspondence of the sarcomeres striation. Parallels were made between this structural recovery occurring under the influence of FES-induced muscle activity and in complete absence of nerve endings, with those that take place under normal muscle differentiation as well as recovery after short-term disuse

\section{MATERIALS AND METHODS \\ 2.1 PATIENTS' CHARACTERISTICS}

All subjects studied in the present work (all males, ages ranging from 27 to 58) had experienced complete traumatic conus cauda lesion. None of the subjects presented any other neurological disorders or muscle diseases besides the SCI. The patients were carefully tested to assess the complete lack of innervation of the quadricept muscles since they had a conus cauda lesion femoris for at least 6-12 months. These patients, who met the inclusion criteria were invited to Vienna. They were informd about the project and had to give their informed consent. Before to starting the electrical stimulation program the patients had to undergo a series of examinations to prove that they met the inclusion criteria (complete denervations of $\mathrm{m}$. quadriceps femoris with absent voluntary movement, sensation and reflexes) and to describe the status of the denervated muscle at the onset. Test stimulation, clinical and neurological examinations, neurophysiological assessment, biopsies of skin and quadriceps muscles, computerized tomography scans (CT scans) of the thighs, bone density measurements, skin examinations and mechanical evaluations were carried out. For the test stimulations the patients was sitting with extended lower limbs, two pairs of large electrodes $(200 \mathrm{~cm} 2)$, each inside a wet sponge bag, 


\section{Muscle atrophy due to SCI can be reversed in complete absence of peripheral nerves}

European Journal Translational Myology - Basic Applied Myology 2012; 22 (4): 161-200

positioned above his thighs. The quadriceps muscle was stimulated with biphasic rectangular impulses of defined durations $(145,42,5,2.6$ and $1.3 \mathrm{~ms})$ and a maximum intensity of $160 \mathrm{~V}$ peak to peak (Vpp). By palpating the muscle belly and the patella it was decided if a contraction of the stimulated muscle could be elicited. Only patients whose quadriceps muscle contracted by applying $5 \mathrm{~ms}$ or longer lasting impulses were included. A detailed description of the functional testing performed (Chronaxie measurements, Needle EMG, Brain Motor Control Assessment, Transcranial and Lumbosacral Magnetic Stimulation) can be found in Modlin et al., 200571.

\subsection{CLINICAL TESTING OF PATIENTS}

2.2.1 Determination of muscle cross-sectional area of thigh muscles by CT scan

Since thigh muscles are more or less spindle-shaped, the CT-cut plan ought to be well defined, so that results could be compared. We use as reference points the tops of both trochanters, which are determined by CT scan. Preventing torsion of body axis, the reference line is established by linking the two trochanter tops. Results are reproducible within a single thigh, as well as in right vs. left leg comparison in each patient. All patients are lined supine on the table ("Feet-first position") parallel to the table axis. The first body section is established at the tops of the trochanteres maiori. Three additional thigh sections are performed distally every $100 \mathrm{~mm}$. To clearly distinguish fat from skeletal muscle tissue a soft window frame (window 350 , center 50) is used. Beside complete cross section area of the upper thigh the cross sectional areas of $\mathbf{M}$. gluteus, M. quadriceps and hamstrings, as well as their density, are determined. The cross- sectional areas of muscle quadriceps femoris and the hamstrings were measured in $\mathrm{cm}^{2}$ and/or as percent of base line value, the density of these areas was measured in Houndsfield units (HU).

\subsubsection{Force Measurements}

Force of the quadriceps muscle is measured during electrical stimulation as torque of extension movement of the knee. The measure is performed in sitting position using a purpose-designed chair where subjects sit with the legs in $90^{\circ}$ knee flexion position. A dynamometer fixed between the chair and the leg measures force of the quadriceps muscle during electrical stimulation. As an index of muscle trophism and of the efficacy of the training program, force of the thigh muscles is measured as extension torque and expressed in $\mathrm{Nm}$, using increasing stimulation amplitudes from 0 to $160 \mathrm{Vpp}$ in $10 \mathrm{~V}$ steps. The optimal stimulation parameters are determined in each patient by varying the impulse widths $(\mathrm{msec})$ at after complete conus cauda lesion.

\subsection{MUSCLE BIOPSY}

\subsubsection{Needle muscle biopsy}

Used since 1868 (Duchenne) and re-popularised by Bergstrom (1962) and Edwards (1971). Involves the insertion of a hollow bored needle under local anaesthetic and sterile conditions to obtain specimens around $20-40 \mathrm{mg}$ containing approximately 100-700 muscle fibres. The needle muscle biopsy represent the preferred method nowadays.

Procedure:

- Anaesthetized skin and subcutaneous tissue with $1 \%$ lignocaine (avoiding contact with muscle)

- Incise skin and deep fascia with scalpel blade

- Insert needle minus central rod

- Press muscle bulk into needle side-window

- Cut off sample by ramming inner (sharpened) cylinder along needle

- Remove needle and use central rod to evacuate specimen

- Close wound and seal skin with elastoplasts

Advantages:

- Useful for patients with respiratory problems

- Useful for children

- Repeat biopsies convenient

- Little scarring

- Cost-effective

Disadvantages:

- Small specimens

- Blind procedure

2.3.2 Biopsy Specimens

After a small skin biopsy was taken ( $6 \mathrm{~mm}$ diameter), needle muscle biopsies were harvested from both the right and left vastus lateralis muscle of the muscle quadriceps femoris. The resulting specimens were then prepared for light and electron microscopical analysis.

\subsection{ELECTRICAL STIMULATION TRAINING}

After passing the initial examinations, some patients started their electrical stimulation training. The training was carried out at home after appropriated instruction in stimulating not only the quadriceps muscle but also the gluteus muscle and calf bilaterally.

Electrical stimulation was applied by a specially developed stimulation device with large electrodes ( $200 \mathrm{~cm} 2)$ in sponge bags which were placed over the muscles. After 4-6 months, when the skin had adapted the electrical stimulation, the electrodes on the thighs were applied to the skin directly with gel. Every four to eight weeks accurate checks ups and appropriate adaptations of the stimulation protocol were made. Depending on the results of the test stimulations (impulse duration necessary to elicit a muscle contraction) the electrical stimulation program was set up. Sometime to elicit a muscle twitch in denervateddegenerated muscle by surface electrodes, it is necessary to apply an extremely long biphasic rectangular stimulus of 150-200 ms duration and of up to $200 \mathrm{~mA}$ amplitude. No commercially available stimulators can deliver such a high current intensity, and therefore a generator of long and high-strength stimuli was designed and developed [70]. The output stage provides four different charge-balanced impulse forms (1 biphasic rectangular or 3 biphasic triangular). 


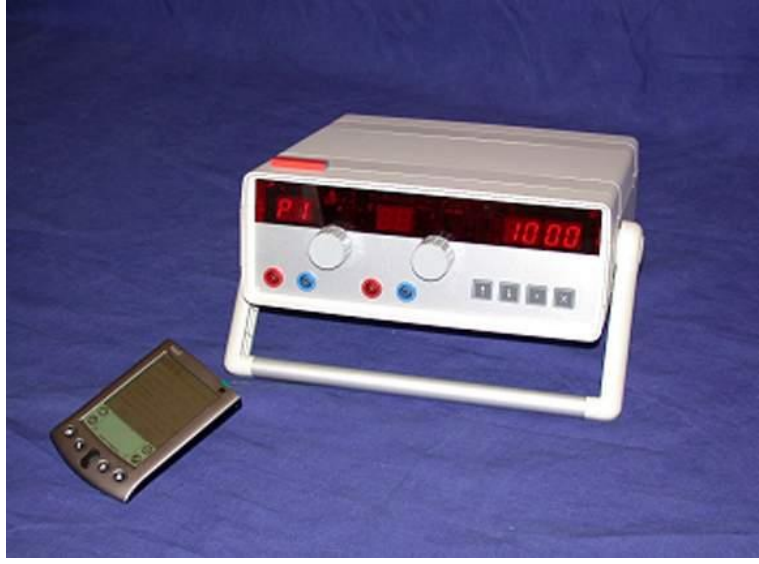

Fig 17 FES Stimulation Device

In addition, to prevent occurrence of direct current due to inaccurate charge compensation the stimulation pulses are capacitively coupled (Figure 17). The microprocessor-based design provides flexibility regarding the generation of the required stimulation parameters. This allows additional features, i.e., documentation of the stimulation program the patient performs (date, time, duration and stimulation parameters of each training session).

There are two reasons to stimulate denervated degenerated muscles with anatomically shaped, large size electrodes. First, the lack of excitable motoneurons imposes direct activation of the myofibers, which only depolarize if the difference in electrical potential is sufficiently high. Thus, the electrical field ought to be homogeneously distributed all over the whole muscle. Second, to safely apply very high stimulation currents for activating the denervated muscle fibers, the electrodes ought to be large enough to keep the current density at a low range to prevent skin damage. For surface stimulation we recommend electrodes made of silicone-graphite, which are applied directly to the skin using a wet sponge cloth (at the beginning of training) or gel (later on, when skin trophism has improved). It is important to use flexible electrodes that accommodate to the uneven skin surface to provide homogenous contact and thus homogenous distribution of the electrical field in the stimulated thigh (Figure 18).

The implantable device consists of a battery-powered programmable stimulator connected via leads to a pair of epimysial electrodes. The external components are a notebook computer and a transmitter/receiver unit. The stimulator generates biphasic, constant-current pulses. All pulse and burst parameters can be specified on a graphical user interface on the notebook computer; the program is then transferred to the implanted device via a bi-directional radio-frequency link. The device is suitable for intra-peritoneal implantation in experimental animals and it had been proved reliable

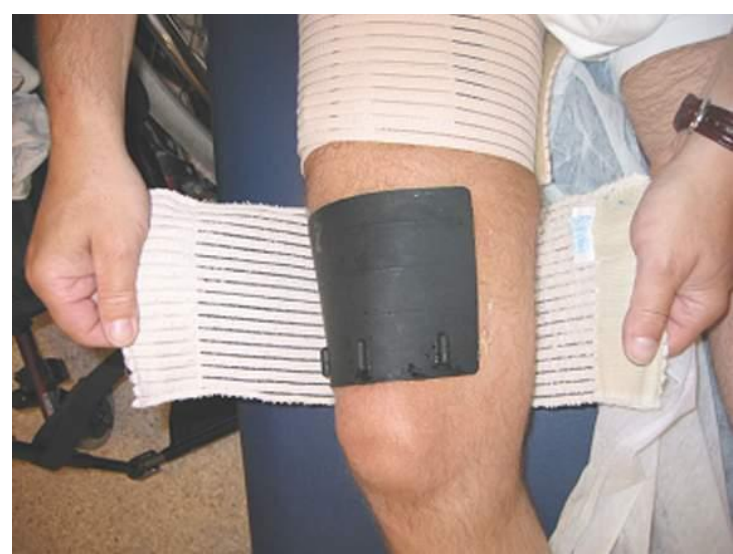

Fig 18 Surface Electrode

and stable in preliminary experiments in rabbits. Pulse generator volume and electrodes shape will be adjusted in size depending on the animal model (rats or rabbits). The devices will be produced by the research group FES Implants that is a facility of the Centre of Biomedical Engineering and Physics of the Medical University of Vienna, specialized in the development of implantable devices.

\subsubsection{Rehabilitation Training: stimulation parameters and protocols}

Functional response of DDM to electrical stimulation depends on the stage of post-denervation muscle atrophy/degeneration, which in turn depends on the time period between denervation event and stimulation onset. The minimal effective stimulation current depends on extent of degeneration of the stimulated muscle.

\section{Phase I: Muscle Contraction: Early Twitch Stimulation; Months of Training: 1-4}

In these patients, from 1 year and up to 3 years after injury, at the beginning of the treatment we applied biphasic rectangular stimulation impulses with very long duration and high intensity to attain single twitch contractions of thigh muscles. First, we assessed the severely reduced excitability of long-term denervated myofibers (long-term complete spinal-motoneuron denervation) by delivering very long biphasic rectangular impulses, which, however, yielded only twitch contractions of the thigh muscles. Twitch contractions were elicited by biphasic rectangular current pulses lasting 150 to $200 \mathrm{~ms}$ (this is about 1500 times longer than in spastic paralysis patients, in which the motor neurones are preserved) and up to $\pm 200 \mathrm{~mA}$ amplitude, representing an impulse energy up to 3.2 Jouls, to recruit fibers throughout the quadriceps femoris muscles. This strong stimulus is able to elicit single twitches of the degenerated-denervated muscle (DDM). With an interpulse interval of about $400 \mathrm{~ms}$ the resulting stimulation frequency is slightly less than $2 \mathrm{~Hz}$ ("single twitches" elicited every half second). 

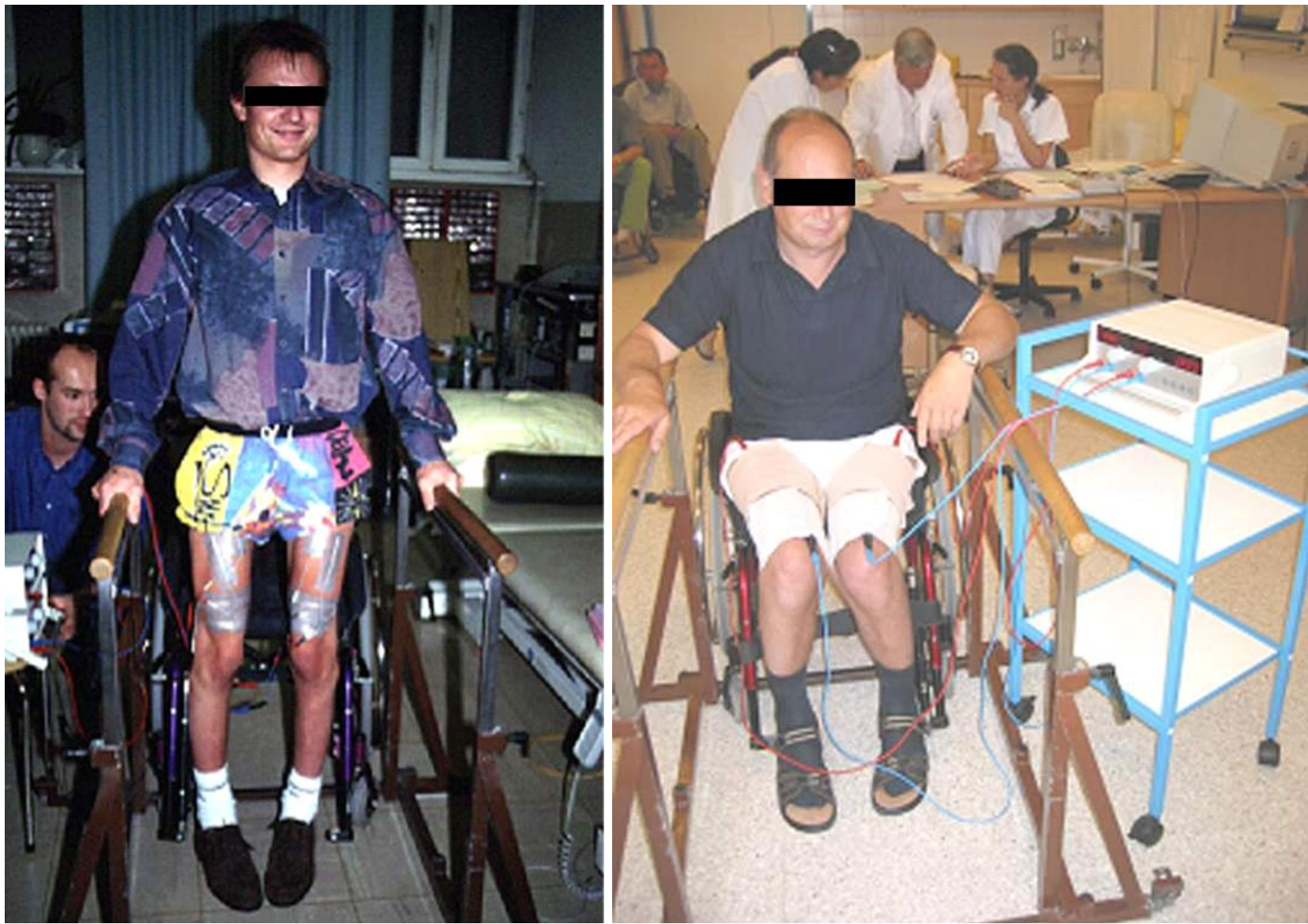

Fig 19 Stand up and sit down exercises in parallel bars.

This Training initiated at $2 \mathrm{~Hz}$, is delivered for 15 $\mathrm{min} /$ day (series of $4 \mathrm{~s}$ "on", $2 \mathrm{~s}$ "off"), 5 days/week. During the next few months, the progressively increasing muscle excitability permitted an increase of the twitch stimulation to series of $5 \mathrm{~s}$ "on," $1 \mathrm{~s}$ "off," 3 to $5 \mathrm{~min}$ of stimulation with 1 to $2 \mathrm{~min}$ of rest. Since no stimulator on the market could deliver such a high current intensity, has been developed a generator of long, high-strength stimuli [70,73].

Phase II: Muscle Contraction: single twitch and first tetanic contractions; Months of Training: 2-6

One effect of the electrical stimulation program is the increased excitability of the muscle fibers. Therefore during the successive 3 months of training, the pulse duration and the inter-pulse interval could be accordingly shortened to 80 to $100 \mathrm{~ms}$, and to increase the number of stimuli per second delivered to the patient muscles.

Phase III: Muscle Contraction: tetanic contractions and knee extension; Months of Training: 4-12

After Once strength of muscle contraction increases in consequence of significant structural and metabolic improvements of muscle tissue, the stimulation impulses are shortened to $50-35 \mathrm{~ms}$ (off-time $10 \mathrm{~ms}$ ) to raise impulse frequency to $16-25 \mathrm{~Hz}$.
Phase IV: Muscle Contraction: tetanic contractions, knee extension with increasing ankle weight; Months of Training: 6-12.

Phase V: Muscle Contraction: force endurance training. Months of Training: 8-12

Between the 8th and 12th month of FES training of denervated muscles, force-training sessions were introduced by tetanic contractions with 70 to 80 percent of maximum load, 8 to 12 repetitions, 4 to 6 sets, with 2 min of rest, twice a week. At first, the leg contracted to full knee extension without any ankle weight, and later, with an ankle weight of up to $5 \mathrm{~kg}$, in $0.5 \mathrm{~kg}$ steps. With this progressive FES training, the mass and force of thigh muscles increased to values that allowed electrical-stimulation-supported standing up and standing exercise (See Phase VI and VII).

Phase VI: Muscle Contraction: virtualization, standing up. Months of Training: 12-18.

Phase VII: Muscle Contraction: "stepping like" exercises. Months of Training: 12-24.

\subsubsection{Daily Therapy and Training Time}

The daily therapy program requires individual stimulation of m. gluteus, m. quadriceps, hamstrings and $\mathrm{m}$. triceps surae for 15-20 minutes each muscle (in 3-5 series of 3-4 minutes) once or twice a day. Patients 


\section{Muscle atrophy due to SCI can be reversed in complete absence of peripheral nerves}

European Journal Translational Myology - Basic Applied Myology 2012; 22 (4): 161-200

use a stimulation device with two independent channels to simultaneously stimulate left and right side muscles. The FES training takes approximately two hours a day (including time for donning and doffing the electrodes). At the beginning, training is carried out in sitting position with extended legs (with or without foam roll) and then with $90^{\circ}$ knee flexion. The stimulation protocols with both single twitches and early tetanic contractions are done without added load. Later on, while knee extension torque continues to increase, ankle weights are used to increase training intensity. When the developed force in the leg muscles is sufficient to stabilize the knee joint in standing position, that is, the knee extension torque is higher than $20 \mathrm{Nm}$, the so-called functional training can start. The patient performs standing up exercises and simulates gait by alternately switching on and off muscle stimulation for the left and right leg in the upright position in parallel bars (Figure 19). Usual outcome of our electrical stimulation program is the ability to extend the knee joint in sitting position after 4-6 months, and the ability to stabilize the knee joint in standing position after 12 months. Then standing up exercises became possible, including initiation of gait simulation by manual control of the stimulation program. Beside by clinical observations, effects of the stimulation program are measured by muscle cross sectional area with CT scans and by knee extension torque.

\subsection{LIGHT MICROSCOPY}

Serial sections are cryosectioned from samples obtained by needle biopsies frozen in isopentane cooled with liquid nitrogen. For histology or immunohistochemistry, sections of $10 \mu \mathrm{m}$ thickness are collected on polylysinated glass slides. For molecular analyses, a precise number (usually two) of sections of $20 \mu \mathrm{m}$ thickness are transferred to eppendorf test tubes. The slides and test tubes are stored at $-80^{\circ} \mathrm{C}$ until use. Three $10 \mu \mathrm{m}$ thick sections are collected on glass slides and stained using conventional techniques with Hematoxilin and eosin (H\&E). Total area of the slide and percent areas covered by myofibers, interstitial and fat tissues are determined in H\&E-stained sections as described below (morphometric analyses). Fiber counts to determine fiber type distribution are based on myofibers identifiable in H\&E-stained sections, in which the smallest myofibers are hardly recognized. In long-term denervated muscle total myofiber counts and their fiber size distribution could be determined using semi-thin sections (see below).

\subsubsection{Hystology}

a. Hematoxilin-eosin, Oil red $\mathrm{O}$ and Mallory trichromic stains. Cryosections (10 $\mu \mathrm{m}$ thick) of frozen biopsies were stained with hematoxilin-eosin (H-E), oil red $\mathrm{O}$ stain for lipid displaying and the trichrome stain (Mallory) for demonstration of distribution of collagen, using conventional techniques. b. Immunohistochemistry. Cryo-sections were labeled with anti-MHC-emb antibody (from Novocastra, NCLMHCd diluted 1:20) for 1 hour at room temperature. The slides were then washed twice with TBS (5 min each) and incubated with FITC-conjugated anti-mouse Ig (from Sigma, F-2266 diluted 1:200) for $1 \mathrm{~h}$ at room temperature. This was followed by a second 5 minute washing of the slides with TBS and nuclei counterstaining by Hoechst 33258. In the negative controls, the primary antibody was omitted.

\subsection{ELECTRON MICROSCOPY}

Needle muscle biopsies were harvested from both right and left vastus lateralis muscles at a single time point for each patient. Time elapsed from injury to the biopsy procedure are reported for each patient the various Tables. Samples were fixed in $2.5 \%$ glutaraldehyde in a $0.2 \mathrm{M}$ sodium cacodylate buffer, $\mathrm{pH} 7.2$ for $2 \mathrm{~h}$. The samples were kept in fixative solution for no longer than 3 days before the embedding procedure. Small pieces of tissues (untreated patients) or small muscle bundles (FES treated patients) were post-fixed in for $1 \mathrm{~h}$ in $1 \%$ osmium tetroxide in the same buffer, dehydrated in a graded series of ethanol solutions followed by acetone and embedded in epoxy resin. For T-tubule staining, small fiber bundles were fixed in $3.5 \%$ glutaraldehyde in $0.1 \mathrm{M}$ sodium-cacodylate buffer $\left(\mathrm{pH} 7.2,4^{\circ} \mathrm{C}\right)$ containing $75 \mathrm{mM} \mathrm{CaCl} 2$ for $1-2 \mathrm{~h}$. These specimens were then post-fixed in $2 \%$ OsO4- $0,8 \% \mathrm{~K} 3 \mathrm{Fe}(\mathrm{CN}) 6$ mixture for $1-2 \mathrm{~h}$ followed by rinse with $0.1 \mathrm{M}$ sodium cacodylate buffer with $75 \mathrm{mM} \mathrm{CaCl} 2(49,50)$. The specimens were rapidly dehydrated in graded ethanol and acetone, infiltrated with Epon-acetone (1:1) mixture for $2 \mathrm{~h}$, and embedded in Epon. Ultrathin sections (about 30-40 nm) were cut in Leica Ultracut R microtome (Leica Microsystem, Austria) using a Diatome diamond knife (DiatomeLtd. CH-2501 Biel, Switzerland) and stained in $4 \%$ uranyl acetate and lead citrate. Sections were examined with a FP 505 Morgagni Series 268D electron microscope (Philips), equipped with Megaview III digital camera and Soft Imaging System (Germany).

2.6.1 Classification of muscle fibers.

Muscle fibers were qualitatively analysed, observing every single fiber at low, intermediate, and high magnification The fibers were classified according to the following parameters: a) general shape of the fiber: large, straight, and cylindrical vs. small, wavy and variable in diameter, the latter being signs of denervation; b) presence of denervation features such as degeneration of the myofibrillar structure, thickening of the $\mathrm{Z}$ lines, nuclear and mitochondria grouping, etc.; c) general appearance of the myoplasm taking into consideration the presence of areas with a clearly visible I-A band striation. In the denervated patients (Table I), the analysed fibers (column A) were classified depending on the presence or absence of denervation traits (column B). Among this last group, 


\section{Muscle atrophy due to SCI can be reversed in complete absence of peripheral nerves}

European Journal Translational Myology - Basic Applied Myology 2012; 22 (4): 161-200

the number of fibers having some striated areas (column C) was counted. On the other hand, in the FES treated patients, (Table II), the fibers were classified depending on whether or not they were still atrophic/degenerated (columns B), or they presented instead obvious signs of recovery, such as a larger diameter and clear I-A striation in the majority of the fiber interior (column C). Among the recovering fibers, the number of fibers having regions in which the myofibrillar structure was not completely re-organized was counted (column D).

In the SCI group that had not undergone FES treatment, we analysed five patients (27 to 37 years of age) with denervation times ranging from 11 months to 4 years (see Table I), while, the FES treated group, patients ( 30 to 58 years of age) had been denervated for 3.6 years or longer (up to 10,6 years). This latter group had been treated with FES for varying periods of time (see Table II). FES training was started anywhere from 1.2 to 2.0 years after injury. This process of classification was performed over the entire visible fiber interior for each individual fiber before placing it in one of the categories included in Table I and II.

2.6.2 Size distribution spectrum of total myofibers

Semi-thin sections $(1 \mu \mathrm{m})$, stained using conventional techniques with toluidine blue (from Merck, Darmstadt, Germany), were used to plot fiber size distribution. The minimum transverse diameter of each myofiber was measured against a reference ruler. The myofibers were grouped and relative percentages plotted in $10 \mu \mathrm{m}$ steps (32).

2.6.3 Morphometric analysis

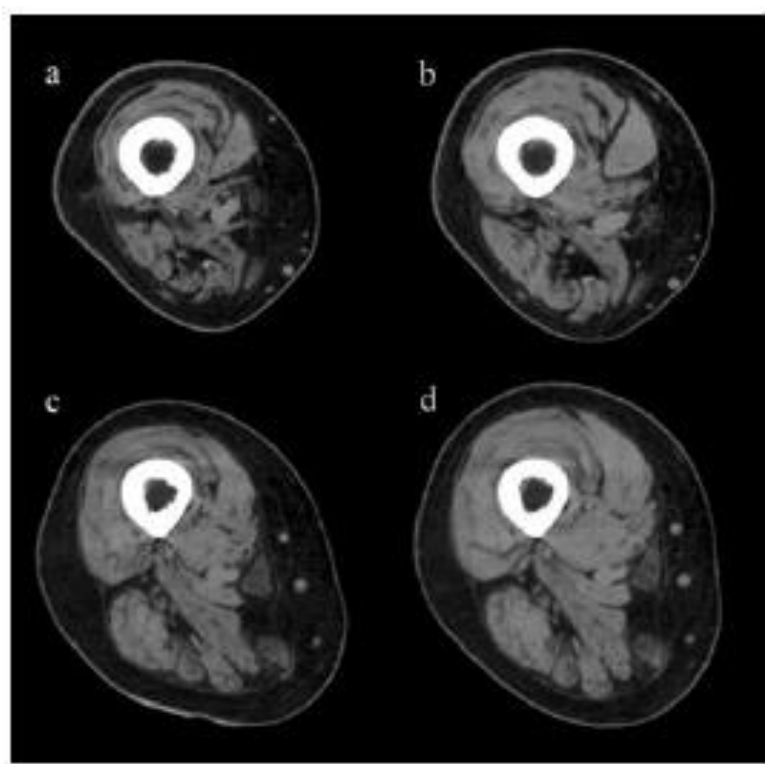

Fig 20 CT scans of the thigh muscles. CT scans of the right thighs $20 \mathrm{~cm}$ below trochanter major: (a) $>10$ years denervated; (b) same subject as (a), 1 year stimulated; (c) 1.7 years denervated; (d) same subject as (c), 1 year stimulated.

Images were acquired using a Zeiss microscope connected to a Leica DC $300 \mathrm{~F}$ camera at low magnitude under the same conditions used to acquire a reference ruler. Morphometric analysis was performed with Scion Image for Windows version Beta 4.0.2 (by 2000 Scion Corporation), free software downloaded from the web site: www.scioncorp.com.

Table I. Cross sectional area and density of CT-scan Quadriceps

\begin{tabular}{cccccccc}
\hline PAT Nr. & \multicolumn{3}{c}{ CROSS SECTIONAL AREA } & \multicolumn{3}{c}{ DENSITY } \\
& BEFORE & AFTER & $\mathrm{cm}^{2}$ & $\%$ & BEFORE & AFTER & DIFF.HU \\
\hline 1 & 24,51 & 38,04 & 13,53 & 55,20 & 15,30 & 30,50 & 15,20 \\
2 & 44,13 & 68,74 & 24,61 & 55,77 & 50,80 & 52,00 & 1,20 \\
3 & 24,56 & 36,01 & 11,45 & 46,62 & 13,30 & 24,20 & 10,90 \\
4 & 45,45 & 55,60 & 10,15 & 22,33 & 17,30 & 25,90 & 8,60 \\
5 & 15,76 & 19,42 & 3,66 & 23,22 & 16,30 & 17,47 & 1,17 \\
6 & 45,52 & 56,27 & 10,75 & 23,61 & 12,70 & 22,60 & 9,90 \\
7 & 35,30 & 43,70 & 8,40 & 23,70 & 27,10 & 18,05 & $-9,05$ \\
8 & 37,30 & 41,60 & 4,30 & 11,50 & 26,70 & 21,54 & $-5,16$ \\
9 & 82,19 & 91,46 & 9,27 & 11,27 & 52,26 & 55,01 & 2,75 \\
10 & 55,08 & 65,36 & 10,28 & 18,66 & 50,80 & 53,01 & 2,21 \\
11 & 40,33 & 46,99 & 6,66 & 16,51 & 35,20 & 41,13 & 5,93 \\
12 & 37,91 & 43,84 & 5,93 & 16,51 & 37,11 & 42,90 & 5,79 \\
13 & 18,36 & 20,28 & 1,92 & 10,46 & 16,00 & 16,51 & 0,51 \\
14 & 40,08 & 55,52 & 15,44 & 38,52 & 50,60 & 50,40 & $-0,20$ \\
mean & 39,03 & 48,77 & 9,74 & 26,71 & 30,11 & 33,66 & 3,55 \\
SD & 16,73 & 19,02 & 5,73 & 15,81 & 15,74 & 14,72 & 6,43 \\
\hline \hline
\end{tabular}


Muscle atrophy due to SCI can be reversed in complete absence of peripheral nerves

European Journal Translational Myology - Basic Applied Myology 2012; 22 (4): 161-200

Table II. Cross sectional area and density of CT-scan of Hamstrings.

\begin{tabular}{cccccccc}
\hline PAT Nr. & \multicolumn{3}{c}{ CROSS SECTIONAL AREA } & \multicolumn{3}{c}{ DENSITY } \\
& BEFORE & AFTER & DIFF. $\mathrm{cm}^{2}$ & DIFF. $\%$ & BEFORE & AFTER & DIFF.HU \\
\hline 1 & 20,11 & 29,29 & 9,18 & 45,64 & $-4,10$ & 14,50 & 18,60 \\
2 & 35,91 & 38,03 & 2,12 & 5,90 & 39,10 & 44,60 & 5,50 \\
3 & 20,64 & 27,41 & 6,77 & 32,80 & 0,70 & 16,40 & 15,70 \\
4 & 30,49 & 31,03 & 0,64 & 1,77 & $-3,40$ & 4,40 & 7,80 \\
5 & 7,09 & 9,57 & 2,48 & 34,90 & $-9,70$ & $-12,74$ & $-3,04$ \\
6 & 32,08 & 34,87 & 2,79 & 8,70 & $-0,50$ & 2,40 & 2,90 \\
7 & 35,96 & 42,54 & 6,58 & 18,30 & 25,90 & 15,76 & $-10,14$ \\
8 & 34,08 & 42,30 & 8,22 & 24,12 & 28,50 & 26,02 & $-2,48$ \\
9 & 55,34 & 54,51 & $-0,83$ & $-1,50$ & 46,42 & 47,26 & 0,84 \\
10 & 41,07 & 47,21 & 6,14 & 14,95 & 44,66 & 46,25 & 1,59 \\
11 & 32,13 & 38,37 & 6,24 & 19,42 & 15,43 & 25,93 & 10,50 \\
12 & 35,03 & 38,03 & 3,00 & 8,56 & 18,68 & 29,11 & 10,43 \\
13 & 12,79 & 10,10 & $-2,69$ & $-21,03$ & $-16,30$ & $-19,91$ & $-3,61$ \\
14 & 37,03 & 47,16 & 10,13 & 27,36 & 40,06 & 36,20 & 3,86 \\
mean & 30,70 & 35,03 & 4,34 & 15,71 & 16,10 & 19,73 & 4,18 \\
SD & 12,20 & 12,98 & 3,85 & 17,14 & 21,68 & 21,00 & 7,92 \\
\hline \hline
\end{tabular}

\subsection{PREPARATION OF FIGURES.}

Figures were mounted and labeled using Adobe Photoshop® v7.0.

\section{RESULTS}

\subsection{CLINICAL OBSERVATIONS}

\subsubsection{Force measurements}

Before FES training tight muscles of long-term DDM patients are unable to generate a measurable force. Indeed, this feature is one of the most valuable functional markers of DDM in SCI patients. After 4-6 months of stimulation with single twitches, when muscle excitability improves enough to allow tetanic contractions, the first measurement of muscle contraction force is performed. Unfortunately, followup of muscle performance are sometimes disturbed in these patients, due to unwanted simultaneous stimulation of antagonistic muscles (i.e., hamstrings) due to high voltage stimulation. In almost all paraplegic patients, the knee extension torque does not correlate with muscle tension and muscle mass. Anyhow, during the first 12 months of FES training the

Table III Atrophy and Distrophy of human Long-Term Denervated muscle

\begin{tabular}{|c|c|c|c|c|c|c|}
\hline \multirow{2}{*}{\multicolumn{2}{|c|}{ Time }} & \multirow{3}{*}{$\frac{\text { Minimum diameter }}{\mu \mathrm{m}}$} & \multicolumn{4}{|c|}{ Percent Area covered by } \\
\hline & & & \multirow[b]{2}{*}{ Myofibers } & \multirow[b]{2}{*}{ Adipocytes } & \multirow[b]{2}{*}{ Collagen } & \multirow{2}{*}{$\begin{array}{c}\text { Loose } \\
\text { connective tissue }\end{array}$} \\
\hline Denervation & FES & & & & & \\
\hline 0.7 & - & $18.1 \pm 9.9$ & 49.3 & 2.7 & 19.5 & 28.5 \\
\hline 0.7 & - & $18.6 \pm 7.4$ & 52.6 & 2.5 & 6.3 & 38.6 \\
\hline 0.7 & - & $20.7 \pm 16.8$ & 64.0 & 6.3 & 2.3 & 27.4 \\
\hline 2.9 & - & $15.9 \pm 10.3$ & 26.4 & 12.6 & 54.2 & 6.8 \\
\hline 2.9 & - & $22.3 \pm 18.0$ & 11.6 & 3.1 & 18.2 & 67.1 \\
\hline 3.3 & - & $8.12 \pm 4.5$ & 5.4 & 44.8 & 19.1 & 30.7 \\
\hline 3.5 & - & $20.8 \pm 19.4$ & 16.0 & 14.8 & 38.9 & 30.3 \\
\hline 3.5 & - & $20.4 \pm 14.0$ & 12.1 & 6.2 & 9.0 & 72.6 \\
\hline 4.0 & - & $9.0 \pm 10.5$ & 14.4 & 9.2 & 8.1 & 68.2 \\
\hline 8.6 & - & $8.4 \pm 3.7$ & 2.0 & 14.6 & 3.3 & 80.1 \\
\hline 8.7 & - & $4.5 \pm 1.5$ & 1.2 & 30.5 & 65.3 & 3.0 \\
\hline 8.7 & - & $7.7 \pm 3.7$ & 2.4 & 35.0 & 50.0 & 12.6 \\
\hline 19.0 & - & $15.3 \pm 15.3$ & 28.4 & 2.6 & 15.0 & 54.0 \\
\hline 19.0 & - & $21.6 \pm 25.3$ & 22.5 & 25.5 & 13.5 & 38.5 \\
\hline Mean & & 14.9 & 25.7 & 12.8 & 18.4 & 43.2 \\
\hline$\pm \mathrm{SD}$ & & 6.0 & 23.7 & 12.1 & 18.7 & 24.8 \\
\hline
\end{tabular}


Muscle atrophy due to SCI can be reversed in complete absence of peripheral nerves

European Journal Translational Myology - Basic Applied Myology 2012; 22 (4): 161-200
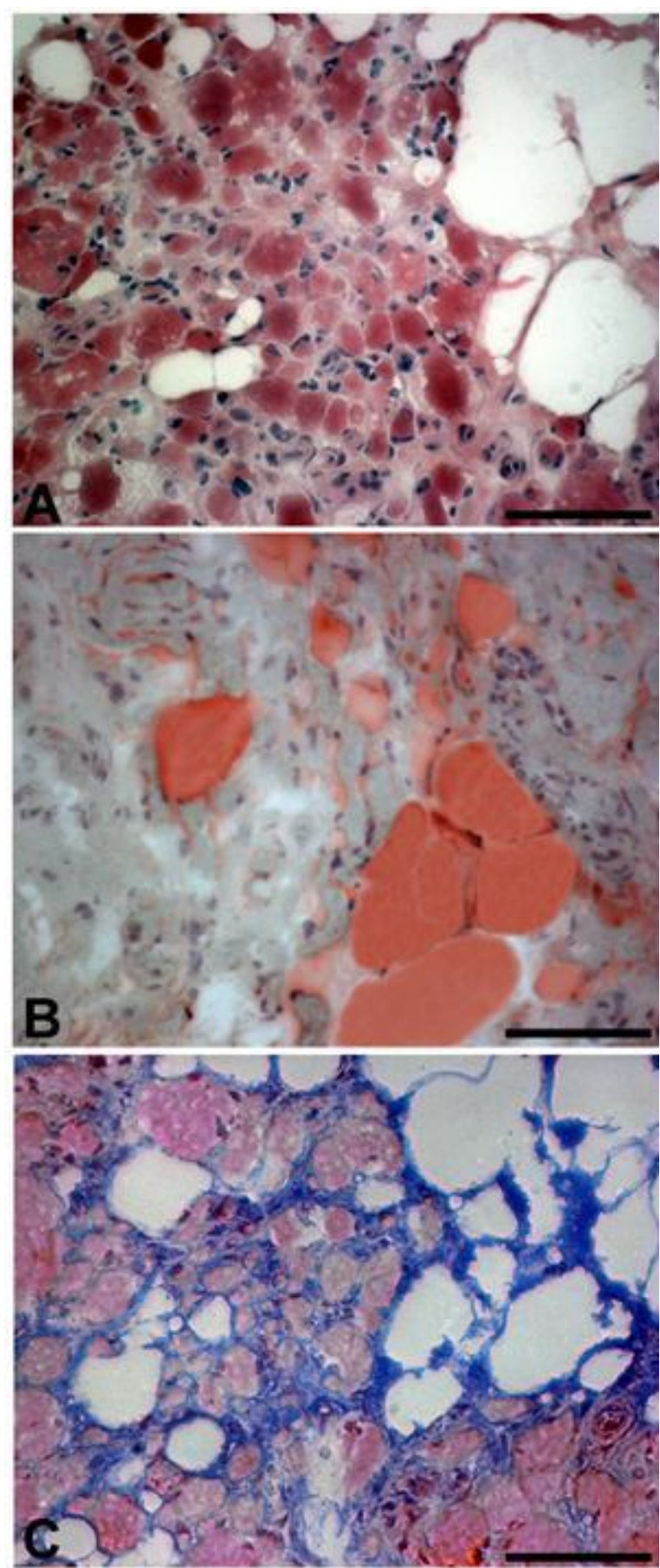

Fig 21 Human long-term denerveted quadriceps femoris: Light microscopy. A) $H \& E$ stain. B) oil red $O$ stain for lipid distribution. C) The Mallory Trichrome stain for demonstration of distribution of collagen. Scale Bar: $100 \mu \mathrm{m}$.

mean isometric knee extension torque (measured in sitting position with $90^{\circ}$ knee flexion) increases of 7,6 $\pm 25,2 \mathrm{Nm}$ above the first measurable value. The knee extension torque of DDM patients is at the beginning of training in a range of $0-5 \%$ and increases up to about $20 \%$ of maximum torque developed by healthy

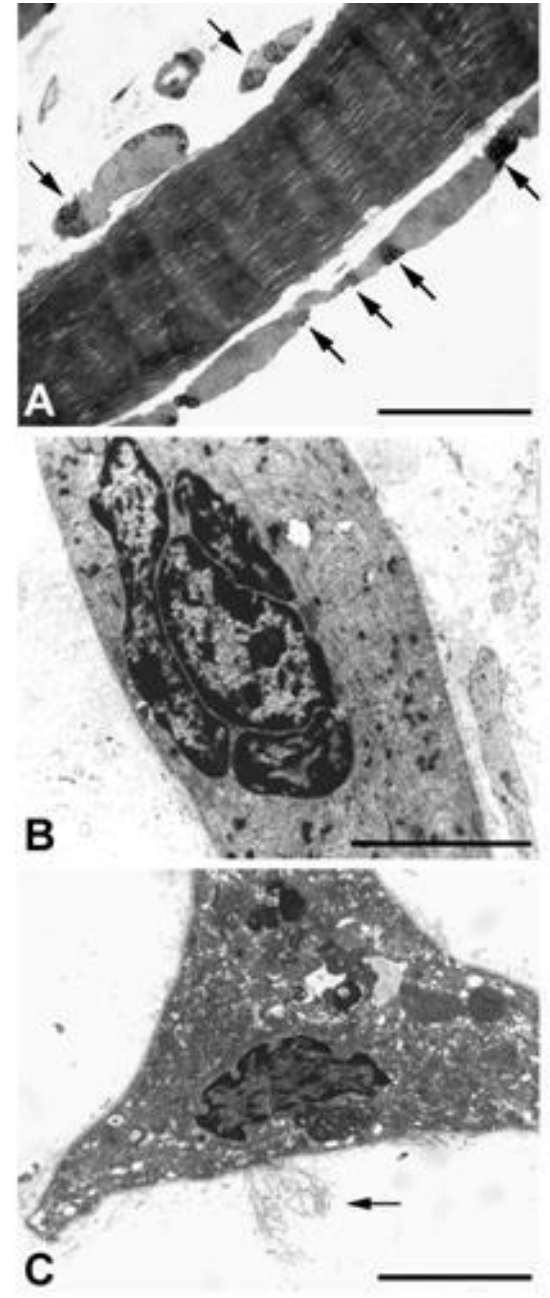

Fig 22 Ultrastructural feature of severe atrophy in long-term denervated myofibers. A) longitudinal semi-thin section of severely atrophic myofibers: arrows point to clumps of myonuclei, which alternate with long stretches of anucleated, amyofibrillar sarcoplasm. Scale bar $50 \mu \mathrm{m}$. B) electron micrograph of a longitudinal very small myofiber showing a clump of four myonuclei: myofibrillar structure and mitochondria are scarce or absent. Scale bar: $10 \mu \mathrm{m}$. C) cross-sections of a very small myofiber: the angulated profile and the overabundant folded layers of the original basal lamina (arrow) are also peculiar features of severe atrophy. Scale bar: $10 \mu \mathrm{m}$.

persons (209 Nm) [73]. The high standard deviation is caused by the unwanted co-contractions of the hamstrings due to high voltage stimulation, which is related to both length of denervation and thickness of subcutaneous fat acting as an electrical shunt. 3.1.2 CT-cross sectional area and muscle density 


\section{Muscle atrophy due to SCI can be reversed in complete absence of peripheral nerves}

European Journal Translational Myology - Basic Applied Myology 2012; 22 (4): 161-200

Measurement of muscle cross sectional area of the thigh by means of CT-scans revealed the enormous

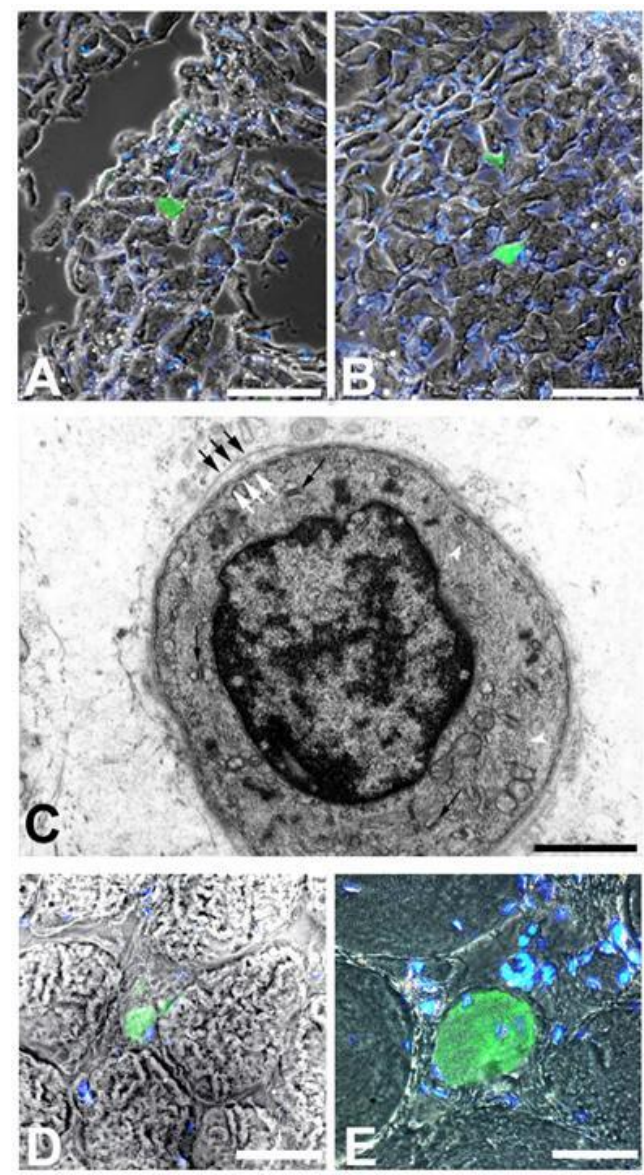

Fig 23 Regenerative events in long-term denervated muscle without and with FES: immunohistochemistry and electron microscopy. A, 0.7-year denervation and B, 8.7-year denervation. Early regenerated myofibers stained with antibody antiMHCemb are in green. Scale bar: 50 um. C, E.M. regenerating myotube in long term denervated muscle surrounded by two layers of basal lamina: black and white arrows points to old and new one. The small fiber presents a central nucleus, few thick filaments (white arrowheads), mitochondria, and triads (thin black arrows). $A$ and $B$, scale bar: $100 \mu \mathrm{m}$. C, scale bar: $2.0 \mu \mathrm{m} . \mathrm{D}$, 2-year denervation followed by 4.3-year FES training and $E$, 2.3-year denervation followed by 2.7-year FES training. Early regenerated myofibers are in green. Double exposure anti-MHCemb/Hoechst 33258 shows that some blue nuclei are centrally located. The myofibers are much larger that in panels $A$ and $B$, scale bar: $50 \mu \mathrm{m}$. amount of atrophy of the denervated muscles (Figure $20)$. The muscle area is reduced to $40 \%$ of the initial state after 1-2 years of denervation73. Moreover the decrease in muscle cross sectional area is accompanied by structural degeneration in muscle histology and by infiltration of fat tissue among muscles (as recognized in the CT-scan). In our patients the mean cross sectional area of the m. quadriceps measured $20 \mathrm{~cm}$ below the trochanter tops was $39,03 \mathrm{~cm} 2$ at the beginning of the stimulation program and $48,77 \mathrm{~cm} 2$ after 1 year of stimulation. That equals to an increase of muscle area of $9,78 \mathrm{~cm} 2$ or $26,65 \%$ (Table I) Notice that also the cross sectional area of the co-stimulated thigh flexors increased by stimulation of the skin over the quadriceps femoris muscle (Table II). The mean density of the $\mathrm{m}$. quadriceps measured in Houndsfield Units was $30.1 \mathrm{HU}$ at the beginning of the stimulation program and $33.67 \mathrm{HU}$ after 1 year of stimulation, that is, an increase of $3.55 \mathrm{HU}$ muscle density or $11.86 \%$ (Table I).

3.2 LIGHT AND ELECTRON MICROSCOPY: LONGTERM DENERVATED MUSCLE

3.2.1 Atrophy and dystrophy of human long-term denervated muscle.

Denervation of skeletal muscle causes rapid loss in both mass and contractile force, which is followed long-term by other severe structural changes (lipodystrophy). Figure 21 shows three images of a representative 8.7-year denervated human muscle after staining with $\mathrm{H}-\mathrm{E}$ (panel $\mathrm{A}$ ), oil red $\mathrm{O}$ stain for lipid distribution (panel B) and the Mallory trichrome stain for outlining the distribution of collagen (panel $\mathrm{C}$ ). This is a typical long-term denervated human muscle, in which the atrophic myofibers have been substituted by adipocytes and collagen. Table III shows the relative percentages of myofiber area, adipocytes and collagen in the biopsies from long-term denervated subjects. Adipocytes and collagen, which are scarce in humans for up to a year of lower motoneuron

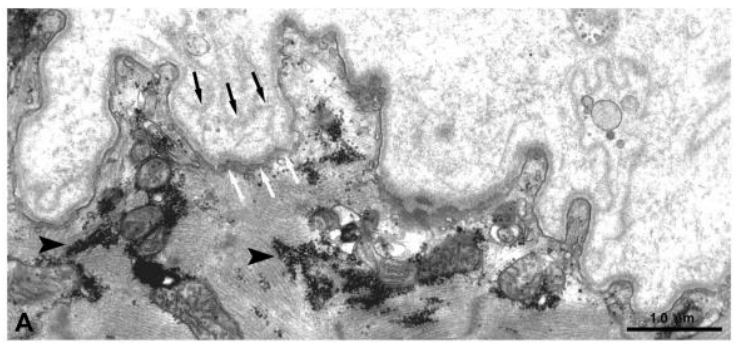

Fig 24 Myofiber Regeneration. Portion of fiber showing double-layered basement membrane (see black and white arrows) that surrounds this relatively healthy myofiber. This fiber is identified as new fiber (regenerated during the month before biopsy) undergoing atrophy and degeneration. 
Muscle atrophy due to SCI can be reversed in complete absence of peripheral nerves

European Journal Translational Myology - Basic Applied Myology 2012; 22 (4): 161-200

\section{Table II. Effects of long-term denervation on skeletal muscle fibers.}

All the fibers analysed presented evident signs of disorganization (column B, see also Errore.

$L$ 'origine riferimento non è stata trovata.), even in those patients with shorter denervation times (SCI 1 and 2). In column $C$ the number (and percentage) of fibers that still present few areas with a clear I-A band striation is reported.

\begin{tabular}{cccccc}
\multicolumn{2}{c}{ SCI patients } & A & B & C \\
\hline $\begin{array}{c}\text { Patien } \\
\text { ts }\end{array}$ & $\begin{array}{c}\text { Age at the } \\
\text { Time of Biopsy }\end{array}$ & $\begin{array}{c}\text { Time intervals between } \\
\text { SCI and muscle biopsy }\end{array}$ & $\begin{array}{c}\text { n. of } \\
\text { fibers } \\
\text { analysed }\end{array}$ & $\begin{array}{c}\text { n. of } \\
\text { atrophying } \\
\text { fibers }\end{array}$ & $\begin{array}{c}\text { Fibers with } \\
\text { striated areas }\end{array}$ \\
\hline SCI 1 & 30 year & 11 months & 38 & $38(100 \%)$ & $8(21 \%)$ \\
SCI 2 & 37 year & 1,3 years & 41 & $41(100 \%)$ & $0(0 \%)$ \\
SCI 3 & 37 year & 1,8 years & 47 & $47(100 \%)$ & $2(4 \%)$ \\
SCI 4 & 27 year & 1,9 years & 38 & $38(100 \%)$ & $5(13 \%)$ \\
SCI 5 & 26 year & 4,0 years & 49 & $49(100 \%)$ & $0(0 \%)$
\end{tabular}

denervation, increase thereafter, in spite of the fact that the mean fiber diameter of the measurable myofibers is less that $20 \mu \mathrm{m}$ from 0.7 to 19.0 years of denervation (mean diameter $14.9+/-6.0$ in 20 biopsies). Adipocytes begin to accumulate one to two years after denervation, constituting up to $30 \%$ of muscle biopsy area after 8 10 years of continued denervation (denervated, degenerated muscle, DDM).

3.2.2 Severely atrophic myofibers: nuclear clumping and disorganization of the myofibrillar
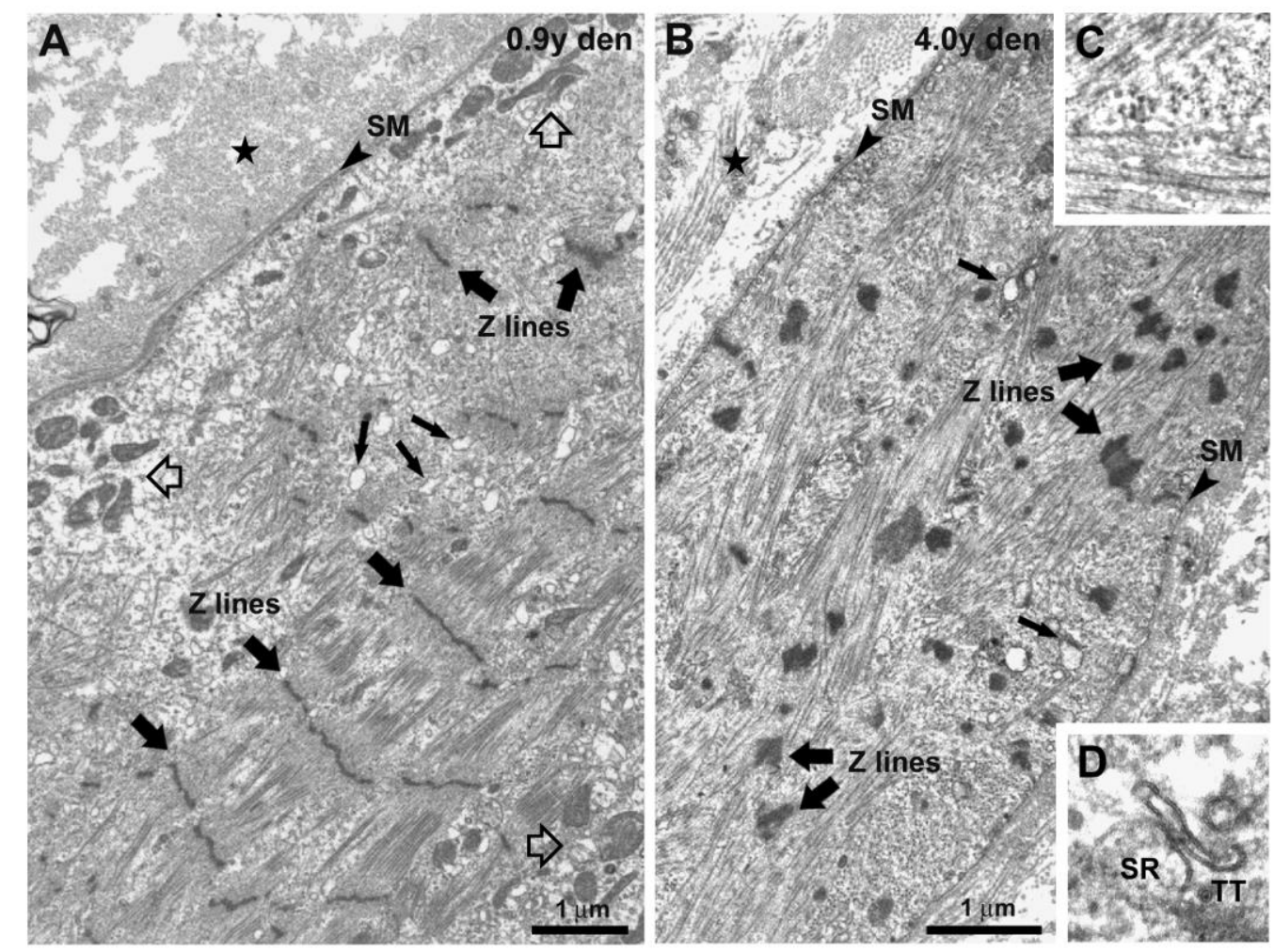

Fig 25 Effects of long-term denervation on skeletal muscle fibers. A, SCI 1 patient (11 months of denervation): denervation-induced progressive disarrangement of the internal structure of muscle fibers usually starts at the fiber periphery (SM, surface membrane) and involves contractile, EC coupling, and mitochondrial apparatuses. B, SCI 5 patient (4 years of denervation): long-lasting denervation determines severe atrophy and complete disruption of the internal organization of the fibers. Panels $C$ and $D$ show respectively an area with miss oriented contractile filaments and an abnormal calcium release unit. 
Muscle atrophy due to SCI can be reversed in complete absence of peripheral nerves

European Journal Translational Myology - Basic Applied Myology 2012; 22 (4): 161-200

components.

Long-term denervated fibers undergo a progressive disorganization of both the contractile and ECC coupling apparatuses. Figure 22 shows severely atrophic myofibers of muscle that has been denervated for 15 months, which display several peculiar features
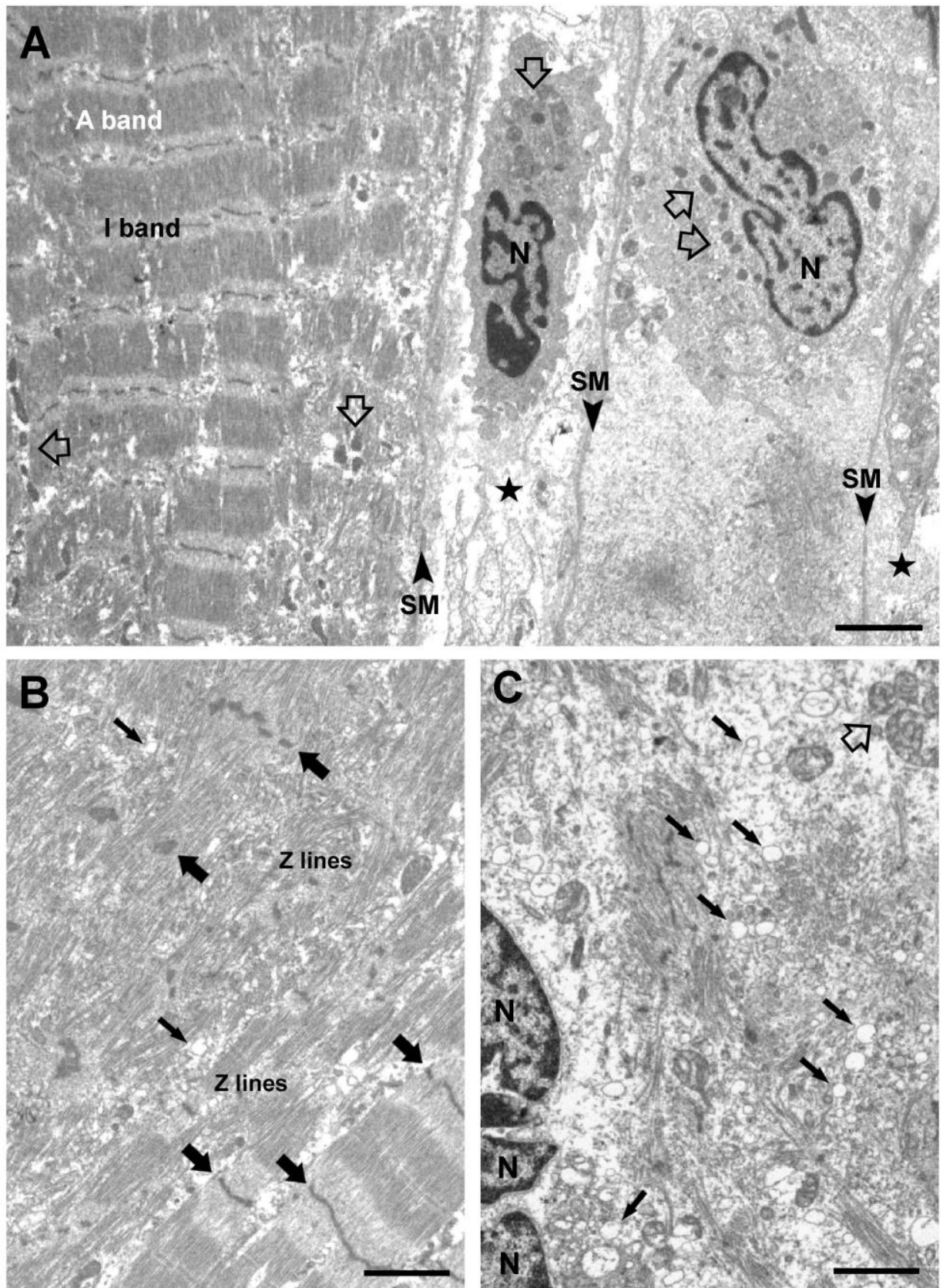

Fig 26 Ten months denervated muscle biopsy. Electron microscopy made possible a more detailed observation of the ultrastructure of the denervated skeletal muscle fiber, particularly of the myofibrils. Panel A shows a portion of a longitudinal section of a denervated muscle area. As confirm of the histological measurements, fibers shows an irregular distribution of the dimension than in normal muscle. In fact there are fibers (look at the left fiber of Panel A that preserve a quite extensive superficial area. In this fiber we found an area in which myofibrils have a normal ultrastructure even if these areas are usually very small and incomplete (Panel B for enlargement). Note that the contractile material generally disarranged or disappeared at the periphery of the fiber. In the same area there is another fiber, completely disarranged (look at the right of Panel A). Between the two major fibers there is a severely atrophic fiber. Nuclei seem to be more numerous (Panel C) and often have a central location. 
Muscle atrophy due to SCI can be reversed in complete absence of peripheral nerves

European Journal Translational Myology - Basic Applied Myology 2012; 22 (4): 161-200
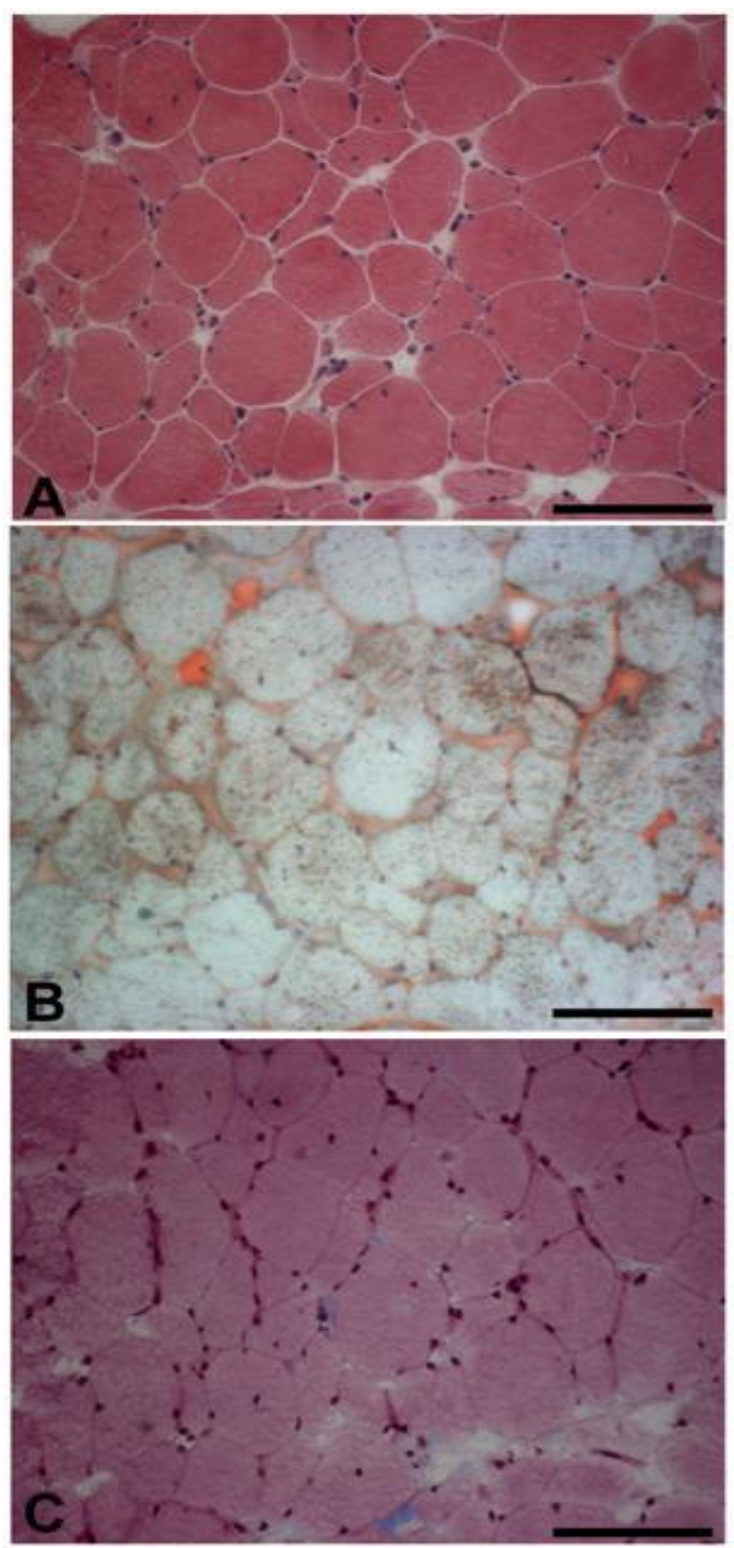

Fig 27 Effects of FES training on histology of human long-term denervated muscle. Light microscopy. A, $H$-E stain. B, oil red $O$ stain for lipid distribution and $C$, the Mallory trichrome stain for demonstration of distribution of collagen. The biopsy shows many large round fibers even if a residual population of small myofibers is still present among the large ones. The effect of FES training on long-term denervated muscle is striking: compare Figure 27 with Figure 21. Adipocytes are absent, collagen is of almost normal appearance. Scale bars: $100 \mu \mathrm{m}$.

of long-term denervation. One characteristic is the presence of clumps of myonuclei (Figs. 22 A and B) alternating with long stretches of anucleated sarcoplasm, in which myofibrils are absent. Figure 22

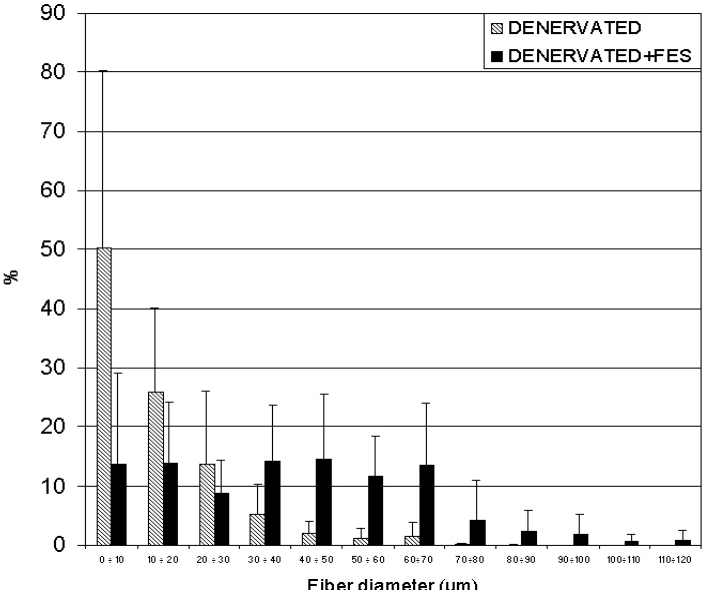

Fig 28 Fiber size spectrum of long-term denervated muscle vs. FES training. The cumulative value of fiber sizes in twenty muscle biopsies after either long-term denervation (grey bars), or FES treatment (black bars). More than $50 \%$ of myofibers have diameter smaller than $10 \mu \mathrm{m}$ in DDM biopsies, while the FES trained subjects have more that 50\% of myofibers larger than $30 \mu \mathrm{m}$

C shows a cross section of a severely atrophic myofiber, displaying an unusual triangular shape. In addition to the angulated aspect, the presence of overabundant folded layers of the original basal lamina (arrow) is also a peculiar feature of severe atrophy. The majority of the myofibers, either round or angulated, with central or peripheral clumps of myonuclei, is also often surrounded by an increased numbers of collagen sheets and exhibits either a disorganization or a full loss of myofibrils. These myofibers also tend to have lipid bodies, folds of the basement membrane, and sparse mitochondria.

\subsubsection{Myofibers regeneration in Long-Term Denervated Muscle.}

In long-term denervated muscles, contrary to the general expectation, myofiber regeneration is frequently observed, even if at a low rate. In Figure 23 A-B, transverse sections of long-term denervated human muscle biopsies are shown displaying green The round, green areas seen representing cross sections of myofibers positive for the anti MHC-emb antibody. These are newly regenerated myofibers, which had developed during the last couple of weeks before biopsy. From 9-month, up to 19.0-year denervation, the recently regenerated myofibers represent $1.1 \%+/-$ $1.2 \%$ of total myofiber population, with any timedependent variability.

Electron microscopy confirms the presence of myotubes in long-term denervated human muscle. Figure $23 \mathrm{C}$ and Figure 24 show that a double-layer of basement membrane (black and white arrowheads) 
Muscle atrophy due to SCI can be reversed in complete absence of peripheral nerves

European Journal Translational Myology - Basic Applied Myology 2012; 22 (4): 161-200

Table V. FES of Long-Term Denervated Human Muscle: Reversion of Distrophic Changes.

\begin{tabular}{|c|c|c|c|c|c|}
\hline & & & & ent area covs & \\
\hline & & Minimum diameter & & & Colleuren and loove \\
\hline Denervation & FES & $\mu \mathrm{m}$ & Myotibers & Adipocytes & connective tissue \\
\hline $1.8+$ & 2.2 & $43.3 \pm 12.3$ & 95.1 & 2.7 & 2.2 \\
\hline $1.8+$ & 2.2 & $35.8 \pm 17.0$ & 96.8 & 1.6 & 1.6 \\
\hline $2.9+$ & 2.2 & $31.4 \pm 16.7$ & 98.5 & 1.1 & 0.4 \\
\hline $2.9+$ & 2.2 & $34.7 \pm 16.1$ & 97.6 & 1.0 & 1.4 \\
\hline $1.3+$ & 2.4 & $30.7 \pm 23.7$ & 97.5 & 13 & 1.2 \\
\hline $1.3+$ & 2.4 & $26.7 \pm 22.9$ & 96.9 & 3.0 & 0.1 \\
\hline $23,0+$ & 2.8 & $36.7 \pm 42.3$ & 79.9 & 11.0 & 9.1 \\
\hline $23.0+$ & 2.8 & $24.9 \pm 29.0$ & 94.3 & 1.8 & 3.9 \\
\hline $0.9+$ & 3.4 & $36.3 \pm 29.1$ & 93.3 & 3.7 & 3.0 \\
\hline $0.9+$ & 3.4 & $25.4 \pm 27.0$ & 95.7 & 3.1 & 1.2 \\
\hline $2.0+$ & 4.3 & $45.7 \pm 18.3$ & 97.5 & 0.2 & 2.3 \\
\hline $2.0+$ & 4.3 & $46.5 \pm 14.2$ & 98.0 & 0.5 & 1.5 \\
\hline $8.6+$ & 4.8 & $58.2 \pm 24.1$ & 96.3 & 1.2 & 2.5 \\
\hline $24.0+$ & 5.8 & $69.2 \pm 30.5$ & 79.3 & 1.9 & 18.8 \\
\hline $24,0+$ & 5.8 & $81.8 \pm 31.4$ & 87.1 & 1.2 & 11.7 \\
\hline $0.4+$ & 6.9 & $32.6 \pm 16.0$ & 95.2 & 1.8 & 3.0 \\
\hline $1.8+$ & 7.8 & $57.8 \pm 25.1$ & 99.0 & 0.5 & 0.5 \\
\hline $1.8+$ & 7.8 & $39.5 \pm 23.0$ & 92.9 & 3.9 & 3.2 \\
\hline $1.2+$ & 9.4 & $48.1 \pm 12.2$ & 97.7 & 0.5 & 1.8 \\
\hline $1.2+$ & 9.4 & $39.2 \pm 18.0$ & 97.4 & 0.5 & 2.1 \\
\hline Mean & & 42.2 & 94.3 & 2.1 & 3.6 \\
\hline$\pm \mathrm{SD}$ & & 14.8 & 5.7 & 2,4 & 4.6 \\
\hline p vs DDM & & $<0.0001$ & $<0.0 \times 001$ & $<0.001$ & $<0.001$ \\
\hline
\end{tabular}

delimits a very early regenerating myotube, which contains a few thick myofilaments.

The basal lamina double-layered fibers are rare, agreeing with the fact that anti-MHCemb antibodies stain about one percent of the myofibers in long-term denervated and degenerated human muscles.

\subsubsection{Ultrastructural analysis of long-term denervated muscle fibers}

The muscles of untreated patients show extreme features of classical denervation atrophy $[37,40,74]$, the severity of which, however, depends on the time elapsed from the injury. The histological analysis of some of these human biopsies have been already published in Kern et al. 2004 [72]. In each of the five biopsies at least 38 fibers were analyzed, (Table IV, column A) and classified depending on the presence and severity of denervation traits. All the fibers analyzed in each patient displayed classical denervation features such as disorganization of the contractile apparatus, thickening of the $\mathrm{Z}$ line, disarrangement of triads, mitochondria grouping, etc., including those fibers from patients with shorter denervation times (Table IV, column B). This semiquantitative classification of the fibers based on the degree of structural disarray revealed that already at 11 months of denervation very few areas with partially preserved cross striation remains and even those are lost with longer denervation periods (Table IV, column C).

At shorter times of denervation the disorganization of the fiber ultrastructure appear to be always more severe in the subsarcolemmal region, suggesting that the degeneration of the contractile apparatus must starts from the fiber periphery and proceed toward the fiber interior (Figures $25 \mathrm{~A}$ and 26). Myofibrils are usually small, often interrupted and/or completely missing from extended areas, and sarcomeres are often altered and with missing $\mathrm{M}$ lines (Figures 25). Widened and/or streaming $\mathrm{Z}$ lines, common feature also in a wide variety of muscle diseases, are frequent (Figure $25 \mathrm{~B}$ and 26) [40,74]. The widened intermyofibrillar spaces contain a not well differentiated cytoskeletal network with very little glycogen. The internal membrane systems of the SR are incomplete and vesiculated, while $\mathrm{T}$ tubules are hardly recognizable. Some associations of possible $\mathrm{T}$ tubules profiles with elements of the SR are present: they are usually very primitive looking and displays its altered morphology (Figure 25 D). Mitochondria are scarce, have no specific myofibril-related positioning, and often grouped in an abnormal fashion (Figure $25 \mathrm{~A}$, empty arrows). In longitudinal section, most fibers have variable diameter along the length and a wavy course, with the latter being probably caused by the fact that a well aligned fixation of fiber bundles is challenging in denervated muscle. Extracellular space between fibers is filled by a large amount of adipose-infiltrated connective tissue with heavy bundles of collagen fibers (Figure 25, asterisks). Denervation features are generally similar among different fibers and even different specimens. 

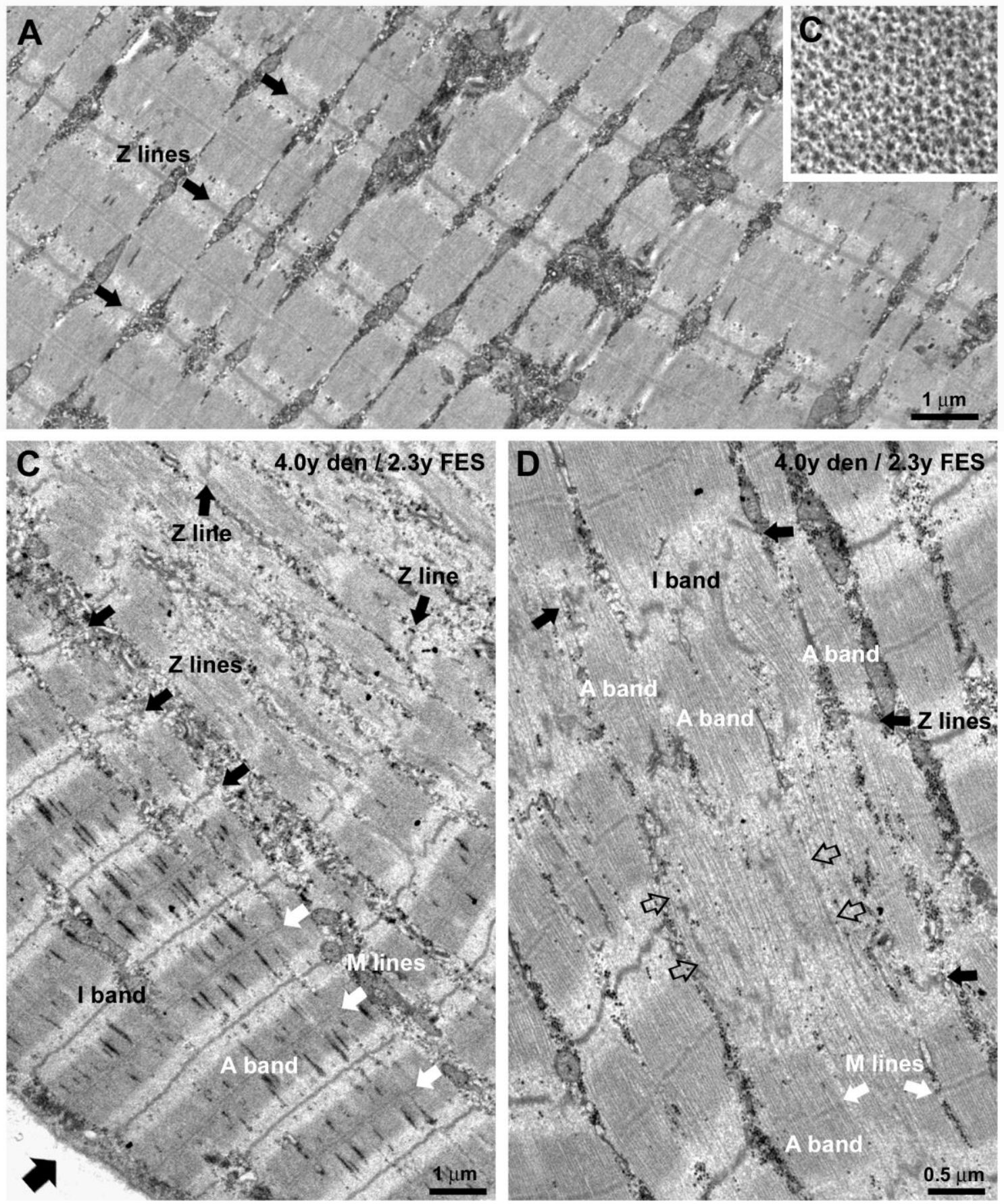

Fig 29FES-induced ultrastructural restoration of myofibrils. Restoration of the internal ultrastructure of skeletal fibers results in a transversal dark-pale band striation formed by fairly well aligned longitudinal myofibrils (panel A). In cross section, the zone of thick and thin filament overlap (A bands) of a restored myofibril shows the characteristic and extremely ordered hexagonal pattern of thick filaments (panel B). FES induced restoration of the contractile apparatus usually starts at the periphery of fiber (panel C, large arrow) and proceeds towards the interior as the fiber grows in size. The formation of nascent myofibrils involves an increase in the order of thin and thick filaments (panel D, empty arrows), the assemblage of primitive $A$ bands (labelled), and the formation and alignment of $Z$ lines (black arrows).

However, some discrimination should be made between patients with different denervation times. In fact, the longer is the denervation time, the higher is the degree of ultrastructural disorganization of the fiber 


\section{Muscle atrophy due to SCI can be reversed in complete absence of peripheral nerves}

European Journal Translational Myology - Basic Applied Myology 2012; 22 (4): 161-200

\section{Table III. FES treated patients.}

FES training results in a drastic reduction of severely atrophic fibers (column B), while most of them (85\% or more) present a re-organized contractile apparatus (column $C$, see also Fig 2). There is still a percentage of recovering fibers that present areas in which the myofibrils are not completely restored (column D, see also Fig. $2 \mathrm{~A}$ and B). The percentage of fibers in which a less complete core is detected decreases with increasing time of FES treatment (column D), indicates that either more fibers are fully recovered with the longer treatment, or that the fully recovered peripheral ring occupies a larger percentage of the total fiber volume.

\begin{tabular}{|c|c|c|c|c|c|c|c|c|}
\hline \multicolumn{2}{|l|}{ FES treated patients } & A & B & C & D \\
\hline $\begin{array}{c}\text { Patient } \\
s\end{array}$ & $\begin{array}{c}\text { Age at the } \\
\text { Time of Biopsy }\end{array}$ & $\begin{array}{c}\text { SCI and } \\
\text { FES }\end{array}$ & FES and biopsy & $\begin{array}{c}\text { SCI and muscle } \\
\text { biopsy }\end{array}$ & $\begin{array}{c}\text { Time intervals between: } \\
\text { analysed }\end{array}$ & $\begin{array}{c}\text { n. of } \\
\text { atrophic } \\
\text { fibers }\end{array}$ & $\begin{array}{c}\text { n. of } \\
\text { recoveredirecoverin } \\
\text { g fibers }\end{array}$ & $\begin{array}{c}\text { n. of partially } \\
\text { recovered } \\
\text { fibers }\end{array}$ \\
\hline FES 1 & 47 year & 1,2 years & 2,4 years & 3,6 years & 27 & $4(15 \%)$ & $23(85 \%)$ & $9(39 \%)$ \\
\hline FES 2 & 50 year & 1,7 years & 2,3 years & 4,0 years & 22 & $0(0 \%)$ & $22(100 \%)$ & $8(36 \%)$ \\
\hline FES 3 & 41 year & 2,0 years & 4,3 years & 6,3 years & 25 & $1(4 \%)$ & $24(96 \%)$ & $3(12 \%)$ \\
\hline FES 4 & 58 year & 1,9 years & 7,7 years & 9,6 years & 33 & $3(9 \%)$ & $30(91 \%)$ & $5(17 \%)$ \\
\hline FES 5 & 30 year & 1,3 years & 9,3 years & 10,6 years & 24 & $1(4 \%)$ & $23(96 \%)$ & $2(9 \%)$ \\
\hline
\end{tabular}

interior, and the higher is the accumulation of adiposeinfiltrated connective tissue in the extracellular space. Disrupted myofibrils and/or sarcomers are mostly found in those patients that have been denervated for shorted periods (patient SCI 1-4 and Fig. 26), while the longer the denervation period and the more difficult it gets to find sarcomereric structures. Mitochondria and $\mathrm{T}$ tubule/SR junctions are more frequent in specimens with shorter times of denervation, while are almost disappeared at the longer times

\subsection{LIGHT AND ELECTRON MICROSCOPY: ELECTROSTIMULATED MUSCLE}

\subsubsection{Effects of the FES Training on Long-Term} Denerveted muscle

Figure 27, shows the effects of 4.3-year electrostimulation training after 2.0-year DDM. Serial cryosections are stained with $\mathrm{H}-\mathrm{E}$ (panel A), oil red O stain for lipid distribution (panel B) and the Mallory trichrome stain for demonstration of distribution of collagen (panel C). The biopsy shows large round myofibers of heterogeneous size, but with large fibers clearly prevailing (panel A). Adipocytes are almost absent (Fig. 27, B) and collagen is present in normal amounts (Fig. 27, C). Table V confirms that in all the studied biopsies from FES trained subjects adipocytes are absent and collagen is present in normal amounts. Note that a residual population of small myofibers is present in the electro-stimulated muscles, as also shown by fiber size spectra (Figure 28). Panels D and E of Figure 23 confirm that a few of them, which stain positive by anti-MHC-emb antibodies, are the result of recent regeneration events

The mean value of the minimum diameter in the FES trained DDM is $42.2+/-14.8$ (SD) $\mu \mathrm{m}$. The data compare well with the values of $54.0+/-7.2 \mu \mathrm{m}$ for normal muscle in sedentary subjects and 14.9+/-6.0 (SD) $\mu \mathrm{m}$ for for long-term denervated human muscles, respectively (Table V).

In Figure 28 the cumulative myofiber spectrum of long-term DDM is compared to that after FES. Around $50 \%$ of myofibers have diameter smaller than $10 \mu \mathrm{m}$ in DDM biopsies, while the FES trained subjects have more that $50 \%$ of myofibers larger than $30 \mu \mathrm{m}$. Some FES myofibers look hypertrophic (diameter larger that $80 \mu \mathrm{m})$. Table $\mathrm{V}$, shows that the mean percentual myofiber area in FES biopsies is 94.3+/-5.7 SD ( $p<0.0001$ vs DDM 59.2+/37.8 SD), mean percentual fat area $2.1+/-2.4 \mathrm{SD}(\mathrm{p}<0.001$ vs DDM 12.8+/-12.1 $\mathrm{SD})$, mean percentual interstitial tissue area 3.6+/-4.6 SD ( $<<0.001$ vs DDM 61.6+/-20.1 SD). The mean percentual collagen area is in long-term denervated muscles $18.4+/-18.7$, while it is not easily recognizable in FES biopsies. These results are well within normal range and indicate that recovery of muscle trophism is indeed taking place.

\subsubsection{Ultra-structural analysis of FES trained denervated fibers.}

CT scan and hystological analysis have shown an extremely significant increase of muscle mass and fiber size respectively in the patients undergoing a FES treatment $[71,72]$. Denervation time and duration of FES treatment before biopsy for the five patients analysed in this study are shown in Table VI. Electron microscopy analysis of FES treated muscle fibers shows a striking restoration of the internal ultrastructure: this recovery results in a clear I-A band striation that is visible in the large majority of the thin sections area (Figure 29 A). In cross section, restored 

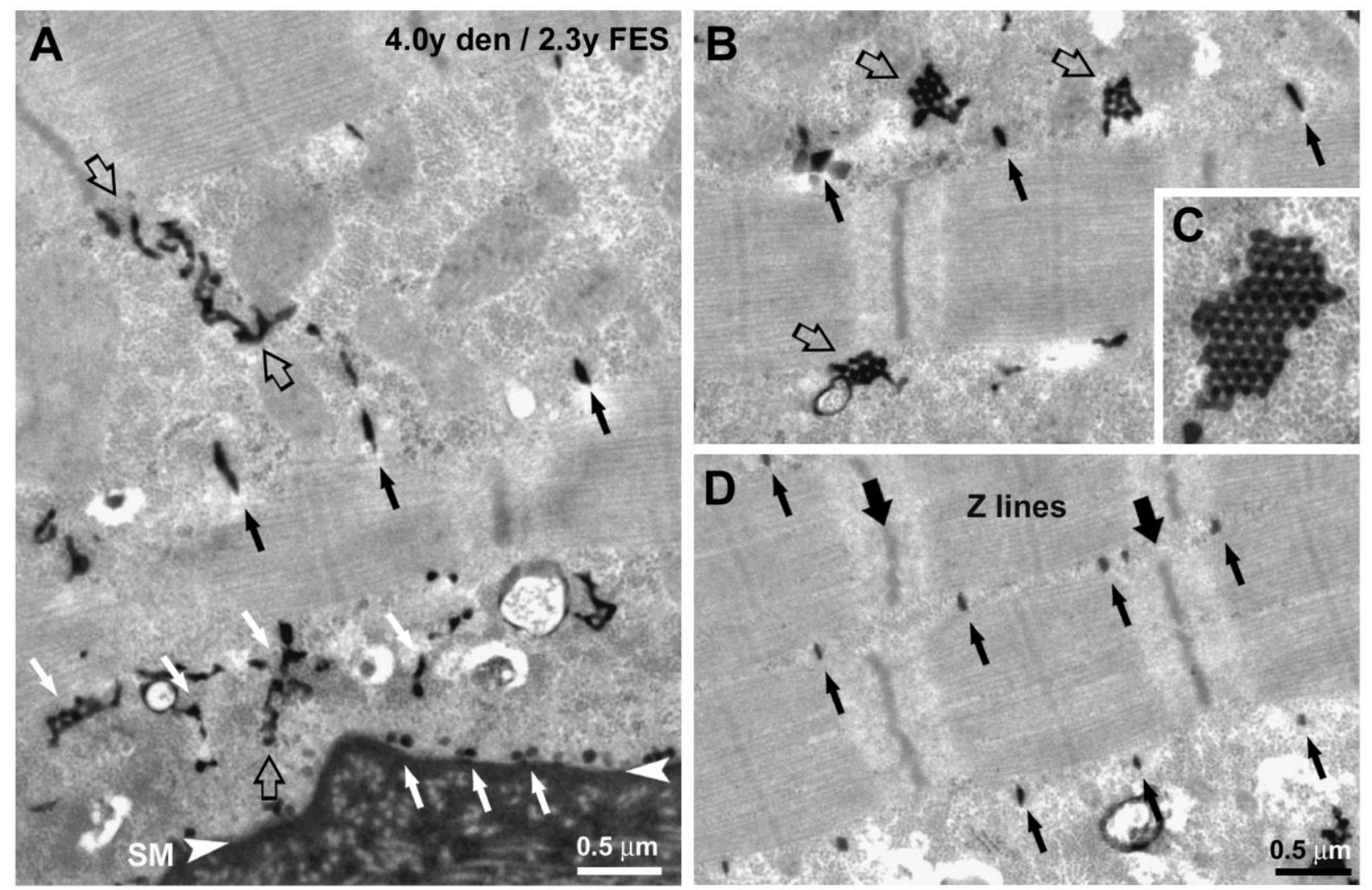

Fig 30 Formation of the T-tubule network in FES treated fibers. The development of T tubules in FES stimulated fibers involves the formation of multiple clusters of caveolae in the subsarcolemmal area (panel A, white arrows) that are continuous with tortuous $T$ tubules and labyrinthine structures (panel A and B, empty arrows). The network of maturing tubules becomes progressively transversal and better organized while it specifically associates to the I-A junction of mature myofibrils (small black arrows in all panels).

myofibrils show the characteristic and extremely ordered hexagonal pattern of thick filaments, indicating a general good reorganization of the sarcomeres (Figure $29 \mathrm{~B}$ ). The profiles of fibers in the FES treated patients were classified into three categories, shown in Table VI: 1) fibers that are still severely atrophic and that resemble fibers in nontreated patients (column B); 2) fibers that shows a dark and pale striation throughout the all segment visible in the thin section (see Figure $29 \mathrm{~A}$ ); and 3) fibers containing some regions in which myofibrils are not completely re-assembled, together will fairly well differentiated regions (column D, see Figure $29 \mathrm{C}$ and D). Column $\mathrm{C}$ in Table VI contains all fibers that appear either fully recovered or partially recovered.

The number of atrophic, or atrophying, fibers is drastically reduced following the FES training protocol: mostly less than $10 \%$, versus $100 \%$ in the untreated muscles (compare data in Table IV and Table VI). Note that the fibers in which the contractile apparatus appear as fully reorganized may have an inner core that is less disorganized but simply not present within the plane of the thin section. The percentage of fibers in which a less complete core is detected decreases with increasing time of FES treatment (column D). This indicates that either more fibers are fully recovered with the longer treatment, or that the fully recovered peripheral ring occupies a larger percentage of the total fiber volume.

The presence of containing internal cores in which myofibrils are not yet completely reassembled allowed us to observe that FES-induced restoration of the contractile apparatus always starts from the fiber periphery (Figure 29 C) and seems to proceeds towards the fiber interior as the fibers grow in size. Well organized myofibrils, with appropriately structured A and I bands form an ordered cross striation at the fiber's periphery (Figure 29 C), while myofibrils are sometimes less well organized in the fiber interior in which incomplete sarcomeres present a somewhat disordered striation and sometimes missing $\mathrm{Z}$ and $\mathrm{M}$ lines (Figure 29 D). The transition between more and less organized areas in fairly abrupt in some areas and more gradual in others, with transitional regions where $\mathrm{Z}$ lines are present, but are not yet straight (Figure $29 \mathrm{C}$ and D). Thick filaments are fairy well aligned but the edges of the A band are not well defined, the $\mathrm{M}$ line may be missing, and cross striation is not well 

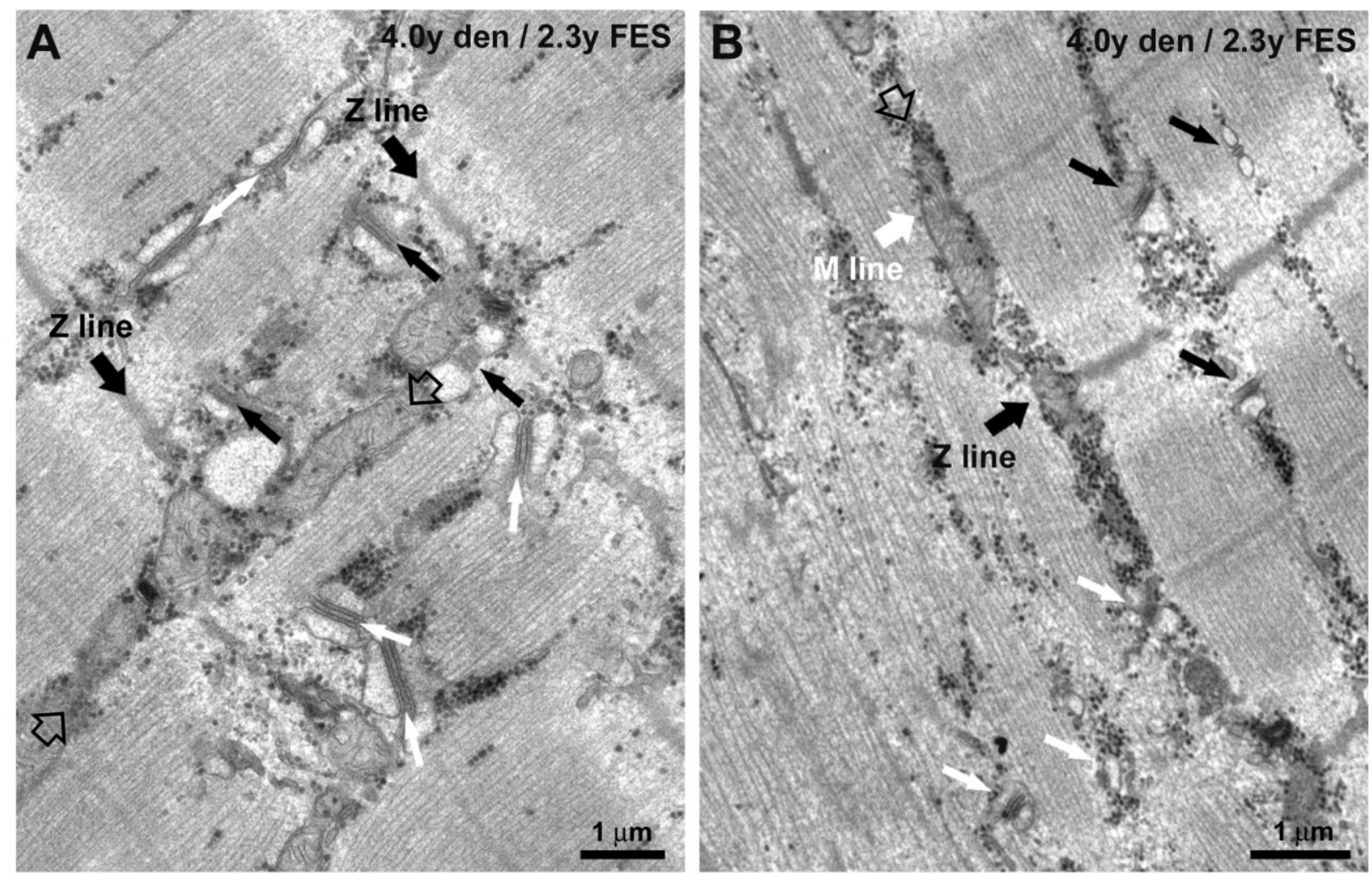

Fig 31 FES induced restoration of the EC coupling apparatus. The final positioning of triads at the sarcomere I-A junction characteristic of mammalian muscle in FES treated fibers is achieved by a gradual shift from an initial longitudinal orientation to a mature transverse orientation. Panel A shows triads with variable orientations: longitudinal (double headed white arrow), oblique (white arrows), and correctly oriented transverse triads (small black arrows). The maturation of EC coupling apparatus closely follows the reorganization of the myofibrils: in panel B triads (black arrows) are already associate to I-A junction of well developed myofibrils, while are still missing where myofibrils are still not formed. In the transition area, forming triads are visible (small white arrows). Empty arrows in panels $A$ and B point at longitudinally oriented rows of mitochondria (see Figure 32 for more details).

established. Interestingly a similar mechanism has been already described in normally differentiating muscle fibers [75], and definitely would deserve a more detailed analysis. Unfortunately, the extremely limited availability of completely denervated biopsies did not allow us to study this phenomenon earlier during FES training. However at higher magnification we can also appreciate how the formation of nascent myofibrils seems to involve different steps such as an increase in the order of thin and thick filaments (empty arrows in Figure 29 D) and assemblage of primitive A bands (labeled in Figure 29 D) that also have been described as key steps of myofibril differentiation [76, 77].

In parallel to the restoration of myofibrils and the alignment of the cross striation, also the membrane systems involved in the EC coupling mechanism ( $\mathrm{T}$ tubules and SR) and the mitochondria become more numerous and find their specific location relative to the sarcomere' bands (Figure 30, Figure 31 and Figure 32).
Also in this case the reorganization of the EC coupling and mitochondrial apparatuses presents features that have been already described in muscle differentiation $[15,18,78]$. The development of $\mathrm{T}$ tubules in FES stimulated fibers involves the formation of multiple clusters of caveolae in the subsarcolemmal area (white arrows in Figure $30 \mathrm{~A}$ ) that are continuous with tortuous $\mathrm{T}$ tubules and labyrinthine structures located in the fiber interior (Figure 30, empty arrows). This network of maturing tubules becomes progressively better organized and more transversally oriented while it specifically associates to the I-A junction of mature myofibrils (Figure 30). In turn, the differentiation of a mature $\mathrm{T}$ tubule system is accompanied by the maturation of the SR network and by the formation of well differentiated triads, i.e. junction between the SR and the $\mathrm{T}$ tubules.

The final positioning of triads at their usual location in correspondence of the edges of the A band, characteristic of mammalian skeletal muscle, is 

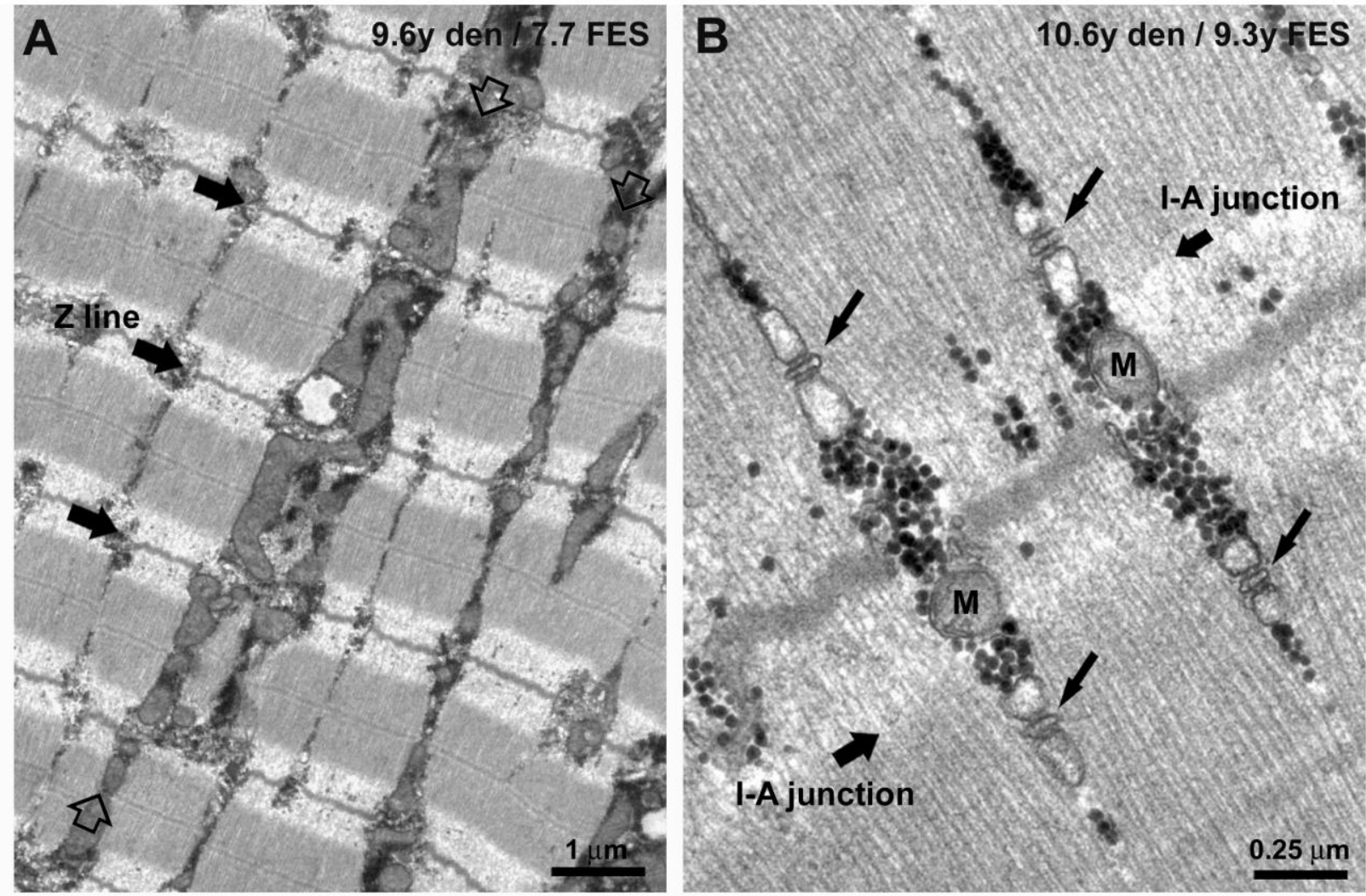

Fig 32 Progressive reorganization of the mitochondrial apparatus. The development of a mature mitochondrial apparatus present an initial stage in which mitochondria are found in clusters, primarily organized in longitudinally oriented rows, mostly located under the sarcolemma (not shown) and between myofibrils (panel $A$, empty arrows) followed by their final targeting next to triads (panel B, small back arrows), between the sarcomere A-I junction and the $Z$ line.

achieved by a gradual shift from an initial longitudinal to a mature transverse orientation (Figure $31 \mathrm{~A}$ ) This maturation of ECC coupling apparatus seems to closely follows the reorganization of the myofibrils: triads are in fact more frequent and better oriented in regions presenting well differentiated myofibrils than in those areas in which myofibrils are incomplete and/or still missing (Figure $31 \mathrm{~B}$ ). Finally, also the mitochondrial apparatus shows a progressive reorganization in recovering fibers: an initial stage in which mitochondria are found in clusters, primarily located in longitudinally oriented rows, is followed by their final targeting next to triads, i.e. between the edge of the $\mathrm{A}$ band and the $\mathrm{Z}$ Line (Figure 32 and Figure $33)$. These changes in the ultrastructural localization of mitochondria have been correlated to different developmental stages of muscle differentiation.

\section{DISCUSSION \\ 4.1 LONG-TERM DENERVATED HUMAN MUSCLE}

We showed that: 1 . the severe loss of mass in the thigh is the result of muscle atrophy and lipodystrophy
(Figure 21); 2. the overall disarrangement of the contractile apparatus that follows denervation is accompanied by nuclear changes, i.e. appearance of clumps of myonuclei (Figure 22) alternating with long stretches of anucleated sarcoplasm; 3. Approximately $1 \%$ of the myofibers stains positive by the antibody to MHCemb, indicating that they are fibers, which had regenerated during the last few weeks before biopsy (Figure 23). Embryonic myosin is expressed in myotubes and young myofibers and represents the soundest molecular marker of early myogenic events in both developing and adult muscles $[79,80]$; 4 . Electron microscopy analysis of DDM biopsies confirms the immunocytochemistry findings: double-layered basement membranes, delimiting myotubes and regenerated myofibers, respectively. During muscle regeneration, transient expression of laminin isoforms associated with early myogenesis was established. 5 . In addition to confirming the presence of regenerated myofibers, electron microscopy analysis shows not only the already described disorganization of both contractile apparatus, but also the disarrangement of the ECC apparatus (Fig. 3), thus explaining, in terms of 


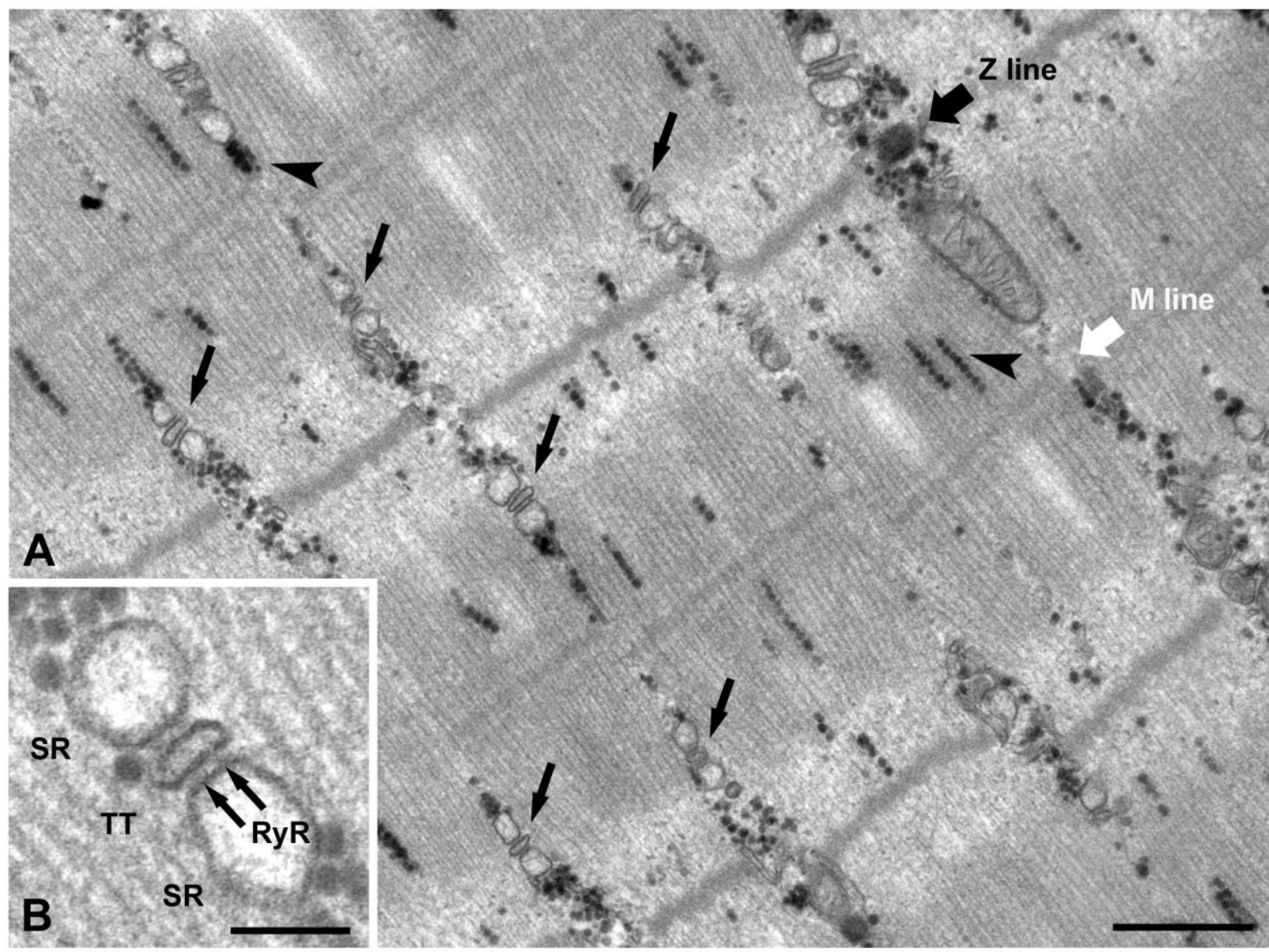

Fig 33 Effects of FES on the ultrastructure of long-term DDM. ECC apparatus re-associate to the A-I junction of the sarcomere (small black arrows) and the frequency of triads, or CRUs, is also restored in a way that almost every sarcomere has two triads on each side (small black arrows). B, triads now exhibit a normal profile (compare with Figure 11, showing junctions in normal human muscle): the triads in the long term DDM now contain RyRs (arrows), the Ca2+ release channel of the $S R$, two rows $n$ each side of the tubule, as in normal skeletal muscle junctions. A, scale bar: $0.5 \mu \mathrm{m} ; \mathrm{B}$, scale bar: $0.1 \mu \mathrm{m}$.

poor $\mathrm{Ca} 2+$ handling capacity, the low excitability of long-term denervated human muscle. Thus, the very high currents and extremely prolonged pulses needed to activate the atrophic DDM fibers [70] are due to the nearly complete and parallel disorganization of both ECC and contractile apparatuses.

These observations are novel and, to the knowledge of the authors, never before published, most likely because long-term effects of denervation in human muscles have attracted very limited attention. On the other hand, early denervation, as opposed to late, has been widely studied both in animal models and humans, since there is a general belief (mainly based on electrophysiological analyses) that all myofibers disappear within twelve months of SCI [81,82]. In rats, it has been shown that for the first 7 months after denervation, the myofibers exhibit a net loss of nuclear domains followed by nuclear groupings, a specific morphologic marker of long-term severe atrophy of rat muscle [83]. However, it has been also demonstrated in rodents that permanent lower motor neuron denervation is accompanied by a continuous production of new myofibers [41,83-85]. Indeed, we had been the first to show that regenerated myofibers are consistently present among atrophic myofibers even after one-year permanent denervation in both rat hemi-diaphragm [84] and leg muscles. Furthermore, in permanent absence of motor innervation, a completely regenerated muscle responds to repeated myotoxic injuries with muscle regeneration [41]. Embryonic myosin is expressed in myotubes and young myofibers and represents the soundest molecular marker of early myogenic events in both developing and adult muscle $[80,86]$. When re-innervation of regenerated myofibers occurs in adulthood, either slow or fast types of adult myosin may substitute the embryonic myosin 5-7 days 


\section{Muscle atrophy due to SCI can be reversed in complete absence of peripheral nerves}

European Journal Translational Myology - Basic Applied Myology 2012; 22 (4): 161-200

post-damage. On the other hand, if not re-innervated [85], the regenerated myofibers undergo atrophy and degeneration. In the present work we show that in FES muscles the regenerated myofibers, i.e., MHCembpositive fibers, are larger than those found in DDM muscles.

While it was common knowledge that denervation causes disarrangement of the myofibrils, not much was known until now about the effect of long-term denervation on the ECC apparatus. Takekura et al. [43] has shown that denervation of the tibialis anterior in rats causes several obvious structural changes in the disposition of the triads in relation to myofiber striation. As little as 2-3 weeks of postnatal denervation causes a striking increase in the longitudinal segments of the $\mathrm{T}$ tubule network and a remarkable change in the disposition of the triads, two characteristics commonly found in immature fibers and indeed present also in aneurally regenerated myofibers in adulthood [87]. In the present work, we have extended the studies on alterations of the ECC apparatus in human fibers denervated for longer periods of time: the junctions between $\mathrm{T}$ tubule and SR (i.e. triads) exhibit not only changes in their disposition but also a drastic decrease in their number. Furthermore, morphological features and the molecular composition of the triads are also affected: the SR and $\mathrm{T}$ tubule profiles in long-term denervated muscle are quite irregular, often dilated, and similar to junctions that have been described in developing or mutant muscle $[86,88]$. In addition, the junctions lose feet, i.e. the $\mathrm{Ca}^{2+}$ release channels of the SR, suggesting that, besides morphological alterations, there is also a functional impairment, i.e. the triad capacity to release $\mathrm{Ca}^{2+}$ is altered and possibly completely lost, providing a possible explanation for the needs of such long impulse duration and high intensity to be activated. As to the time course of changes, no final conclusions can be drawn on our results since we studied only 20 biopsies from 11 subjects (but only 5 time points, i.e., 1-, 3-, 4-, 8-, 20-year denervation). The trend suggests that the 1:4 ratio (one "defective" triad per $Z$ line over the expected four normal triads, which are present in innervated myofibers) is present from the earliest timepoint studied by electron microscopy (0.7-year) and lasts as long as studied (7.5-year), at least in those myofibers, which have sarcomeres. All together our observations point to the ECC system, as one of the key elements causing the very low excitability of the long-term denervated human muscle.

Though we have little data from humans that were in the transitional period ranging from early to late denervation, the results presented here suggest that the very low excitability of long-term denervated human muscle is the consequence of the segregation of the SR from the sarcolemma. The last conclusion is inferred from a pilot rat experiment in which animal groups were tested one-, two, four-, six-, and eight-month post-denervation. The transcutaneous current needed to activate denervated leg muscles increased with duration of post-denervation period. In vitro testing of the isolated muscles shown that the eight-month denervated rat muscle, which do not contract by direct electrostimulation, shortens when exposed to caffeine, a powerful releaser of $\mathrm{Ca}^{2+}$ from SR. This observation strongly complements the results of experiments designed to test the residual functional activity of muscle membranes (sarcolemma and SR) in the longterm denervated rat muscles. This appears to justify conclusions based on structural alterations observed in human long-term denervated muscles. Indeed, the RyR capacity, measured as 3H-ryanodine binding in purified SR membranes, was drastically altered and capable of significantly modifying the activity of $\mathrm{Ca}$ pumps, also present in these membranes. Vice versa, sarcolemma fractions, purified from the same muscles, showed a greater resistance to the effect of long-term denervation as indicated by both the Na-K ATPase activity and the presence of voltage-dependent $\mathrm{Ca}$ channels (measured as labeled DHPR binding), which were not statistically different with respect to the controls (unpublished observations).

\subsection{CLINICAL IMPORTANCE OF FES TRAINING IN SCI PATIENTS}

In DDM patients the improvements of muscle bulk, force and endurance are achieved through force and force-endurance exercises in 6-12 months training. Afterward, muscle are strengthened and endured enough to perform functional training, i.e., standing up exercises, and standing in parallel bars gait simulation by switching the tetanic electrostimulation of left/right quadriceps alternately. Effects of these treatments are not only a better cosmetic aspect, but also a better perfusion of the paralyzed legs. The continuous electrostimulation first changes the skin trophism (the skin is getting thicker and more resistant to electrical and mechanical stresses), and consequently eventual wound healing is faster. Thus, the risk of decubitus ulcer sharply decreases. Both biopsies and thigh CTscans show reduction of fat and connective tissue within and around the muscles. These findings indicate that degeneration of long-term denervated muscle is reversed by electrostimulation. Clinical observation of increased muscle mass and function (standing up exercises) correlate well with the measurable laboratory findings. High intensity stimulation training, i.e., with impulse parameters outside the current EU regulations, allows achieving within a relatively short time period (from 12 to 18 months) regeneration, hypertrophy and functional use of the denervated muscles. This high intensity stimulation program constitutes a risk only if the patients do not follow the instructions provided before he is enrolled in the stimulation program. Potential lesions are skin burns, if surface electrodes are not properly applied, and muscle over strain due to the absence of sensitivity (pain). 


\section{Muscle atrophy due to SCI can be reversed in complete absence of peripheral nerves}

European Journal Translational Myology - Basic Applied Myology 2012; 22 (4): 161-200

Though there are questions to be solved, we can say that risks for patients performing high intensity electrostimulation are low and that the FES training is effective if high currents are used. In contrast to prior insufficient treatments with exponential currents, high currents maintain trophism of denervated muscle for prolonged periods of time and even restore their structure and function after severe atrophy. High intensity stimulation allows patients to sit longer in the wheel chair because of the increased perfusion, the better trophism of the skin and the bigger muscle mass, which, reducing the risk of pressure sores, permits activities of daily living and thus a better social reintegration. Still there are many open problems, such as optimal time for onset, duration, intensity and methods of application of the FES training, but we firmly believe that in co-operation with international partners we will solve most of them in the time course of the European research project "RISE". Furthermore, we have the opportunity to study in permanent denervated human muscles both regenerative myogenesis and myofiber plasticity induced by electrical stimulation without any influence of the nerve. This will lead to new knowledge to establish better managements of elderly people and of patients with peripheral nerve lesions [71,89].

4.3 IMPORTANCE OF FES ON DENERVATED SKELETAL MUSCLE

The main finding of this work is that the degeneration of muscle fibers that follows long-term denervation can be reversed using the FES protocol developed in the last years $[68,70,89]$. More than $50 \%$ of myofibers have diameter smaller than $10 \mu \mathrm{m}$ in DDM biopsies, while the FES trained subjects have more that $50 \%$ of myofibers larger than $30 \mu \mathrm{m}$ (Figure 28). Abnormalities up to the complete absence of organized myofibrillar structures are the obvious consequences of denervation and absence of contractility. However, DDM human muscle can still respond with single twitches if very high electrical stimulation is applied $[68,70,89]$. The most important finding of our work is that the FES protocol used during training of our patients reverses the massive structural alteration of both contractile and ECC apparatuses in human DDM fibers. Electrostimulation effectively elicited sustained muscle contractions and CT-scans measurements taken during the first few years of functional electrical stimulation revealed an increase in the tight muscle cross-sectional area and tissue density.

Electron microscopy analysis shows a striking positive structural effect of FES training on DDM fibers (compare Figure 26 and Figure 33). In the clinical treatment of muscle paralysis, the poor recovery of muscle tissue which has been denervated for prolonged periods of time has been a longstanding problem. However, the results presented in this paper clearly indicate that long-term denervated human muscles (up to 2 years of complete denervation) can be effectively rescued if appropriately stimulated. This recovery can occur in complete absence of nerve endings under the influence of muscle activity induced by the FES training. We have also shown that these FES devices specifically designed to treat patients with no peripheral nerves in the lower extremities, are extremely effective in promoting structural recovery of degenerated muscles both qualitatively and quantitatively (Figure 33 and Table VI). These morphological findings provide the structural basis for the functional recovery in SCI patients presented in Kern et al., 1999 and Modlin et al., 2005 [68,71].

Interestingly, the mechanisms by which the ultrastructure of myofibrils is rescued and the EC coupling and metabolic machinery is rearranged closely mimics the normal process of myofiber differentiation both in the apparent spread of events from the periphery to the center of the fiber (Figure $29 \mathrm{C}$ ) and in various intermediate stages that lead to final organization of different apparatuses. In short: a) the formation of nascent myofibrils involves initial alignment of thin and thick filaments and assemblage of primitive A bands (Figure 29 D); b) the development of T tubules, and SR membrane is achieved by a gradual shift from an initial longitudinal to a mature transverse orientation (Figures 29 and 30); c) the maturation of the metabolic apparatus present an initial stage of longitudinal grouping of mitochondria followed by the final association to the I band of mature sarcomeres (Figure 32) All these features has been already described as key intermediate steps in muscle fiber differentiation $[15,18,75,76,77,78]$, but what is interesting in this is the fact that the differentiation program is being played in complete absence of nerve endings. Although there is an increased appreciation for the importance of intrinsic genetic programming in the differentiation of muscle fiber, it is still generally accepted that innervation is necessary for the normal maturation and differentiation of myofibers. However, there are some data in literature indicating that innervation is not needed for the differentiation of primary myotubes into mature muscle fibers [90,91], and our results show that a structural differentiation may be achieved without the nerve and under the influence of induced muscle activity. At this stage, we have no indication on contraction kinetics and metabolic pathways of the recovering fibers in our patients and we cannot exclude the importance of the nerve for some aspect of the fiber maturation. In addition, it is difficult to determine the differential roles of the electrical stimulation itself and of the induced muscle activity in the different aspects of the regeneration process.

The apparently related increase in differentiated fibers and decrease in clearly atrophic ones in stimulated vs. control muscles (Table V and VI), and the presence of fibers that show a regenerating periphery together with an atrophic core, would argue in favor of a 


\section{Muscle atrophy due to SCI can be reversed in complete absence of peripheral nerves}

European Journal Translational Myology - Basic Applied Myology 2012; 22 (4): 161-200

regeneration process mediated by the re-activation of the myogenic program in the severely atrophic fibers. We have previously shown that even severely atrophic fibers do contain nuclei [72]. In addition, there is evidence in literature indicating that the number of nuclei in long-term denervated muscle is unvaried [92] and that those nuclei do not show DNA breaks [93]. We could speculate that these nuclei could be in quiescent-like state in long-term denervated fibers and that could intervene and support the myogenic program that allows muscle fiber recovery during the FES training. Obviously, we can not exclude a role of satellite cells in this process. However, due to limitations in the use of human biopsies, we could not determine whether, and in which measure, satellite cells are recruited. There are reports in the literature indicating that, while short-term denervation induces satellite cells to enter the cell cycle, long-term denervation causes a drastic reduction in their number and activity, and even apoptosis [94-97]. We also know from our previous studies that regenerating myotubes form spontaneously, but at an extremely low rate, even several years after an initial injury [72]. These observations altogether suggest that, while recruitment of satellite cells could somehow contribute to the muscle recovery, this mechanism is probably not the primary event in our specimens.

Independent of which molecular process allows muscle to be rescued, we believe that the results of our work may be important both for the rehabilitation of SCI patients and, more importantly, for the future possibility of re-creating a connection between nerve endings and muscle fibers. FES stimulation has been proven to be an effective method used to retard muscle atrophy and improve recovery after reinnervation $[69,71]$. Sophisticated FES devices have been developed for restoring function in the upper and lower extremities, the bladder and bowel, and the respiratory system of SCI patients [70]. However, there are SCI cases, such as those affected by flaccid paralysis, in which the musculature is not treated with FES rehabilitation therapy. This is because conventional FES apparatuses are designed for direct stimulation of peripheral nerves that need small currents to be depolarized, and are not effective in patients that have lost their peripheral nerves, and, therefore, require higher currents for the direct depolarization of the muscle fibers. Lack of muscle treatment generates, as a secondary problem, a long series of alterations to tissues other than muscle, such as bones (osteoporosis), skin (pressure sores, decubital ulcers), etc., that are a direct consequence of inactivity and poor blood supply to the denervated areas. These complications represent an extremely serious problem for the general health of the injured individuals, who usually have a shorter than normal life span. The stimulator and electrodes developed for our studies offers the following advances: a) can deliver of larger currents that can directly elicit muscle contractions, even after long periods of denervation; and b) can be used to recover and/or maintain muscle tissue in paraplegic patients. Regenerating (and/or better maintaining) muscle fibers that have lost their motor neurons, is helping to avoid some of the secondary problems since daily training promotes blood flow to the denervated areas of the body.

Beside the more immediate, and more obvious, applications of this FES protocol for the rehabilitation and general health of the patients, these findings could also have significant implications in basic research and for the future possibility of reconnecting the nerve to the target muscle fibers. One of the possible causes for the difficulties in re-creating a connection between nerve and muscle can be found in the rapid decline of denervated fibers that could indeed interfere with reinnervation events [39]. The structural results presented in this paper support this hypothesis: the TEM analysis of biopsies from the SCI patients shows that after only a year of denervation, human muscle fibers are already severely compromised (Figure $25 \mathrm{~A}$ and Table V, patients SCI 1 and 2). It is possible to speculate that a FES trained muscle fiber (Figure 33), may represent a better target for a re-growing nerve than a severely atrophic and degenerated fiber surrounded by collagen and fat (Figure $25 \mathrm{~B}$ ).

Could the recovery and/or maintenance of muscle facilitate nerve re-growth and reinnervation events? Could a functional muscle apparatus represent a better target for a re-growing nerve? These are issues that still need to be addressed more in detail since the existing literature reports conflicting findings on the effects of muscle activity and/or electrical stimulation on nerve sprouting and possible reinnervation events [98-104], and does not address the question if whether or not the degeneration of muscle tissue that follows denervation may, in the long-term, represent an additional factor interfering with reinnervation.

Abbreviations
CICR, calcium induced calcium release.
CNS, central nervous system.
CRU, calcium release unit.
DDM, denervated and degenerated muscle.
DHPR, dihydropyridine receptor.
ECC, excitation-contraction coupling.
EM, electron microscopy.
FES, functional electrical stimulation.
FNS, functional neuromuscular stimulation.
H\&E, hematoxilin and eosin.
RyR, ryanodine receptor.
SCI, spinal cord injury.
SM, surface membrane.
SR, sarcoplasmic reticulum.
TEM, transmission electron microscopy.
T-tubule transverse tubule.




\section{Muscle atrophy due to SCI can be reversed in complete absence of peripheral nerves}

European Journal Translational Myology - Basic Applied Myology 2012; 22 (4): 161-200

\section{Acknowledgements}

This work was carried out at the Center of Science on Aging, at the Foundation of the Gabriele D'Annunzio University.

I am most grateful to my tutor, Feliciano Protasi. First of all, I want to thank him for the confidence he gave me as a novice research by starting a new research area, the biology, and for attracting and introducing me to the biological field of muscle. I wish to express my gratitude for always being available for valuable help, guidance and support during this project. Most of all, reminding me about the importance of keeping the focus towards a precise objective and giving very valuable advices.

Second, I thank Prof. Ugo Carraro, primly for giving me the big opportunity to work with denervated human muscle, for his great enthusiasm and also for access to his vast knowledge about previous research on muscle denervation. I also thank Helmut Kern and Winfried Mayr, and all the staff working in their laboratories, which with many years of dedicated work allowed the collection of these samples and the acquisition of these data.

I thanks Giorgio Fanò for the lab spaces and financial support that have allowed us to start working in the University of Chieti and also for take me home with Stefania Fulle, when I'm nostalgic.

Dante Tatone for his technical help in setting up our new laboratory in the CeSI building. and for providing excellent working facilities for the research.

I owe my gratitude to my colleague and friend in the area of microscopy, Cecilia and also Cristina for share with me their knowledge about this field. I want to thank also Marco for his technical helps with the computer!

I want to thank warmly my friends, Claudia, Giusi, my cousin Annik, and Laura (Ce.S.I.) for gave me relaxing and joyful moment when I needed a break from the research.

Finally, and most importantly, I would like to thank my parents, "il babbo Nivo" e "la Mamma Alessandra" for their unflagging dedication, love and absolute confidence on me; to my brother, Emanuele, just because is the best brother that I have... the only one, but is the same.

I'd like to dedicate this work to my grandmother, "la nonna Anna". "I remember the day of my graduation: you was touched and now that memory touched me. I'm sure you would come in Chieti and you would appreciate my work. I love u so much..."

"Imagination is more important that knowledge; knowledge is limited while imagination embraces the entire world."

Albert Einstein

\section{Corresponding Author}

Simona Boncompagni, CeSI - Center for Research on Aging \& DNI - Dept Neuroscience and Imaging, University G. d'Annunzio of Chieti, Italy.

E-mail: s.boncompagni@unich.it

\section{References}

[1] Ishikawa H. Fine structure of skeletal muscle. Cell Muscle Motil 1983; 4: 1-84.

[2]. Peachey LD. Excitation-contraction coupling: the link between the surface and the interior of a muscle cell. J Exp Biol 1985; 115: 91-98.

[3] Mauro A. Satellite cell of skeletal muscle fibers. J Biophys Biochem Cytol 1961; 9: 493-495.

[4] Loeb GE, Gans C. Electromyography for experimentalists. 1986 .

[5] Bagshaw CR. Outline Studies of Biology: Muscle Contraction. London: Chapman and Hall; 1982. p. 22.

[6] Eisenberg BR. Adaptability of ultrastructure in the mammalian muscle. J Exp Biol 1985; 115: 55-68.

[7] Franzini-Armstrong C, Porter KR,. The Z Disc of Skeletal Muscle Fibrils. Z Zellforsch Mikrosk Anat 1964; 61: 661-672.

[8] Huxley H, Hanson J. Changes in the crossstriations of muscle during contraction and stretch and their structural interpretation. Nature 1954; 173: 973-976.

[9] Huxley AF, Niedergerke R. Structural changes in muscle during contraction; interference microscopy of living muscle fibres. Nature 1954; 173: 971-973.

[10] Franzini-Armstrong C, Porter KR. Sarcolemmal Invaginations Constituting The $\mathrm{T}$ System In Fish Muscle Fibers. J Cell Biol 1964; 22: 675696.

[11] Porter KR, Franzini-Armstrong C. The Sarcoplasmic Reticulum. Sci Am 1965; 212: 72-81.

[12] Flucher BE. Structural analysis of muscle development: transverse tubules, sarcoplasmic reticulum, and the triad. Dev Biol 1992; 154: 245-260.

[13] Brum G, Fitts R, Pizarro G, Rios E. Voltage sensors of the frog skeletal muscle membrane require calcium to function in excitationcontraction coupling. J Physiol 1988; 398: 475505.

[14] Stokes DL, Wagenknecht T. Calcium transport across the sarcoplasmic reticulum: structure and function of $\mathrm{Ca} 2+-\mathrm{ATPase}$ and the ryanodine receptor. Eur J Biochem 2000; 267: 5274-5279.

[15] Franzini-Armstrong C, Jorgensen AO. Structure and development of E-C coupling units in 


\section{Muscle atrophy due to SCI can be reversed in complete absence of peripheral nerves}

European Journal Translational Myology - Basic Applied Myology 2012; 22 (4): 161-200

skeletal muscle. Annu Rev Physiol 1994; 56: 509-534.

[16] Flucher BE, Franzini-Armstrong C. Formation of junctions involved in excitation-contraction coupling in skeletal and cardiac muscle. Proc Natl Acad Sci U S A 1996; 93: 8101-8106.

[17] Fabiato A. Calcium-induced release of calcium from the cardiac sarcoplasmic reticulum. Am J Physiol 1983; 245: C1-14.

[18] Flucher BE, Takekura H, Franzini-Armstrong C. Development of the excitation-contraction coupling apparatus in skeletal muscle: association of sarcoplasmic reticulum and transverse tubules with myofibrils. Dev Biol 1993; 160: 135-147.

[19] Sommer JR. Comparative anatomy: in praise of a powerful approach to elucidate mechanisms translating cardiac excitation into purposeful contraction. J Mol Cell Cardiol 1995; 27: 19-35.

[20] Protasi F. Structural interaction between RYRs and DHPRs in calcium release units of cardiac and skeletal muscle cells. Front Biosci 2002; 7 : d650-658.

[21] Franzini-Armstrong C. Studies of the triad. J Cell Biol 1970; 47: 488-499.

[22] Lai FA, Anderson K, Rousseau E, Liu QY, Meissner G. Evidence for a $\mathrm{Ca} 2+$ channel within the ryanodine receptor complex from cardiac sarcoplasmic reticulum. Biochem Biophys Res Commun 1988; 151: 441-449.

[23] Tanabe T, Takeshima H, Mikami A, Flockerzi V, Takahashi H, Kangawa K, Kojima M, Matsuo H, Hirose T, Numa S. Primary structure of the receptor for calcium channel blockers from skeletal muscle. Nature 1987; 328: 313318.

[24] Jorgensen AO, Shen AC, Arnold W, Leung AT, Campbell KP. Subcellular distribution of the 1,4-dihydropyridine receptor in rabbit skeletal muscle in situ: an immunofluorescence and immunocolloidal gold-labeling study. J Cell Biol 1989; 109: 135-147.

[25] Carl SL, Felix K, Caswell AH, Brandt NR, Ball WJ, Jr., Vaghy PL, Meissner G, Ferguson DG. Immunolocalization of sarcolemmal dihydropyridine receptor and sarcoplasmic reticular triadin and ryanodine receptor in rabbit ventricle and atrium. J Cell Biol 1995; 129: 672-682.

[26] Block BA, Imagawa T, Campbell KP, FranziniArmstrong C. Structural evidence for direct interaction between the molecular components of the transverse tubule/sarcoplasmic reticulum junction in skeletal muscle. J Cell Biol 19881; 07: 2587-2600.
[27] Engel AG, The neuromuscolar junction. Myology (third edition). I: 325-372.

[28] Grinnell AD, Trophic Interaction between Nerve and Muscle. Myology (third edition). I: 303-332.

[29] Grinnell AD. Dynamics of nerve-muscle interaction in developing and mature neuromuscular junctions. Physiol Rev 1995; 75: 789-834.

[30] Sanes JR, Lichtman JW. Development of the vertebrate neuromuscular junction. Annu Rev Neurosci 1999; 22: 389-442.

[31] Pette D, Vrbova G. Neural control of phenotypic expression in mammalian muscle fibers. Muscle Nerve 1985; 8: 676-689.

[32] Buller AJ, Eccles JC, Eccles RM. Interactions between motoneurones and muscles in respect of the characteristic speeds of their responses. J Physiol 1960 ; 150: 417-439.

[33] Waters RL, Adkins RH, Yakura JS. Definition of complete spinal cord injury. Paraplegia 1991; 29: 573-581.

[34] Ditunno JF, Little JW, Tessler A, Burns AS,. Spinal shock revisited: a four-phase model. Spinal Cord 2004; 42: 383-395.

[35] Pellegrino C, Franzini-Armstrong C. Recent contributions of electron microscopy to the study of normal and pathological muscle. J Cell Biol 1969; 7: 139-226.

[36] Engel AG, Stonnington HH. Trophic functions of the neuron. II. Denervation and regulation of muscle. Morphological effects of denervation of muscle. A quantitative ultrastructural study. Ann N Y Acad Sci 1974; 228: 68-88.

[37] Lu DX, Huang SK, Carlson BM. Electron microscopic study of long-term denervated rat skeletal muscle. Anat Rec 1997; 248: 355-365.

[38] Katz B, Miledi R. The Development Of Acetylcholine Sensitivity In Nerve-Free Segments Of Skeletal Muscle. J Physiol 1964; 170: 389-396.

[39] Card DJ. Denervation: sequence of neuromuscular degenerative changes in rats and the effect of stimulation. Exp Neurol 1977; 54: 251-265.

[40] Pellegrino C, Franzini-Armstrong C. An electron microscopy study of denervation atrophy in red and white skeletal muscle fibers. J Cell Biol 1963; 17: 327-349.

[41] Viguie CA, Lu DX, Huang SK, Rengen H, Carlson BM. Quantitative study of the effects of long-term denervation on the extensor digitorum longus muscle of the rat. Anat Rec 1997; 248: 346-54. 


\section{Muscle atrophy due to SCI can be reversed in complete absence of peripheral nerves}

European Journal Translational Myology - Basic Applied Myology 2012; 22 (4): 161-200

[42] Carlson BM. Skeletal muscle regeneration during aging and after long-term denervation. Tsitologiia 1997; 39: 965-968.

[43] Takekura H, Kasuga N. Differential response of the membrane systems involved in excitationcontraction coupling to early and later postnatal denervation in rat skeletal muscle. J Muscle Res Cell Motil 1999; 20: 279-289.

[44] Takekura H, Kasuga N, Kitada K, Yoshioka T. Morphological changes in the triads and sarcoplasmic reticulum of rat slow and fast muscle fibres following denervation and immobilization. J Muscle Res Cell Motil 1996; 17: 391-400.

[45] Takekura H, Tamaki H, Nishizawa T, Kasuga $\mathrm{N}, 2003$. Plasticity of the transverse tubules following denervation and subsequent reinnervation in rat slow and fast muscle fibres. J Muscle Res Cell Motil 24: 439-451.

[46] Borisov AB, Dedkov EI, Carlson BM. Interrelations of myogenic response, progressive atrophy of muscle fibers, and cell death in denervated skeletal muscle. Anat Rec 2001; 264: 203-218.

[47] Borisov AB, Carlson BM. Cell death in denervated skeletal muscle is distinct from classical apoptosis. Anat Rec 2000; 258: 305318.

[48] Guth L, Kemerer VF, Samaras TA, Warnick JE, Albuquerque EX, 1. The roles of disuse and loss of neurotrophic function in denervation atrophy of skeletal muscle. Exp Neurol 198; 73: 20-36.

[49] Lomo T, Rosenthal J. Control of ACh sensitivity by muscle activity in the rat. J Physiol 1972; 221: 493-513.

[50] Lomo T. [Denervation and reinnervation of muscle]. Tidsskr Nor Laegeforen 1975; 95: 354356.

[51] Jones R, Vrbova G. Two factors responsible for the development of denervation hypersensitivity. J Physiol 1974; 236: 517-538.

[52] Cangiano A, Fried JA. The production of denervation-like changes in rat muscle by colchicine, without interference with axonal transport or muscle activity. J Physiol 1977; 265: 63-84.

[53] Hughes JT, Brownell B. Ultrastructure of muscle in Werdnig-Hoffmann disease. J Neurol Sci 1969; 8: 361-379.

[54] Belal A, Jr. Structure of human muscle in facial paralysis. J Laryngol Otol 1982; 96: 325-334.

[55] Schwab ME. Increasing plasticity and functional recovery of the lesioned spinal cord. Prog Brain Res 2002; 137: 351-359.
[56] Bunge MB, Pearse DD. Transplantation strategies to promote repair of the injured spinal cord. J Rehabil Res Dev 2003; 40: 55-62.

[57] Xiang S, Pan W, Kastin AJ. Strategies to create a regenerating environment for the injured spinal cord. Curr Pharm Des 2005; 11: $1267-$ 1277.

[58] Fu SY, Gordon T. Contributing factors to poor functional recovery after delayed nerve repair: prolonged axotomy. J Neurosci 1995; 15: 38763885 .

[59] Campos L, Ambron RT, Martin JH. Bridge over troubled waters. Neuroreport 2004; 15: 26912694.

[60] Kobayashi J, Mackinnon SE, Watanabe O, Ball DJ, Gu XM, Hunter DA, Kuzon WM, Jr. The effect of duration of muscle denervation on functional recovery in the rat model. Muscle Nerve 1997; 20: 858-866.

[61] Aydin MA, Mackinnon SE, Gu XM, Kobayashi J, Kuzon WM, Jr.. Force deficits in skeletal muscle after delayed reinnervation. Plast Reconstr Surg 2004; 113: 1712-1718.

[62] Lomo T, Slater CR. Proceedings: Induction of ACh sensitivity at new neuromuscular junctions. J Physiol 1976; 258: 107P-108P.

[63] Nemoto K, Williams HB, Nemoto K, Lough J, Chiu RC. The effects of electrical stimulation on denervated muscle using implantable electrodes. J Reconstr Microsurg 19761988; 4: 251-255, 257.

[64] Kanaya F, Tajima T. Effect of electrostimulation on denervated muscle. Clin Orthop Relat Res. 1992 Oct;(283):296-301.

[65] Williams HB. The value of continuous electrical muscle stimulation using a completely implantable system in the preservation of muscle function following motor nerve injury and repair: an experimental study. Microsurgery 1996; 17: 589-596.

[66] Bateman JE. Athletic injuries about the shoulder in throwing and body-contact sports. Clin Orthop 1962; 23: 75-83.

[67] Sunderland S,. Advances in diagnosis and treatment of root and peripheral nerve injury. Adv Neurol 1979; 22: 271-305.

[68] Kern H, Hofer C, Strohhofer M, Mayr W, Richter W, Stohr H. Standing up with denervated muscles in humans using functional electrical stimulation. Artif Organs 1999; 23: 447-452.

[69] Mayr W, Bijak M, Rafolt D, Sauermann S, Unger E, Lanmuller $H$. Basic design and construction of the Vienna FES implants: existing solutions and prospects for new 


\section{Muscle atrophy due to SCI can be reversed in complete absence of peripheral nerves}

European Journal Translational Myology - Basic Applied Myology 2012; 22 (4): 161-200

generations of implants. Med Eng Phys 2001; 23: 53-60.

[70] Hofer C, Mayr W, Stohr H, Unger E, Kern H. A stimulator for functional activation of denervated muscles. Artif Organs 2002; 26: 276-279.

[71] Modlin M, Forstner C, Hofer C, Mayr W, Richter W, Carraro U, Protasi F, Kern H. Electrical stimulation of denervated muscles: first results of a clinical study. Artif Organs 2005; 29: 203-206.

[72] Kern H, Boncompagni S, Rossini K, Mayr W, Fano G, Zanin ME, Podhorska-Okolow M, Protasi F, Carraro U. Long-term denervation in humans causes degeneration of both contractile and excitation-contraction coupling apparatus, which is reversible by functional electrical stimulation (FES): a role for myofiber regeneration? J Neuropathol Exp Neurol 2004; 63: 919-931.

[73] Kern H, Hofer C, Modlin M, Forstner C, Mayr W, Richter W. Functional electrical stimulation (FES) of long-term denervated muscles in humans: clinical observations and laboratory findings. Basic Appl Myol 2002; 12: 291-297.

[74] Engel AG, Banker BQ, Ultrastructural changes in diseased muscle. In Egel A. G. \& FranziniArmstrong.(eds). Myology (third edition). 1: 749-887.

[75] Morkin E. Postnatal muscle fiber assembly: localization of newly synthesized myofibrillar proteins. Science 1970; 167: 1499-1501.

[76] Devlin RB, Emerson CP, Jr. Coordinate regulation of contractile protein synthesis during myoblast differentiation. Cell 1978; 13: 599-611.

[77] Epstein HF, Fischman DA. Molecular analysis of protein assembly in muscle development. Science 1991; 251: 1039-1044.

[78] Franzini-Armstrong C. Simultaneous maturation of transverse tubules and sarcoplasmic reticulum during muscle differentiation in the mouse. Dev Biol 1991; 146: 353-363.

[79] Whalen RG, Sell SM, Butler-Browne GS, Schwartz K, Bouveret P, Pinset-Harstom I. Three myosin heavy-chain isozymes appear sequentially in rat muscle development. Nature 1981; 292: 805-809.

[80] Carraro U, Dalla Libera L, Catani C. Myosin light and heavy chains in muscle regenerating in absence of the nerve: transient appearance of the embryonic light chain. Exp Neurol 1983; 79: 106-117.
[81] Gutmann E. Denervation and disuse atrophy in crosstriated muscle. Rev Can Biol 1962; 21: 353-365.

[82] Drachman DB, Murphy SR, Nigam MP, Hills JR. "Myopathic" changes in chronically denervated muscle. Arch Neurol 1967; 16: 1424.

[83] Carraro U, Morale D, Mussini I, Lucke S, Cantini M, Betto R, Catani C, Dalla Libera L, Danieli Betto D, Noventa D. Chronic denervation of rat hemidiaphragm: maintenance of fiber heterogeneity with associated increasing uniformity of myosin isoforms. J Cell Biol 1985 100: 161-74.

[84] Mussini I, Favaro G, Carraro U. Maturation, dystrophic changes and the continuous production of fibers in skeletal muscle regenerating in the absence of nerve. J Neuropathol Exp Neurol 1987; 46: 315-31.

[85] Carraro U, Catani C, Dalla Libera L. Myosin light and heavy chains in rat gastrocnemius and diaphragm muscles after chronic denervation or reinnervation. Exp Neurol 1981; 72: 401-412.

[86] Protasi F, Takekura H, Wang Y, Chen SR, Meissner G, Allen PD, Franzini-Armstrong C. RYR1 and RYR3 have different roles in the assembly of calcium release units of skeletal muscle. Biophys J 2000; 79: 2494-2508.

[87] Carraro U, Catani C, Biral D. Selective maintenance of neurotrophically regulated proteins in denervated rat diaphragm. Exp Neurol 1979; 63: 468-475.

[88] Takekura H, Nishi M, Noda T, Takeshima H, Franzini-Armstrong C. Abnormal junctions between surface membrane and sarcoplasmic reticulum in skeletal muscle with a mutation targeted to the ryanodine receptor. Proc Natl Acad Sci U S A 1995; 92: 3381-3385.

[89] Kern H, Hofer C, Modlin M, Forstner C, Raschka-Hogler D, Mayr W, Stohr H. Denervated muscles in humans: limitations and problems of currently used functional electrical stimulation training protocols. Artif Organs 2002; 26: 216-218.

[90] Condon K, Silberstein L, Blau HM, Thompson WJ. Differentiation of fiber types in aneural musculature of the prenatal rat hindlimb. Dev Biol 1990; 138: 275-295.

[91] Hoh JF. Myogenic regulation of mammalian skeletal muscle fibres. News Physiol Sci 1991; 6: 1-6.

[92] Wada KI, Takahashi H, Katsuta S, Soya H. No decrease in myonuclear number after long-term denervation in mature mice. Am J Physiol Cell Physiol 2002; 283: C484-488. 


\section{Muscle atrophy due to SCI can be reversed in complete absence of peripheral nerves}

European Journal Translational Myology - Basic Applied Myology 2012; 22 (4): 161-200

[93] Rodrigues Ade C, Schmalbruch H. Satellite cells and myonuclei in long-term denervated rat muscles. Anat Rec 1995; 243: 430-437.

[94] Murray MA, Robbins N. Cell proliferation in denervated muscle: identity and origin of dividing cells. Neuroscience 1982; 7: 18231833.

[95] Kuschel R, Yablonka-Reuveni Z, Bornemann A. Satellite cells on isolated myofibers from normal and denervated adult rat muscle. J Histochem Cytochem 1999; 47: 1375-1384.

[96] Dedkov EI, Kostrominova TY, Borisov AB, Carlson BM. Reparative myogenesis in longterm denervated skeletal muscles of adult rats results in a reduction of the satellite cell population. Anat Rec 2001; 263: 139-154.

[97] Jejurikar SS, Marcelo CL, Kuzon WM, Jr. Skeletal muscle denervation increases satellite cell susceptibility to apoptosis. Plast Reconstr Surg 2002; 110: 160-168.

[98] Brown MC, Holland RL. A central role for denervated tissues in causing nerve sprouting. Nature 1979; 282: 724-726.

[99] Al-Majed AA, Neumann CM, Brushart TM, Gordon T. Brief electrical stimulation promotes the speed and accuracy of motor axonal regeneration. J Neurosci 2000; 20: 2602-1608.

[100] Rozman J, Zorko B, Seliskar A. Regeneration of the radial nerve in a dog influenced by electrical stimulation. Pflugers Arch 2000; 439: R184-186.

[101] Tam SL, Archibald V, Jassar B, Tyreman N, Gordon T. Increased neuromuscular activity reduces sprouting in partially denervated muscles. J Neurosci 2001; 21: 654-667.

[102] Brushart TM, Hoffman PN, Royall RM, Murinson BB, Witzel C, Gordon T. Electrical stimulation promotes motoneuron regeneration without increasing its speed or conditioning the neuron. J Neurosci 2002; 22: 6631-6638.

[103] Zealear DL, Rodriguez RJ, Kenny T, Billante MJ, Cho Y, Billante CR, Garren KC. Electrical stimulation of a denervated muscle promotes selective reinnervation by native over foreign motoneurons. J Neurophysiol 2002; 87: 2195-9.

[104] Mendonca AC, Barbieri CH, Mazzer N. Directly applied low intensity direct electric current enhances peripheral nerve regeneration in rats. J Neurosci Methods 2003; 129: 183-190. 